\title{
Harmonic maps between singular spaces I
}

\author{
Georgios Daskalopoulos and Chikako Mese
}

\begin{abstract}
We discuss regularity questions for harmonic maps from a $n$-dimensional Riemannian polyhedral complex $X$ to a non-positively curved metric space. The main theorems assert, assuming Lipschitz regularity of the metric on the domain complex, that such maps are locally Hölder continuous with explicit bounds of the Hölder constant and exponent on the energy of the map and the geometry of the domain and locally Lipschitz continuous away from the $(n-2)$ - skeleton of the complex. Moreover, if $x$ is a point on the $k$-skeleton $(k \leq n-2)$ we give explicit dependence of the Hölder exponent at a point near $x$ on the combinatorial and geometric information of the link of $x$ in $X$ and the link of the $k$-dimensional skeleton in $X$ at $x$.
\end{abstract}

\section{Introduction}

The seminal work of Gromov and Schoen [9] extends the study of harmonic maps between smooth manifolds to the case when the target is a Riemannian simplicial complex of non-positive curvature. The theory of harmonic maps into singular spaces was expanded substantially by the work of Korevaar and Schoen [11, 12] and Jost [10] where they consider targets that are arbitrary metric spaces of non-positive curvature. (Such spaces are called NPC or CAT(0) if they are simply connected.) One important motivation for considering singular spaces in the theory of harmonic maps is in studying group representations. The main application of the Gromov-Schoen theory is to establish a certain case of non-Archemedean superrigidity complementing Corlette's Archemedean superrigidity for lattices in groups of real rank 1 [3].

The next step in the generalization of the harmonic map theory is to replace smooth domains by singular ones. This problem is also motivated by superrigidity, in this case when the domain group is non-Archemedean. The consideration of a Riemannian simplicial complex as the domain space for harmonic maps seems to have been initiated by Chen [2]. Subsequently, this theory was further elaborated by Eells and Fuglede [7] and Fuglede [8]. In particular, they show Hölder continuity for harmonic maps under an appropriate smoothness assumption for the metric on each simplex. 
The development of the harmonic map theory from a Riemannian complex is important in the study of non-Archedemean lattices. Considering a two-dimensional domains, Daskalopoulos and Mese [4-6] establish fixed point and rigidity theorems of harmonic maps from certain flat two-complexes. The key issue in the techniques introduced in these papers is to prove regularity theorems strong enough to be able to apply differential geometric methods.

Recall that the main idea of [9] is also to show that harmonic maps are regular enough so that Bochner methods could be used in the setting of singular targets. In particular, the fundamental regularity result of $[9,11]$ is that harmonic maps from a smooth Riemannian domain into an NPC target are locally Lipschitz continuous. As noted in [2], this statement no longer holds when we replace the domain by a polyhedral space. On the other hand, we have found in $[5,6]$ that modulus of continuity better than Hölder is crucial in applications. This necessitates stronger regularity results than Hölder.

This paper is meant to be the state of the art in the regularity theory of harmonic maps from Riemannian cell complexes to non-positively curved metric spaces (cf. Section 2 for precise definitions). Our first theorem concerns Hölder continuity of harmonic maps. This is a generalization of the result of [7] for Lipschitz metrics.

Theorem 1.1 (cf. Theorem 4.3). Let $B(r)$ be a ball or radius $r$ around a point in an admissible complex $X$ endowed with a Lipschitz Riemannian metric $g$ and $(Y, d)$ an NPC space. If $f:(B(r), g) \rightarrow(Y, d)$ is a harmonic map, then there exist $C$ and $\gamma>0$ so that

$$
d(f(x), f(y)) \leq C|x-y|^{\gamma} \forall x, y \in B(\varrho r)
$$

Here, $C$ and $\gamma$ only depend on the total energy $E^{f}$ of the map $f,(B(r), g)$ and $\varrho \in(0,1)$.

Note that our approach to Hölder continuity follows the one in $[2,9]$ and is completely different from the one in [7, 8]. In our case, a variant of the Gromov-Schoen monotonicity formula allows us to obtain energy decay estimates which in turns imply Hölder continuity by an adaptation of an argument due to Morrey. The technical difficulty is that we make no assumption that the boundary of each simplex is totally geodesic as it is implicitly assumed in [2]. Our method also differs from the one in [9] due to the fact that for singular domains the monotonicity formula does not hold 
for large balls (cf. Remark 3.3 of [4]). The main technical hurdle is to obtain energy decay estimates with uniform radius and this is handled in Section 4.

Our main theorem concerns better regularity of harmonic maps. More precisely, we show that harmonic maps are Lipschitz continuous away from the codimension 2 skeleton $X^{(n-2)}$ of $X$. For points that lie on the lower dimensional skeleta, we also give an estimate of the Hölder exponent of the harmonic map in terms of the first eigenvalue of the link of the normal stratum of the skeleton. More precisely, let $x \in X^{(k)}-X^{(k-1)}$ and let $N=$ $N(x)$ denote the link of $X^{(k)}$ at $x$ along with the metric induced by the given Lipschitz Riemannian metric on $X$. Note that $N$ is a spherical $(n-k-1)$ complex. Set

$$
\lambda_{1}^{N}:=\inf _{Q \in Y} \lambda_{1}\left(N, T_{Q} Y\right)
$$

where $\lambda_{1}\left(N, T_{Q} Y\right)$ denotes the first eigenvalue of the Laplacian of $N$ with values in the tangent cone of $Y$ at $Q$ (see Section 8 for further details). More precisely, our main theorem is as follows:

Main Theorem (cf. Theorem 9.1). Let B(r) be a ball of radius $r$ around a point $x$ in an admissible complex $X$ endowed with a Lipschitz Riemannian metric $g,(Y, d)$ an NPC space and $f:(B(r), g) \rightarrow(Y, d)$ a harmonic map.

(1) If $x \in X-X^{(n-2)}$, let $d$ denote the distance of $x$ to $X^{(n-2)}$. Then for $\varrho \in(0,1)$ and $d^{\prime} \leq \min \{\varrho r, \varrho d\}, f$ is Lipschitz continuous in $B\left(d^{\prime}\right)$ with Lipschitz constant depending on the total energy $E^{f}$ of the map $f,(B(r), g), d$ and $\varrho$.

(2) If $x \in X^{(k)}-X^{(k-1)}$ for $k=0, \ldots, n-2$, let d denote the distance of $x$ to $X^{(k-1)}$. Then for $\varrho \in(0,1)$ and $d^{\prime} \leq \min \{\varrho r, \varrho d\}, f$ is Hölder continuous in $B\left(d^{\prime}\right)$ with Hölder exponent and constant depending on the total energy $E^{f}$ of the map $f,(B(r), g), d$ and $\varrho$. More precisely, the Hölder exponent $\alpha$ has a lower bound given by the following: If $\lambda_{1}^{N} \geq \beta(>\beta)$ then $\alpha(\alpha+n-k-2) \geq \beta(>\beta)$. In particular, if $\lambda_{1}^{N} \geq$ $n-k-1$, then $f$ is Lipschitz continuous in a neighborhood of $x$.

The paper is organized as follows: In Section 2 we define our domain and target spaces and recall the notion of harmonic maps. In Section 3 we prove the monotonicity formula in our setting and in Section 4 we discuss the Hölder continuity of harmonic maps. Section 3 is in some sense the heart of the paper as all subsequent results depend on it. Though similar in spirit with the monotonicity formula of [9] it also differs significantly in the fact that we show that the relevant quantity (the order function) is not 
monotone as in [9]. Nonetheless, we show that the order function has a welldefined limit. This is necessary due to the fact that the different strata in the complex are not assumed to be totally geodesic. As mentioned before, in Section 4 we include a proof of the Hölder continuity of harmonic maps with a slightly more relaxed assumption on the metric of the complex than in $[7,8]$. The purpose of Sections 5 and 6 is to construct a tangent map. We then establish properties of maps from a flat domain in order to analyze the tangent map in Section 7. Finally, Section 8 is devoted to the proof of the Main Theorem.

Notation. Throughout the paper, unless mentioned otherwise $(Y, d)$ or simply $Y$ will denote an NPC metric space.

\section{Domain and target spaces}

\subsection{Local models}

We now introduce our local models which will represent a neighborhood of a point in a complex. A half space is a connected component $H$ of $\mathbf{R}^{n}-h$ where $h$ is an affine hyperplane. By a normalized half space, we will mean a half space $H$ so that the hyperplane $h$ that defines $H$ contains the origin $\overrightarrow{0}$. We say the normalized half spaces $H_{1}, \ldots, H_{\nu}$ are linearly independent if the normals to the hyperplanes $h_{1}, \ldots, h_{\nu}$ defining the half spaces are linearly independent. A wedge (or a $n$-dimensional $\nu$-wedge) $W$ is the closure of the intersection of $\nu$ number of linearly independent normalized half spaces $H_{1}, \ldots, H_{\nu}$. By its construction, every wedge is a $n$-dimensional cone in $\mathbf{R}^{n}$ with $\overrightarrow{0}$ as the vertex. Wedge angles are the angles between any pair of vectors $h_{1}, \ldots, h_{\nu}$. In particular a two-wedge has one wedge angle, and in general a $\nu$-wedge has $\frac{\nu(\nu-1)}{2}$ number of wedge angles.

A face of the wedge is an intersection $W \cap h_{i_{1}} \cap \cdots \cap h_{i_{j}}, 1 \leq i_{1} \leq \cdots \leq$ $i_{j} \leq \nu$. For example, the intersection $W \cap h_{1}$ is a face which is a $(n-1)$ dimensional linear subspace of $\mathbf{R}^{n}$ and the intersection $W \cap h_{1} \cap \cdots \cap h_{\nu}$ is a face which is a $(n-\nu)$-dimensional linear subspace of $\mathbf{R}^{n}$. This latter face is the lowest dimensional face of $W$ and we denote it by $D$. We will use the coordinates of $\mathbf{R}^{n}$ to label points in $W$. For simplicity, we always choose the coordinate system $\left(x^{1}, \ldots, x^{n}\right)$ of $\mathbf{R}^{n}$ so that $D$ is given as $x^{n-\nu+1}=\cdots=$ $x^{n}=0$.

Let $W_{1}, \ldots, W_{l}$ be $n$-dimensional $\nu$-wedges and let $\left\{F_{i}^{a}\right\}_{a=1, \ldots, \nu}$ be the set of all $(n-1)$-dimensional faces of $W_{i}$ for $i=1, \ldots, l$. For any $i, j=1, \ldots, l$ with $i \neq j$ let $\varphi_{i j}^{a b}: F_{i}^{a} \rightarrow F_{j}^{b}$ be a possibly empty linear isometry called a 
gluing map of $F_{i}^{a}$ and $F_{j}^{b}$ and let $\Phi_{i j}$ be a set of all gluing maps $\varphi_{i j}^{a b}: F_{i}^{a} \rightarrow F_{j}^{b}$ for $a, b=1, \ldots, \nu$. Let $\Phi$ be the union of $\Phi_{i j}$ for $i, j=1, \ldots, l$ and $i \neq j$.

Definition 2.1. A dimension- $n$, codimension- $\nu$ local model $\mathbf{B}=\cup W_{i} / \sim$ is a disjoint union of $n$-dimensional $\nu$-wedges $W_{1}, \ldots, W_{l}$ along with an equivalence relation $\sim$ defined by setting $x \sim x^{\prime}$ if $\varphi(x)=x^{\prime}$ for $\phi \in \Phi$. We further require (i) the cardinality of each $\Phi_{i j}$ is at most 1 and (ii) for every $i=1, \ldots, l$ and $a=1, \ldots, \nu$, there exists a non-empty gluing map in $\Phi$ with $F_{i}^{a}$ as a domain or target.

When we have two $n$-dimensional 1-wedges, i.e., two half spaces, glued together along $D=\left\{x^{n}=0\right\}$, the local model is simply $\mathbf{R}^{n}$ and this will be referred to as a codimension-0 local model. Given a face $F$ of a wedge $W_{i}$, we will also call its equivalence class in $\mathbf{B}$ a face. The boundary of a local model is the union of all $(n-1)$-dimensional faces which belong to exactly one wedge. Throughout the paper we also assume that our local models have empty boundary. Also note that property (iii) implies that our local models $\mathbf{B}$ are connected and admissible i.e., that $\mathbf{B}-F$ is connected for any $(n-2)$-dimensional face $F$.

Since $W$ is a subset of $\mathbf{R}^{n}$, there is a natural Euclidean metric inherited from $\mathbf{R}^{n}$. This defines an Euclidean metric $\delta$ on $\mathbf{B}$. For $x, y \in \mathbf{B}$, let $|x-y|$ be the induced distance function from $\delta$. Set $\mathbf{B}(r)$ be the $r$-ball centered at the origin of $\mathbf{B}$ and $W(r)=\mathbf{B}(r) \cap W$ for any wedge $W$ of $\mathbf{B}$. For the sake of simplicity, we will also refer to $W(r)$ as a wedge (of $\mathbf{B}(r)$ ). Also for $x \in \mathbf{B}$ we will denote by $B_{x}(r)$ the Euclidean $r$-ball around $x$. Note that throughout the paper all balls will be taken with respect to the metric $\delta$.

We now give examples of wedges in dimension 2 and dimension 3. (i) The only two dimensional one-wedge (up to linear isometry) is the half plane $\left\{(x, y) \in \mathbf{R}^{2}: y \geq 0\right\}$. We consider a model space $\mathbf{B}$ where $k$ copies of one-wedges are glued together along $D=\left\{(x, y) \in \mathbf{R}^{2}: y=0\right\}$. This example models a neighborhood of an edge point of a two-dimensional simplicial complex. (ii) An example of a two-dimensional two-wedge is the first quadrant $\left\{(x, y) \in \mathbf{R}^{2}: x, y \geq 0\right\}$. Another example is the set $W=\left\{(x, y) \in \mathbf{R}^{2}\right.$ : $\sqrt{3} x \geq y \geq 0\}$. A vertex point of a two-dimensional simplicial complex can be modelled by a model space where one copies of $W$ are glued together along their faces (in this case lines $y=0$ or $y=\sqrt{3} x$ ) according to the combinatorial information of the complex. Note that $D$ is this case is the point $x=y=$ 0 . (iii) The only three-dimensional one-wedge (up to linear isometry) is the half space $\left\{(x, y, z) \in \mathbf{R}^{3}: z \geq 0\right\}$. The model space $\mathbf{B}$ where $l$ copies of onewedges are glued together along $D=\left\{(x, y, z) \in \mathbf{R}^{3}: z=0\right\}$ models a neighborhood of the two-skeleton in a three-dimensional simplicial complex. (iv) 
An example of a three dimensional two-wedge is $\left\{(x, y, z) \in \mathbf{R}^{3}: y, z \geq 0\right\}$. Another example is the set $W=\left\{(x, y, z) \in \mathbf{R}^{3}: \sqrt{3} y \geq z \geq 0\right\}$. A neighborhood of a point on a one-skeleton of a three-dimensional simplicial complex can be modelled by a model space $\mathbf{B}$ where $l$ copies of $W$ are glued together along their faces according to the combinatorial information. Here, $D=$ $\left\{(x, y, z) \in \mathbf{R}^{3}: y=z=0\right\}$. (v) An example of a three-dimensional threewedge is the first octant $\left\{(x, y, z) \in \mathbf{R}^{3}: x, y, z \geq 0\right\}$. Another example is the set $W$ consisting of points of the form $\sum_{i=1}^{3} t_{i} v_{i}, t_{i} \geq 0$ where $v_{1}=(1,0,0)$, $v_{2}=\left(\frac{1}{2}, \frac{\sqrt{3}}{2}, 0\right)$ and $v_{3}=\left(\frac{1}{2}, \frac{1}{2 \sqrt{3}}, \frac{\sqrt{2}}{\sqrt{3}}\right)$. Note that the standard tetrahedron consists of points of the form $\sum_{i=1}^{3} t_{i} v_{i}, 0 \leq t_{i} \leq 1$. A neighborhood of a point on the 0 -skeleton of a three-dimensional simplicial complex can be modelled by a model space $\mathbf{B}$ where $l$ copies of $W$ are glued together along their faces according to the combinatorial information of the complex. Here, $D$ is the point $(0,0,0)$.

Let $\mathbf{B}$ be a dimension- $n$, codimension- $\nu$ local model and let $\nu=n-k$. Recall that this means that $D \subset \mathbf{B}$ is of dimension $k$; more specifically, $D$ can be isometrically identified with $\mathbf{R}^{k}$. We say $x \in \mathbf{B}$ is a codimension$(n-j)$ singular point if $B_{x}(\sigma)$ is homeomorphic to $\mathbf{B}^{\prime}(\sigma)$ where $\mathbf{B}^{\prime}$ is some dimension- $n$, codimension- $(n-j)$ local model for some $\sigma>0$. We denote the closure of the set of codimension- $(n-j)$ singular points by $S_{j}$ and set $S_{-1}=\emptyset$. For example, if $\mathbf{B}$ is a codimension- $(n-k)$ local model, then $S_{k}=$ $D$ and $S_{i}=\emptyset$ for $i=-1, \ldots, k-1$.

The following two definitions will be important in Section 4.

Definition 2.2. Suppose $x \in S_{j+1}-S_{j}$. Thus, $x$ is an interior point of a $(j+1)$-dimensional face $F$. We define $\pi_{j}(x)$ to be the set of all points $x^{\prime}$ in $S_{j} \cap F$ such that $\left|x-x^{\prime}\right|=\min _{y \in S_{j} \cap F}|x-y|$. First, note that the closest point projection of $x$ to the boundary of $F$ is not necessarily unique so that $\pi_{j}(x)$ may contain more than one point. Secondly, because a face of a local model is a convex subset of Euclidean space, $\pi_{j}(x) \subset S_{j}-S_{j-1}$. For $i>j$, $x \in S_{i}$ and $x^{\prime} \in S_{j}$, we write $x \triangleright x^{\prime}$ if we can arrive from $x$ to $x^{\prime}$ by a sequence of successive projections, i.e., there exists a sequence

$$
x=y_{i}, y_{i-1}, \ldots, y_{j+1}, y_{j}=x^{\prime}
$$

so that

$$
\begin{aligned}
y_{i} & \in S_{i}, \\
y_{i-1} & \in \pi_{i-1}\left(y_{i}\right) \subset S_{i-1}, \\
& \ldots
\end{aligned}
$$




$$
\begin{aligned}
y_{j+1} & \in \pi_{j+1}\left(y_{i-j-1}\right) \subset S_{j+1}, \\
y_{j} & \in \pi_{j}\left(y_{i-j}\right) \subset S_{j} .
\end{aligned}
$$

For $x \in \mathbf{B}(\sigma)$, let $\Pi_{j}(x)$ be the set of points $x^{\prime} \in S_{j}$ so that $x \triangleright x^{\prime}$. For any set $N$, let $\Pi_{j}(N)$ be the set of points $x^{\prime} \in S_{j}$ so that $x \triangleright x^{\prime}$ for $x \in N$.

Definition 2.3. Let $x \in \mathbf{B}$ and $\sigma>0$. The ball $B_{x}(\sigma)$ is called homogeneous if for all $t \in(0,1)$

$$
B_{x}(t \sigma)=t B_{x}(\sigma) \text {. }
$$

Given $x \in \mathbf{B}$, we let $S t(x)$ denote the star of the point $x$ in $\mathbf{B}$ i.e., the union of the wedges containing $x$. Finally, if $x \in \mathbf{B}(r)$ we set $R(x)$ to be the radius of the largest homogeneous ball centered at $x$ contained in $S t(x) \cap \mathbf{B}(r)$.

In addition to the Euclidean metric $\delta$ we equip a local model $\mathbf{B}$ (or $\mathbf{B}(r)$ ) with a Lipschitz Riemannian metric $g$. By this we mean that for each wedge $W$ of $\mathbf{B}$ (resp. each face $F$ of $\mathbf{B}$ ), we have a Lipschitz Riemannian metric $g_{W}$ (resp. $\left.g_{F}\right)$ up to the boundary of $W$ (resp. $F$ ) with the property that if $F^{\prime}$ is a face of $W$ (resp. $F$ ) then the restriction $g_{W}$ (resp. $g_{F}$ ) to $F^{\prime}$ is equal to $g_{F^{\prime}}$. Note that we do not necessarily assume that the faces of the wedges are totally geodesic. We can express $g$ as a matrix $\left(g_{i j}\right)$ in terms of the Euclidean coordinate system on the wedges $W$ inherited from the Euclidean space.

Definition 2.4. We say $\lambda \in(0,1]$ is an ellipticity constant of $g$ if for each wedge $W$ (resp. each face $F$ ) the ellipticity constants of $g_{W}$ (resp. $g_{F}$ ) are bounded below by $\lambda$ and above by $\frac{1}{\lambda}$, in other words in terms of Euclidean coordinates on $W$ (resp. $F$ ) we have

$$
\lambda^{2}|\xi|^{2} \leq \sum_{i, j=1}^{n} g_{i j} \xi^{i} \xi^{j} \leq \frac{1}{\lambda^{2}}|\xi|^{2} .
$$

Definition 2.5. We say a metric $g$ on $\mathbf{B}(r)$ is normalized if $g_{i j}(0)=\delta_{i j}$.

\subsection{Admissible cell complexes}

A convex cell complex or simply a complex $X$ in an affine space $\mathbf{E}^{\mathbf{d}}$ is a finite collection $\left\{F^{k}\right\}$ of cells where each $F^{0}$ is a point, and each $F^{k}$ is a bounded convex piecewise linear polyhedron with interior in some $\mathbf{E}^{\mathbf{k}} \subset \mathbf{E}^{\mathbf{d}}$, such that the boundary $\partial F^{k}$ of $F^{k}$ is a union of $F^{s}$ with $s<k$ (called the faces of $F^{k}$ ), and such that if $s<k$ and $F^{k} \cap F^{s} \neq \varnothing$, then $F^{s} \subset F^{k}$. For 
example a simplicial complex is a cell complex whose cells are all simplices. We will denote by $X^{(i)}$ the $i$-dimensional skeleton of $X$, i.e., the union of all cells $F^{k}$ where $k \leq i$. $X$ is called $n$-dimensional or simply a $n$-complex if $X^{(n+1)}=\emptyset$ but $X^{(n)} \neq \emptyset$. The boundary of $X$, denoted $\partial X$, is the union of $k$-cells $F^{k}, k<n$, so that $F$ is a face of exactly one $n$-cell. A point $p \in \partial X$ is called a boundary point and a point $p \in X-\partial X$ is called an interior point. In the sequel, we will require the following conditions (sometimes called admissibility conditions, or admissible complex (cf.[7])):

(1) $X$ is dimensionally homogeneous; i.e., for $k<n$, each $k$-cell is a face of a $n$-cell.

(2) $X$ is locally $(n-1)$-chainable; i.e., for every connected, open set $U \subset$ $X$, the open set $U-X^{(n-2)}$ is connected.

A Lipschitz Riemannian $n$-complex is a convex cell complex where each cell $F$ is equipped with a Lipschitz Riemannian metric $g_{F}$ up to the boundary. We are assuming that if $F^{\prime}$ is a face of $F$ then the restriction $g_{F}$ to $F^{\prime}$ is equal to $g_{F^{\prime}}$. Admissible cell complexes are based on local models because of the following obvious proposition

Proposition 2.1. Let $X$ be an admissible Lipschitz Riemannian complex of dimension $n$ with metric $g$ given as $\left(g_{i j}\right)$. Let $x \in X^{(k)}-X^{(k-1)}$ and let $\lambda \in$ $(0,1]$ be the ellipticity constant of $g$ near $x$. Then there exist a dimension- $n$, codimension- $(n-k)$ local model $\mathbf{B}$ and a homeomorphism $L_{x}: \mathbf{B}(\lambda R(x)) \rightarrow$ $L_{x}(\mathbf{B}(\lambda R(x))) \subset X$ so that

(i) $L_{x}(0)=x$,

(ii) for any wedge $W$ of $\mathbf{B}, L_{x}$ restricted to $W \cap \mathbf{B}(\lambda R(x))$ maps into the closure $\bar{F}$ of a n-cell of $X$,

(iii) with $W$ viewed as a subset of $\mathbf{R}^{n}$ as in Section 2.1, $\left.L_{x}\right|_{W \cap \mathbf{B}(\lambda R(x))}$ uniquely extends as an affine map $\mathcal{L}_{x}$ defined on $\mathbf{R}^{n}$,

(iv) the pullback metric $h=L_{x}^{*} g$ has the property that $h_{i j}(0)=\delta_{i j}$ with respect to the coordinate chart on $W$.

Because $g$ has ellipticity constant $\lambda, \mathcal{L}_{x}$ maps the ball of radius $\lambda R(x)$ centered at 0 into the largest ellipse contained in the ball of radius $R(x)$ centered at $x$.

We now mention that (a) trees and Bruhat-Tits buildings are examples of admissible cell complexes; (b) for any finitely generated group $\Gamma$ there is a two-dimensional admissible complex without boundary whose fundamental 
group is $\Gamma$ (cf.[4]); (c) triangulable Lipschitz manifolds and normal complex analytic spaces are homeomorphic to admissible complexes. For more details we refer to [7].

\subsection{Harmonic maps}

We now define our target spaces.

Definition 2.6. A complete metric space $(Y, d)$ is said to be an NPC (nonpositively curved) space if the following conditions are satisfied:

(i) The space $(Y, d)$ is a length space. That is, for any two points $P$ and $Q$ in $Y$, there exists a rectifiable curve $\gamma_{P Q}$ so that the length of $\gamma_{P Q}$ is equal to $d(P, Q)$ (which we will sometimes denote by $d_{P Q}$ for simplicity). We call such distance realizing curves geodesics.

(ii) Let $P, Q, R \in Y$. Define $Q_{t}$ to be the point on the geodesic $\gamma_{Q R}$ satisfying $d_{Q Q_{t}}=t d_{Q R}$ and $d_{Q_{t} R}=(1-t) d_{Q R}$. Then

$$
d_{P Q_{t}}^{2} \leq(1-t) d_{P Q}^{2}+t d_{P R}^{2}-t(1-t) d_{Q R}^{2} .
$$

Remark. Simply connected Riemannian manifolds of non-positive sectional curvature, Bruhat-Tits Euclidean buildings associated with actions of $p$-adic Lie groups and $\mathbf{R}$-trees are examples of NPC spaces. These spaces are also referred to as $\operatorname{CAT}(0)$ spaces in literature. We refer to [1] for more details.

We will now review the definition of harmonic maps. For details we refer the reader to [7]. First, we define the energy of a map. Let $(Y, d)$ be an NPC space and $f:(\mathbf{B}(r), g) \rightarrow Y$ be a $L^{2}$ map from the local model to $Y$. The energy ${ }^{g} E^{f}$ is defined as the weak limit of the $\epsilon$-approximate energy density measures which are measures derived from the appropriate average difference quotients. More specifically, define the $\epsilon$-approximate energy $e_{\epsilon}: \mathbf{B}(r) \rightarrow \mathbf{R}$ by

$$
e_{\epsilon}(x)= \begin{cases}\int_{y \in S(x, \epsilon)} \frac{d^{2}(f(x), f(y))}{\epsilon^{2}} \frac{d \sigma_{x, \epsilon}}{\epsilon^{n-1}} & \text { for } x \in \mathbf{B}(r)_{\epsilon}, \\ 0 & \text { for } x \in \mathbf{B}(r)-\mathbf{B}(r)_{\epsilon}\end{cases}
$$

where $\sigma_{x, \epsilon}$ is the induced measure on the $\epsilon$-sphere $S(x, \epsilon)$ centered at $x$ and $\mathbf{B}(r)_{\epsilon}=\{x \in \mathbf{B}(r): d(x, \partial \mathbf{B}(r))>\epsilon\}$. Define a family of functionals ${ }^{g} E_{\epsilon}^{f}$ : 
$C_{c}(\mathbf{B}(r)) \rightarrow \mathbf{R}$ by setting

$$
{ }^{g} E_{\epsilon}^{f}(\varphi)=\int_{\mathbf{B}(r)} \varphi e_{\epsilon} d \mu_{g}
$$

Definition 2.7. We say that $f:(\mathbf{B}(r), g) \rightarrow Y$ has finite energy (or that $f \in W^{1,2}(\mathbf{B}(r), Y)$ or simply that $f$ is a $W^{1,2}$ map) if

$$
{ }^{g} E^{f}:=\sup _{\varphi \in C_{c}(\mathbf{B}(r)), 0 \leq \varphi \leq 1} \limsup _{\epsilon \rightarrow 0}{ }^{g} E_{\epsilon}^{f}(\varphi)<\infty
$$

Theorem 2.1. Suppose $f:(\mathbf{B}(r), g) \rightarrow Y$ has finite energy. Then the measures $e_{\epsilon}(x) d x$ converge weakly to a measure which is absolutely continuous with respect to the Lebesgue measure. Therefore, there exists a function $e(x)$, which we call the energy density, so that $e_{\epsilon}(x) d \mu_{g} \rightarrow e(x) d \mu_{g}$.

In analogy to the case of real valued functions, we write $|\nabla f|_{g}^{2}(x)$ in place of $e(x)$. (We will omit the subscript in $|\nabla f|_{g}^{2}, d \mu_{g}$, etc. if it is clear which metric we are using). In particular,

$$
{ }^{g} E^{f}=\int_{\mathbf{B}(r)}|\nabla f|_{g}^{2} d \mu_{g} .
$$

For a set $S \subset \mathbf{B}$, let

$$
{ }^{g} E^{f}[S]=\int_{S}|\nabla f|_{g}^{2} d \mu_{g}
$$

We also define

$$
|\nabla f|_{g}(x)=\left(|\nabla f|_{g}^{2}(x)\right)^{1 / 2}
$$

For a Lipschitz vector field $V$ on $\mathbf{B}(r),\left|f_{*}(V)\right|_{g}^{2}$ is similarly defined. The real valued $L^{1}$ function $\left|f_{*}(V)\right|_{g}^{2}$ generalizes the norm squared on the directional derivative of $f$. We refer to $[7,11]$ for more details.

Theorem 2.2. Suppose $f:(\mathbf{B}(r), g) \rightarrow Y$ has finite energy and $V$ is a Lipschitz vector field. The operator ${ }^{g} \pi^{f}$ defined by

$$
{ }^{g} \pi^{f}(V, W)=\frac{1}{2}\left|f_{*}(V+W)\right|_{g}^{2}-\frac{1}{2}\left|f_{*}(V-W)\right|_{g}^{2}
$$

is continuous, symmetric, bilinear, non-negative and tensorial. We call ${ }^{g} \pi^{f}$ the pull-back metric. 
Notation 2.1. Let $\left\{\frac{\partial}{\partial x^{1}}, \ldots, \frac{\partial}{\partial x^{n}}\right\}$ be the standard Euclidean basis defined on each wedge inherited from $\mathbf{R}^{n}$ and $\delta$ the standard Euclidean metric. Set

$$
\frac{\partial f}{\partial x^{i}} \cdot \frac{\partial f}{\partial x^{j}}={ }^{\delta} \pi^{f}\left(\frac{\partial}{\partial x^{i}}, \frac{\partial}{\partial x^{j}}\right) \text { and }\left|\frac{\partial f}{\partial x^{i}}\right|^{2}=\frac{\partial f}{\partial x^{i}} \cdot \frac{\partial f}{\partial x^{i}} .
$$

Similarly for the standard Euclidean polar coordinates $\left(r, \theta_{1}, \ldots, \theta_{n-1}\right)$ on each wedge we denote

$$
\frac{\partial f}{\partial x^{k}} \cdot \frac{\partial f}{\partial r}={ }^{\delta} \pi^{f}\left(\frac{\partial}{\partial x^{k}}, \frac{\partial}{\partial r}\right),\left|\frac{\partial f}{\partial r}\right|^{2}=\frac{\partial f}{\partial r} \cdot \frac{\partial f}{\partial r}={ }^{\delta} \pi^{f}\left(\frac{\partial}{\partial r}, \frac{\partial}{\partial r}\right)
$$

and

$$
\frac{\partial f}{\partial \theta_{i}} \cdot \frac{\partial f}{\partial \theta_{j}}={ }^{\delta} \pi^{f}\left(\frac{\partial}{\partial \theta_{i}}, \frac{\partial}{\partial \theta_{j}}\right) .
$$

Note that the energy density with respect to the metric $g$ is given by

$$
|\nabla f|_{g}^{2}=\sum_{i, j} g^{i j} \frac{\partial f}{\partial x^{i}} \cdot \frac{\partial f}{\partial x^{j}}
$$

whereas the energy density with respect to the Euclidean metric is given by

$$
|\nabla f|^{2}=|\nabla f|_{\delta}^{2}=\sum_{i}\left|\frac{\partial f}{\partial x^{i}}\right|^{2} .
$$

By using the identification with local models given in Proposition 2.1 all the above notions extend for any admissible complex $X$ replacing $\mathbf{B}$ as a domain. We omit the details.

For the trace of $W^{1,2}$ maps we refer to the following theorem (cf. [7, 11]).

Theorem 2.3. Let $\Omega$ be a compact domain in an admissible complex with Lipschitz Riemannian metric $g$ and $(Y, d)$ a metric space. Any $f \in W^{1,2}$ $(\Omega, Y)$ has a well-defined trace map denoted by $\operatorname{Tr}(f)$ or simply $f$, with $\operatorname{Tr}(f) \in L^{2}(\partial \Omega, Y)$. If the sequence $f_{i} \in W^{1,2}(\Omega, Y)$ has uniformly bounded energies ${ }^{g} E^{f_{i}}[\Omega]$ and if $f_{i}$ converges in $L^{2}$ to a map $f$, then $\operatorname{Tr}\left(f_{i}\right)$ converges to $\operatorname{Tr}(f)$ in $L^{2}(\partial \Omega, Y)$. Two maps $f, g \in W^{1,2}(\Omega, Y)$ have the same trace if and only if $d(f, g) \in W^{1,2}(\Omega, \mathbf{R})=W^{1,2}(\Omega)$ has trace zero.

We define $W_{0}^{1,2}(\Omega)$ to be the subset of $W^{1,2}(\Omega)$ functions with trace zero. The next two theorems are also contained in $[7,11]$. 
Theorem 2.4. Let $\Omega$ be a compact domain in an admissible complex with Lipschitz Riemannian metric $g$ and $(Y, d)$ a locally compact metric space. Let $f_{i} \in W^{1,2}(\Omega, Y)$ be a sequence satisfying $f_{i} \rightarrow f$ in $L^{2}$ and ${ }^{g} E^{f_{i}}[\Omega] \leq C$ for some constant $C$ independent of $i$. Then

$$
{ }^{g} E^{f}[\Omega] \leq \liminf _{i \rightarrow \infty}{ }^{g} E^{f_{i}}[\Omega]
$$

Theorem 2.5. Let $\Omega$ be a compact domain in anadmissible complex with Lipschitz Riemannian metric $g$ and $(Y, d)$ a locally compact metric space. Let $f_{i} \in W^{1,2}(\Omega, Y)$ be a sequence satisfying ${ }^{g} E^{f_{i}}[\Omega]+\int_{X} d^{2}\left(f_{i}(x), Q\right) d \mu_{g}(x) \leq$ $C$ for some fixed point $Q$ of $Y$ and some constant $C$ independent of $i$. Then, there is a subsequence of $f_{i}$ that converges in $L^{2}(X, Y)$ to a finite energy map $f$.

Definition 2.8. Let $\Omega$ be a compact domain in an admissible complex with Lipschitz Riemannian metric $g$ and $(Y, d)$ an NPC space. A map $f: \Omega \rightarrow Y$ is said to be harmonic if it is energy minimizing among all $W^{1,2}$-maps with the same trace (boundary condition).

We end this section by proving two versions of the Poincaré inequality that we will need in the sequel.

Theorem 2.6. Let $\Omega$ be a compact domain in an admissible complex with Lipschitz Riemannian metric $g$. Then, there is a constant $C$ depending only on $\Omega$ and $g$ so that for any $\varphi \in W_{0}^{1,2}(\Omega)$

$$
\int_{\Omega} \varphi^{2} d \mu_{g} \leq C \int_{\Omega}|\nabla \varphi|_{g}^{2} d \mu_{g} .
$$

Proof. The proof follows closely the proof of the Poincare inequality in $[16$, Lemma 2], therefore we will only give a sketch. Suppose the assertion is false: then for each $i=1,2, \ldots$, there exist functions $\varphi_{i} \in W_{0}^{1,2}(\Omega)$ so that

$$
\int_{\Omega}\left|\nabla \varphi_{i}\right|_{g}^{2} d \mu_{g}<\frac{1}{i} \int_{\Omega} \varphi_{i}^{2} d \mu_{g}
$$

By setting

$$
v_{i}=\frac{\varphi_{i}}{\left(\int_{\Omega} \varphi_{i}^{2} d \mu_{g}\right)^{1 / 2}}
$$

we have $\int_{\Omega} v_{i}^{2} d \mu_{g}=1$ and $\int_{\Omega}\left|\nabla v_{i}\right|_{g}^{2} d \mu_{g}<\frac{1}{i}$. By Theorems 2.5 and 2.4 there exists a subsequence (which we denote again by $i$ ) so that $v_{i} \rightarrow v$ in $L^{2}(\Omega)$ 
and $\int_{\Omega}|\nabla v|_{g}^{2} d \mu_{g} \leq \liminf _{i \rightarrow \infty} \int_{\Omega}\left|\nabla v_{i}\right|_{g}^{2} d \mu_{g}=0$. This implies that $v$ must be constant, and since $\int_{\Omega} v^{2} d \mu_{g}=\lim _{i \rightarrow \infty} \int_{\Omega} v_{i}^{2} d \mu_{g}=1$ it must be nonzero. On the other hand, Theorem 2.3 implies that the trace of $v$ is 0 , which is a contradiction.

Theorem 2.7. Let $X$ be a compact admissible complex with a Lipschitz Riemannian metric $g$ and $(Y, d)$ a metric space. Then, there is a constant $C$ depending only on $X$ and the ellipticity constant of the metric $g$ so that for any $\varphi \in W^{1,2}(X, Y)$

$$
\inf _{P \in Y} \int_{X} d^{2}(\varphi, P) d \mu_{g} \leq C \int_{X}|\nabla \varphi|_{g}^{2} d \mu_{g}
$$

Proof. By Fugledge [8], proof of Corollary 1 Step 2, the Poincare inequality holds for the Euclidean metric, i.e., there exists a constant $C$ depending only on $X$ so that

$$
\inf _{P \in Y} \int_{X} d^{2}(\varphi, P) d \mu \leq C \int_{X}|\nabla \varphi|^{2} d \mu .
$$

Let $c$ be a universal constant depending only on the dimension of $X$. It follows from (2.1) that

$$
\sum_{i, j=1}^{n} \delta_{i j} \frac{\partial \varphi}{\partial x_{i}} \frac{\partial \varphi}{\partial x_{j}} \leq c \lambda^{-2} \sum_{i, j=1}^{n} g^{i j} \frac{\partial \varphi}{\partial x_{i}} \frac{\partial \varphi}{\partial x_{j}}
$$

and hence

$$
|\nabla \varphi|^{2} \leq c \lambda^{-2}|\nabla \varphi|_{g}^{2}
$$

Furthermore, (2.1) also implies that

$$
\lambda^{n} \leq \frac{d \mu_{g}}{d \mu} \leq \lambda^{-n}
$$

which combined with (2.2) completes the proof.

Corollary 2.1. Let B be a dimension-n, codimension- $\nu$ local model, $g$ a Lipschitz Riemannian metric defined on $\mathbf{B}(r)$ and $(Y, d)$ a metric space. 
Then, there is a constant $C$ depending only on $\mathbf{B}(r)$ and the ellipticity constant of $g$ so that for any $\varphi \in W^{1,2}(\mathbf{B}(r), Y)$ and $\sigma$ sufficiently small

$$
\inf _{P \in Y} \int_{\mathbf{B}(\sigma)} d^{2}(\varphi, P) d \mu_{g} \leq C \sigma^{2} \int_{\mathbf{B}(\sigma)}|\nabla \varphi|_{g}^{2} d \mu_{g} .
$$

Similarly, for any $\varphi \in W^{1,2}(\partial \mathbf{B}(r), Y)$ and $\sigma$ sufficiently small

$$
\inf _{P \in Y} \int_{\partial \mathbf{B}(\sigma)} d^{2}(\varphi, P) d \Sigma_{g} \leq C \sigma^{2} \int_{\partial \mathbf{B}(\sigma)}\left|\nabla^{\partial} \varphi\right|_{g}^{2} d \Sigma_{g},
$$

where $\nabla^{\partial}$ is the gradient tangential to $\partial \mathbf{B}(\sigma)$.

Proof. Both inequalities follow immediately from Theorem 2.7 by rescaling and by the fact that $\mathbf{B}(\sigma)$ (resp. $\partial \mathbf{B}(\sigma)$ ) is piecewise smoothly diffeomorphic to the star (resp. the link) of the point 0 .

\section{Monotonicity formula}

In this section, we prove a monotonicity formula for harmonic maps. This is a modified version of the monotonicity formula shown in [9] where the domain space is a Riemannian manifold. The technical difficulties posed by the singular nature of the domain space considered in this paper is that we cannot necessarily work in normal coordinates and that the faces are not necessarily totally geodesic in wedges with respect to the metric given.

Let $\mathbf{B}$ be a local model. We continue to use the Euclidean coordinates $\left(x^{1}, \ldots, x^{n}\right)$ in each wedge $W$. For $x, y \in \mathbf{B}$, we denote the induced (Euclidean) distance by $|x-y|$. By definition, if $x=\left(x^{1}, \ldots, x^{n}\right)$ and $y=$ $\left(y^{1}, \ldots, y^{n}\right)$ are on the same wedge of $\mathbf{B}$, then $|x-y|=$ $\sqrt{\left(x^{1}-y^{1}\right)^{2}+\cdots+\left(x^{n}-y^{n}\right)^{2}}$. Furthermore, we let $\left(r, \theta_{1}, \ldots, \theta_{n-1}\right)$ be the corresponding polar coordinates, i.e., $r$ gives the radial distance from the origin and $\theta=\left(\theta_{1}, \ldots, \theta_{n-1}\right)$ are the coordinates on the standard $(n-1)$ sphere. Let $g$ be a normalized Lipschitz metric defined on $\mathbf{B}(r)=\{x \in \mathbf{B}$ : $|x|<r\}$, i.e., if $g=\left(g_{i j}\right)$ with respect to the coordinates $x=\left(x^{1}, \ldots, x^{n}\right)$ on a wedge $W$, then

$$
\left|g_{i j}(x)-g_{i j}(\bar{x})\right| \leq c|x-\bar{x}|, \quad \forall x, \bar{x} \in W
$$

and

$$
\left|g_{i j}(x)-\delta_{i j}\right| \leq c \sigma
$$


for $|x| \leq \sigma$. For $\sigma \in(0, r)$, we set

$$
{ }^{g} E^{f}(\sigma)=\left.\int_{\mathbf{B}(\sigma)}|\nabla f|\right|_{g} ^{2} d \mu_{g}
$$

and

$$
{ }^{g} I^{f}(\sigma, Q)=\int_{\partial \mathbf{B}(\sigma)} d^{2}(f, Q) d \Sigma_{g}
$$

for $Q \in Y$. Here $d \Sigma_{g}$ is the measure on $\partial \mathbf{B}(\sigma)$ induced by $g$. By Korevaar and Schoen [11, Lemma 2.5.1], there exists a unique point $Q_{\sigma} \in Y$ so that

$$
{ }^{g} I^{f}\left(\sigma, Q_{\sigma}\right)=\inf _{Q \in Y}{ }^{g} I^{f}(\sigma, Q)
$$

Notation 3.1. For the rest of this section we will use the notation

$$
E(\sigma)={ }^{g} E^{f}(\sigma) \text { and } I(\sigma)=I(\sigma, Q)={ }^{g} I^{f}(\sigma, Q),
$$

if $Q$ is a generic point. Furthermore in all statements up to (including) Corollary 3.2, we will make the additional assumption that the metric $g$ is normalized.

If we assume that the domain is a Riemannian manifold and replace $\mathbf{B}(\sigma)$ by a geodesic $\sigma$-ball, it is shown in [9] that

$$
\sigma \mapsto e^{C \sigma} \frac{\sigma E(\sigma)}{I(\sigma)}
$$

is a non-decreasing function where $C$ is some constant depending on the metric. Note that in our case $\mathbf{B}(\sigma)$ is a $\sigma$-ball with respect to the Euclidean metric $\delta$ on $\mathbf{B}$. The reason Euclidean balls are considered here is the possible incompatibility of the induced distance functions of the metrics given on two different wedges along a shared face. More specifically, let $g_{1}$ and $g_{2}$ be the metrics defined on wedges $W_{1}$ and $W_{2}$ sharing a face $F$. Since we do not assume that $F$ is totally geodesic in $W_{1}$ or $W_{2}$, the induced distance functions in $W_{1}$ and $W_{2}$ do not necessarily agree in $F$.

We are thus considering a general Lipschitz metric $g$ with no restriction on the faces and this leads to a modified version of the monotonicity formula which in turn gives a well-defined version of the order (cf. Corollary 3.2). For a model space $\mathbf{B}$ with a Euclidean metric $\delta$, the monotonicity of (3.5) follows from [13]. 
We say a continuous function $\eta$ defined on $\mathbf{B}(r)$ is smooth if the restriction of $\eta$ to each wedge $W$ of $\mathbf{B}(r)$ is smooth up to the boundary of $W$. The set of smooth functions with compact support in $\mathbf{B}(r)$ will be denoted by $C_{c}^{\infty}(\mathbf{B}(r))$.

Lemma 3.1. Let $f:(\mathbf{B}(r), g) \rightarrow(Y, d)$ be a harmonic map. For any $\sigma \in$ $(0, r)$ and $\eta \in C_{c}^{\infty}(\mathbf{B}(\sigma))$,

$$
\begin{gathered}
\int_{\mathbf{B}(\sigma)}\left(|\nabla f|_{g}^{2}(2-n) \eta-|\nabla f|_{g}^{2} \sum_{i} x^{i} \frac{\partial \eta}{\partial x^{i}}\right. \\
\left.+2 \sum_{i, j, k} g^{i k} \frac{\partial \eta}{\partial x^{i}} x^{j} \frac{\partial f}{\partial x^{j}} \cdot \frac{\partial f}{\partial x^{k}}\right) d \mu_{g} \\
+O(\sigma) E(\sigma)=0
\end{gathered}
$$

where $|O(\sigma)| \leq c \sigma$ and $c$ depends on $\mathbf{B}(r)$ and the Lipschitz bound of $g$.

Proof. For $t$ sufficiently small, we define $F_{t}: \mathbf{B}(r) \rightarrow \mathbf{B}(r)$ by setting

$$
F_{t}(x)=(1+t \eta(x)) x
$$

for each $x=\left(x^{1}, \ldots, x^{n}\right)$ in a wedge $W$. For $f_{t}: \mathbf{B}(r) \rightarrow Y$ defined as $f_{t}=$ $f \circ F_{t}$, a direct computation (cf.[9, Section 2]) on each wedge $W$ of $\mathbf{B}(r)$ gives

$$
\begin{aligned}
& \left.\frac{d}{d t}{ }^{g} E^{f_{t}}[W]\right|_{t=0} \\
& =\int_{W}\left(|\nabla f|_{g}^{2}(2-n) \eta-|\nabla f|_{g}^{2} \sum_{i} x^{i} \frac{\partial \eta}{\partial x^{i}}\right. \\
& \left.\quad+2 \sum_{i, j, k} g^{i k} \frac{\partial \eta}{\partial x^{i}} x^{j} \frac{\partial f}{\partial x^{j}} \cdot \frac{\partial f}{\partial x^{k}}\right) d \mu_{g} \\
& + \text { remainder. }
\end{aligned}
$$

Here, the remainder term is given by

$$
\int_{W}\left(-\eta \sum_{i, j, k} \frac{\partial g^{i j}}{\partial x^{k}} x^{k} \frac{\partial f}{\partial x^{i}} \cdot \frac{\partial f}{\partial x^{j}} \sqrt{g}+|\nabla f|_{g}^{2} \eta \sum_{i} x^{i} \frac{\partial \sqrt{g}}{\partial x^{i}}\right) d x
$$


Since we assume the metric $g$ is Lipschitz, there exists a constant $c$ so that $\left|\frac{\partial g^{i j}}{\partial x^{k}}\right|,\left|\frac{\partial \sqrt{g}}{\partial x^{i}}\right| \leq c$, which then implies that the remainder term is bounded by $c \sigma \times E(\sigma)$. Summing over all the wedges $W$ of $\mathbf{B}(r)$ we get the right-hand side of (3.6) and this equals to 0 since $f_{0}=f$ is harmonic.

Lemma 3.2. If $f:(\mathbf{B}(r), g) \rightarrow(Y, d)$ satisfies (3.6), then for $\sigma \in(0, r)$

$$
\left|\frac{E^{\prime}(\sigma)}{E(\sigma)}-\frac{n-2}{\sigma}-\frac{2}{E(\sigma)} \int_{\partial \mathbf{B}(\sigma)} \sum_{i, k} g^{i k} \frac{x^{i}}{|x|} \frac{\partial f}{\partial x^{k}} \cdot \frac{\partial f}{\partial r} d \Sigma_{g}\right| \leq c_{1}
$$

for some constant $c_{1}$ depending on $\mathbf{B}(r)$ and the Lipschitz bound of $g$,

$$
E^{\prime}(\sigma)=(1+O(\sigma))\left(\frac{n-2+O(\sigma)}{\sigma} E(\sigma)+2 \int_{\partial \mathbf{B}(\sigma)}\left|\frac{\partial f}{\partial r}\right|^{2} d \Sigma_{g}\right)
$$

and

$$
(1+c \sigma) \frac{E^{\prime}(\sigma)}{E(\sigma)} \geq \frac{n-2}{\sigma}+\frac{2}{E(\sigma)} \int_{\partial \mathbf{B}(\sigma)}\left|\frac{\partial f}{\partial r}\right|^{2} d \Sigma_{g}-c_{1}
$$

where $c$ is as in (3.1).

Proof. Let $\eta$ in (3.6) approximate the characteristic function of $\mathbf{B}(\sigma)$ to obtain

$$
E^{\prime}(\sigma)-\frac{n-2+O(\sigma)}{\sigma} E(\sigma)-2 \int_{\partial \mathbf{B}(\sigma)} \sum_{i, j, k} g^{i k} \frac{x^{i}}{|x|} \frac{\partial f}{\partial x^{k}} \cdot \frac{\partial f}{\partial r} d \Sigma_{g}=0
$$

which immediately implies inequality (3.7). Next, we use the inequality $g^{i k} \leq$ $\delta^{i k}+c \sigma$ to show

$$
\sum_{i, k} g^{i k} \frac{x^{i}}{|x|} \frac{\partial f}{\partial x^{k}} \cdot \frac{\partial f}{\partial r} \leq\left|\frac{\partial f}{\partial r}\right|^{2}+c \sigma \sum_{k}\left|\frac{\partial f}{\partial x^{k}} \cdot \frac{\partial f}{\partial r}\right| \leq\left|\frac{\partial f}{\partial r}\right|^{2}+c \sigma|\nabla f|^{2} .
$$

Using this, inequalities (3.8) and (3.9) follow again from (3.10). 
Lemma 3.3. Let $f:(\mathbf{B}(r), g) \rightarrow(Y, d)$ be a harmonic map. For any $Q \in Y$, $\triangle d^{2}(f, Q)-2|\nabla f|_{g}^{2} \geq 0$ weakly, i.e.,

$$
2 \int_{\mathbf{B}(r)}|\nabla f|_{g}^{2} \eta d \mu_{g} \leq-\int_{\mathbf{B}(r)}<\nabla d^{2}(f, Q), \nabla \eta>_{g} d \mu_{g}
$$

for any $\eta \in C_{c}^{\infty}(\mathbf{B}(r))$.

Proof. This inequality follows from a target variation of the harmonic map and hence the singular nature of the domain is not essential in the proof. Details can be found in the proof of [9, Proposition 2.2.]

Lemma 3.4. If $f:(\mathbf{B}(r), g) \rightarrow(Y, d)$ satisfies (3.11), then

$$
E(\sigma) \leq I(\sigma)^{\frac{1}{2}}\left(\left(\int_{\partial \mathbf{B}(\sigma)}\left|\frac{\partial f}{\partial r}\right|^{2} d \sigma_{g}\right)^{\frac{1}{2}}+c \sigma\left(E^{\prime}(\sigma)\right)^{\frac{1}{2}}\right)
$$

and

$$
\left.2 E(\sigma) \leq \int_{\partial \mathbf{B}(\sigma)} \frac{\partial}{\partial r} d^{2}(f, Q)\right) d \Sigma_{g}+I(\sigma)+k \sigma^{2} E^{\prime}(\sigma)
$$

for some constants $c, k$ depending on $\mathbf{B}(r)$ and the Lipschitz bound of $g$ and

$$
\frac{1}{I(\sigma)} \int_{\partial \mathbf{B}(\sigma)} \frac{\partial}{\partial r} d^{2}(f, Q) d \Sigma_{g} \leq \frac{2}{E(\sigma)} \int_{\partial \mathbf{B}(\sigma)}\left|\frac{\partial f}{\partial r}\right|^{2} d \mu_{g}+2 c \sigma \frac{E^{\prime}(\sigma)}{E(\sigma)}
$$

where $c$ is as in (3.1).

Proof. Let $\eta$ in (3.11) approximate the characteristic function of $\mathbf{B}(\sigma)$ to obtain

$$
2 E(\sigma) \leq \int_{\partial \mathbf{B}(\sigma)}<\nabla d^{2}(f, Q), \nabla|x|>_{g} d \Sigma_{g}=\int_{\partial \mathbf{B}(\sigma)} g^{i j} \frac{\partial}{\partial x^{i}} d^{2}(f, Q) \frac{x^{j}}{|x|} d \Sigma_{g} .
$$


Using the estimate $g^{i j} \leq \delta^{i j}+c \sigma$, we obtain

$$
\begin{aligned}
2 E(\sigma) \leq & \left.\int_{\partial \mathbf{B}(r)} \frac{\partial}{\partial r} d^{2}(f, Q)\right) d \Sigma_{g} \\
& \left.+c \sigma \int_{\partial \mathbf{B}(r)} \sum_{i} \mid \frac{\partial}{\partial x^{i}} d^{2}(f, Q)\right) \mid d \Sigma_{g} \\
\leq & \left.2 \int_{\partial \mathbf{B}(r)} d(f, Q) \frac{\partial}{\partial r} d(f, Q)\right) d \Sigma_{g} \\
& +2 c \sigma \int_{\partial \mathbf{B}(\sigma)} d(f, Q) \sum_{i}\left|\frac{\partial}{\partial x^{i}} d(f, Q)\right| d \Sigma_{g} \\
\leq & \left.\left.2 I(\sigma)^{\frac{1}{2}}\left(\int_{\partial \mathbf{B}(r)} \mid \frac{\partial}{\partial r} d(f, Q)\right)\right|^{2} d \Sigma_{g}\right)^{\frac{1}{2}} \\
& +2 c \sigma I(\sigma)^{\frac{1}{2}}\left(\int_{\partial \mathbf{B}(\sigma)} \sum_{i}\left|\frac{\partial}{\partial x^{i}} d(f, Q)\right|^{2} d \Sigma_{g}\right)^{\frac{1}{2}} .
\end{aligned}
$$

The triangle inequality implies that

$$
\left|\frac{\partial}{\partial x^{i}} d(f, Q)\right|^{2} \leq\left|\frac{\partial f}{\partial x^{i}}\right|^{2} \text { and } \quad\left|\frac{\partial}{\partial r} d(f, Q)\right|^{2} \leq\left|\frac{\partial f}{\partial r}\right|^{2}
$$

From this, (3.12) follows immediately. Additionally, use the CauchySchwarz inequality to obtain

$$
2 c \sigma d(f, Q)\left|\frac{\partial}{\partial x^{i}} d(f, Q)\right| \leq d^{2}(f, Q)+c^{2} \sigma^{2}\left|\frac{\partial f}{\partial x^{i}}\right|^{2}
$$

which implies

$$
\begin{aligned}
\left.2 E(\sigma) \leq \int_{\mathbf{B}(r)} \frac{\partial}{\partial r} d^{2}(f, Q)\right) d \Sigma_{g} & +\int_{\partial \mathbf{B}(\sigma)} d^{2}(f, Q) d \Sigma_{g} \\
& +c^{2} \sigma^{2} \int_{\partial \mathbf{B}(\sigma)}|\nabla f|^{2} d \Sigma_{g}
\end{aligned}
$$


From this, (3.13) follows immediately. Lastly, again use (3.15) to obtain

$$
\begin{aligned}
& \left.E(\sigma) \int_{\partial \mathbf{B}(\sigma) \frac{\partial}{\partial r} d^{2}(f, Q) d \Sigma_{g}} d(f, Q) \frac{\partial}{\partial r} d(f, Q) d \Sigma_{g}\right)^{2} \\
& \leq 2\left(\int_{\partial \mathbf{B}(\sigma)} d(f, Q) \frac{\partial}{\partial r} d(f, Q) d \Sigma_{g}\right) \\
& +2 c \sigma\left(\int_{\partial \mathbf{B}(\sigma)} d\left(\int_{\partial \mathbf{B}(\sigma)} d(f, Q) \sum_{i}\left|\frac{\partial}{\partial x^{i}} d(f, Q)\right| d \Sigma_{g}\right)\right. \\
& \quad \times 2 I(\sigma) \int_{\partial \mathbf{B}(\sigma)}\left|\frac{\partial f}{\partial r}\right|^{2} d \Sigma_{g}+2 c \sigma I(\sigma) \int_{\partial \mathbf{B}(\sigma)}|\nabla f|^{2} d \Sigma_{g} .
\end{aligned}
$$

This immediately implies (3.14).

The following energy growth estimate is also given in [2] with geodesic balls (and not Euclidean balls as it is here).

Lemma 3.5. Let $f:(\mathbf{B}(r), g) \rightarrow(Y, d)$ be a harmonic map. There exist $\sigma_{0}>0$ and $\gamma>0$ depending on $\mathbf{B}(r)$, the Lipschitz bound and the ellipticity constant of $g$ so that

$$
\sigma \mapsto \frac{E(\sigma)}{\sigma^{n-2+2 \gamma}}, \sigma \in\left(0, \sigma_{0}\right)
$$

is non-decreasing.

Proof. Let $Q_{\sigma} \in Y$ so that

$$
I\left(\sigma, Q_{\sigma}\right)=\inf _{Q \in Y} I(\sigma, Q)
$$

Thus, the Poincaré inequality (cf. Corollary 2.1) implies that there exists $C_{0}>0$ so that

$$
I\left(\sigma, Q_{\sigma}\right) \leq C_{0} \sigma^{2} \int_{\partial \mathbf{B}(\sigma)}\left|\nabla^{\partial} f\right|_{g}^{2} d \Sigma_{g}
$$

where $\left|\nabla^{\partial} f\right|_{g}^{2}$ is the tangential part of $|\nabla f|_{g}^{2}$. If we write

$$
h_{i j}=g\left(\frac{\partial}{\partial \theta_{i}}, \frac{\partial}{\partial \theta_{j}}\right) \text {, }
$$


then

$$
\left|\nabla^{\partial} f\right|_{g}^{2}=\frac{1}{r^{2}} h^{i j} \frac{\partial f}{\partial \theta_{i}} \cdot \frac{\partial f}{\partial \theta_{j}}=(1+O(\sigma))\left(|\nabla f|^{2}-\left|\frac{\partial f}{\partial r}\right|^{2}\right) .
$$

Thus,

$$
I\left(\sigma, Q_{\sigma}\right) \leq C_{0} \sigma^{2}(1+O(\sigma)) \int_{\partial \mathbf{B}(\sigma)}\left(|\nabla f|^{2}-\left|\frac{\partial f}{\partial r}\right|^{2}\right) d \Sigma_{g}
$$

Therefore,

$$
\begin{aligned}
& E^{2}(\sigma) \leq I\left(\sigma, Q_{\sigma}\right)\left(\left(\int_{\partial \mathbf{B}(\sigma)}\left|\frac{\partial f}{\partial r}\right|^{2} d \Sigma_{g}\right)^{\frac{1}{2}}+c \sigma E^{\prime}(\sigma)^{\frac{1}{2}}\right)^{2} \text { by }(3.12) \\
& \leq 2 I\left(\sigma, Q_{\sigma}\right)\left(\int_{\partial \mathbf{B}(\sigma)}\left|\frac{\partial f}{\partial r}\right|^{2} d \Sigma_{g}+c^{2} \sigma^{2} E^{\prime}(\sigma)\right) \\
& \leq C \sigma^{2}\left((1+O(\sigma))\left(E^{\prime}(\sigma)-\int_{\partial \mathbf{B}(\sigma)}\left|\frac{\partial f}{\partial r}\right|^{2} d \Sigma_{g}\right)\right) \text { by (3.16) } \\
& \times\left(\int_{\partial \mathbf{B}(\sigma)}\left|\frac{\partial f}{\partial r}\right|^{2} d \Sigma_{g}+c^{2} \sigma^{2} E^{\prime}(\sigma)\right) \\
& \leq C \sigma\left((n-2+O(\sigma)) E(\sigma)+\sigma(1+O(\sigma)) \int_{\partial \mathbf{B}(\sigma)}\left|\frac{\partial f}{\partial r}\right|^{2} d \Sigma_{g}\right) \text { by (3.8) } \\
& \times\left((1+O(\sigma)) \int_{\partial \mathbf{B}(\sigma)}\left|\frac{\partial f}{\partial r}\right|^{2} d \Sigma_{g}+c^{2} \sigma(n-2+O(\sigma)) E(\sigma)\right) \\
& \leq C^{\prime}\left(\sigma^{2} E^{2}(\sigma)+\sigma E(\sigma) \int_{\partial \mathbf{B}(\sigma)}\left|\frac{\partial f}{\partial r}\right|^{2} d \Sigma_{g}+\sigma^{2}\left(\int_{\partial \mathbf{B}(\sigma)}\left|\frac{\partial f}{\partial r}\right|^{2} d \Sigma_{g}\right)^{2}\right) \\
& \leq C^{\prime}\left(\left(\sigma^{2}+\epsilon\right) E^{2}(\sigma)+\left(\frac{4}{\epsilon}+1\right) \sigma^{2}\left(\int_{\partial \mathbf{B}(\sigma)}\left|\frac{\partial f}{\partial r}\right|^{2} d \Sigma_{g}\right)^{2}\right)
\end{aligned}
$$

Note that the constant $C$ and $C^{\prime}$ depend only on the Lipschitz constant of $g$ and the constant coming from the Poincaré inequality which only depends on the ellipticity constant of $g$ and the number of wedges of $\mathbf{B}(r)$. Thus, the constants below also depend only on these quantities. By choosing $\sigma>0$ sufficiently small (depending on $C^{\prime}$ ), we see that there exists a constant $K$ 
so that

$$
E(\sigma) \leq K \sigma \int_{\partial \mathbf{B}(\sigma)}\left|\frac{\partial f}{\partial r}\right|^{2} d \Sigma_{g}
$$

Using (3.8), we also have

$$
\begin{aligned}
\sigma E^{\prime}(\sigma) & =(n-2+O(\sigma)) E(\sigma)+\left(2 \sigma+O\left(\sigma^{2}\right)\right) \int_{\partial \mathbf{B}(\sigma)}\left|\frac{\partial f}{\partial r}\right|^{2} d \Sigma_{g} \\
& \geq(n-2+O(\sigma)) E(\sigma)+\frac{2+O(\sigma)}{K} E(\sigma) \\
& =\left(n-2+\frac{2}{K}+O(\sigma)\right) E(\sigma) \\
& \geq(n-2+2 \gamma) E(\sigma)
\end{aligned}
$$

for $\gamma, \sigma>0$ sufficiently small. This implies

$$
\frac{d}{d \sigma}\left(\log \frac{E(\sigma)}{\sigma^{n-2+2 \gamma}}\right) \geq 0
$$

for $\sigma>0$ sufficiently small.

Lemma 3.6. For sufficiently small $\sigma>0$ depending on the Lipschitz bound of $g$ and $\mathbf{B}$ and for any map $f: \mathbf{B}(r) \rightarrow(Y, d)$, we have

$$
\left|\frac{I^{\prime}(\sigma)}{I(\sigma)}-\frac{n-1}{\sigma}-\frac{1}{I(\sigma)} \int_{\partial \mathbf{B}(\sigma)} \frac{\partial}{\partial r} d^{2}(f, Q) d \Sigma_{g}\right| \leq c_{2}
$$

for some constant $c_{2}$ depending on $\mathbf{B}(r)$ and the Lipschitz bound of $g$.

Proof. Let $\left\{\frac{\partial}{\partial r}, \frac{\partial}{\partial \theta_{1}}, \ldots, \frac{\partial}{\partial \theta_{n-1}}\right\}$ be the tangent basis corresponding to the polar coordinates $\left(r, \theta_{1}, \ldots, \theta_{n-1}\right)$ on $W$. We define

$$
v(r, \theta)=\frac{1}{r^{n-1}} \sqrt{\left|\operatorname{det}\left(g\left(\frac{\partial}{\partial \theta^{i}}, \frac{\partial}{\partial \theta^{j}}\right)\right)\right|} .
$$

By the fact that $g_{i j}(0)=\delta_{i j}$, we have

$$
|v(\sigma, \theta)-1|=\left|v(\sigma, \theta)-\lim _{r \rightarrow 0} v(r, \theta)\right| \leq c^{\prime} \sigma
$$

for some constant $c^{\prime}$ depending on the Lipschitz bound of $g$. Since the measure induced on $\partial \mathbf{B}(\sigma)$ by $g$ can be written $d \Sigma_{g}=\sigma^{n-1} v(\sigma, \theta) d \theta$ where $d \theta$ 
is volume form on the standard $(n-1)$-sphere, we have

$$
\frac{\partial}{\partial \sigma} d \Sigma_{g}=(n-1) \sigma^{n-2} v(\sigma, \theta) d \theta+\sigma^{(n-1)} \frac{\partial v}{\partial \sigma}(\sigma, \theta) d \theta
$$

and

$$
d \Sigma_{g} \geq \sigma^{n-1}\left(1-c^{\prime} \sigma\right) d \theta \geq \frac{1}{2} \sigma^{n-1} d \theta
$$

for sufficiently small $\sigma>0$. Thus,

$$
\begin{aligned}
\frac{d}{d \sigma} & \int_{\partial \mathbf{B}(\sigma)} d^{2}(f, Q) d \Sigma_{g} \\
= & \int_{\partial \mathbf{B}(\sigma)} \frac{\partial}{\partial r}\left(d^{2}(f, Q)\right) d \Sigma_{g}+\frac{n-1}{\sigma} \int_{\partial \mathbf{B}(\sigma)} d^{2}(f, Q) d \Sigma_{g} \\
& +\int_{\partial \mathbf{B}(1)} d^{2}(f, Q) \sigma^{n-1} \frac{\partial v}{\partial r}(\sigma, \theta) d \theta
\end{aligned}
$$

which in turn implies

$$
\begin{aligned}
& \left|\frac{I^{\prime}(\sigma)}{I(\sigma)}-\frac{n-1}{\sigma}-\frac{1}{I(\sigma)} \int_{\partial \mathbf{B}(\sigma)} \frac{\partial}{\partial r}\left(d^{2}(f, Q)\right) d \Sigma_{g}\right| \\
& \quad=\frac{1}{I(\sigma)}\left|\int_{\partial \mathbf{B}(1)} d^{2}(f, Q) \sigma^{n-1} \frac{\partial v}{\partial r}(\sigma, \theta) d \theta\right| \\
& \quad \leq \frac{c}{I(\sigma)} \int_{\partial \mathbf{B}(1)} d^{2}(f, Q) \sigma^{n-1} d \theta \\
& \quad \leq \frac{2 c}{I(\sigma)} \int_{\partial \mathbf{B}(\sigma)} d^{2}(f, Q) d \Sigma_{g}=2 c
\end{aligned}
$$

for sufficiently small $\sigma$. This immediately implies (3.17).

Let $f:(\mathbf{B}(r), g) \rightarrow(Y, d)$ be a harmonic map. Inequality (3.17) implies that there exists $\sigma_{0}$ sufficiently small so that for $\sigma<\sigma_{0}$,

$$
\frac{I^{\prime}(\sigma)}{I(\sigma)} \leq \frac{n-1}{\sigma}+\frac{1}{I(\sigma)} \int_{\partial \mathbf{B}(\sigma)} \frac{\partial}{\partial r} d^{2}(f, Q) d \Sigma_{g}+c_{2}
$$

Together, (3.9), (3.14) and (3.19) imply

$$
(1+3 c \sigma) \frac{E^{\prime}(\sigma)}{E(\sigma)}-\frac{I^{\prime}(\sigma)}{I(\sigma)}+\frac{1}{\sigma}+c_{3} \geq 0,
$$

where $c_{3}=c_{1}+c_{2}$. We use this inequality to prove a modified monotonicity which we describe below. For notational simplicity by rescaling the metric 
$g$, we may assume that $3 c \leq 1$ and $\sigma_{0}=1$. Let

$$
J(\sigma)=\max _{s \in[0, \sigma]} I(s)
$$

and set

$$
A=\left\{\sigma: \frac{E^{\prime}(\sigma)}{E(\sigma)}-\frac{J^{\prime}(\sigma)}{J(\sigma)}+\frac{1}{\sigma}+c_{3} \leq 0\right\} .
$$

Roughly speaking, $A$ is the bad set where the ordinary monotonicity formula fails. Note that if $J^{\prime}(s) \neq 0$ then $I(s)$ is increasing and hence $I(s)=J(s)$, $I^{\prime}(s)=J^{\prime}(s)$. If $J^{\prime}(s)=0$, then $I^{\prime}(s) \leq 0$ (for, if $I^{\prime}(s)>0$ then $J$ would be strictly increasing near $s)$. Therefore, we obtain the following pair of inequalities:

$$
\begin{aligned}
\frac{E^{\prime}(\sigma)}{E(\sigma)}-\frac{I^{\prime}(\sigma)}{I(\sigma)}+\frac{1}{\sigma}+c_{3} \geq 0 & \text { for } \sigma \notin A, \\
(1+\sigma) \frac{E^{\prime}(\sigma)}{E(\sigma)}-\frac{I^{\prime}(\sigma)}{I(\sigma)}+\frac{1}{\sigma}+c_{3} \geq 0 & \text { for } \sigma \in A .
\end{aligned}
$$

For $\sigma \in(0,1)$, set

$$
F(\sigma)=E(\sigma) \exp \left(-\int_{A \cap(\sigma, 1)} s \frac{E^{\prime}(s)}{E(s)} d s\right)
$$

Lemma 3.7. For $F(\sigma)$ defined above,

$$
\frac{F^{\prime}(\sigma)}{F(\sigma)}= \begin{cases}\frac{E^{\prime}(\sigma)}{E(\sigma)} & \text { for } \sigma \notin A \\ (1+\sigma) \frac{E^{\prime}(\sigma)}{E(\sigma)} & \text { for } \sigma \in A\end{cases}
$$

Consequently,

$$
\sigma \mapsto e^{c_{3} \sigma} \frac{\sigma F(\sigma)}{I(\sigma)}
$$

is non-decreasing for $\sigma$ sufficiently small. Furthermore, for $Q_{\sigma}$ as in (3.4)

$$
\sigma \mapsto e^{c_{3} \sigma} \frac{\sigma F(\sigma)}{I\left(\sigma, Q_{\sigma}\right)}
$$

is also non-decreasing for $\sigma$ sufficiently small. 
Remark. If $g$ is the Euclidean metric $\delta$, then $c=0$ above and

$$
\sigma \mapsto \frac{\sigma E(\sigma)}{I(\sigma)}
$$

is non-decreasing.

Proof. We first note that as it is an integral of an $L^{1}$ function $\sigma \mapsto E(\sigma)$ is absolutely continuous on $[0, r]$. Let

$$
\varphi(\sigma)=-\int_{A \cap(\sigma, 1)} s \frac{E^{\prime}(s)}{E(s)} d s .
$$

Then, for $a \leq \sigma<\sigma^{\prime} \leq b$, we have

$$
\begin{aligned}
& \left|\varphi(\sigma)-\varphi\left(\sigma^{\prime}\right)\right| \\
& \quad \leq\left|\int_{A \cap\left(\sigma, \sigma^{\prime}\right)} s \frac{E^{\prime}(s)}{E(s)} d s\right| \leq \frac{b}{E(a)}\left|\int_{\sigma}^{\sigma^{\prime}} E^{\prime}(s) d s\right|=\frac{b}{E(a)}\left|E\left(\sigma^{\prime}\right)-E(\sigma)\right|,
\end{aligned}
$$

which implies that the function $\varphi(\sigma)$ and hence also $F(\sigma)$ is absolutely continuous. Here we are assuming that $f$ is non-constant hence $E(a) \neq 0$. Thus,

$$
\varphi^{\prime}(\sigma)=\lim _{\epsilon \rightarrow 0} \frac{\varphi(\sigma+\epsilon)-\varphi(\sigma-\epsilon)}{2 \epsilon}=\lim _{\epsilon \rightarrow 0} \frac{1}{2 \epsilon} \int_{A \cap[\sigma-\epsilon, \sigma+\epsilon]}-s \frac{E^{\prime}(s)}{E(s)} d s .
$$

Therefore $\varphi^{\prime}(\sigma)=0$ for a.e. $\sigma \notin A$ and $\varphi^{\prime}(\sigma)=-\sigma \frac{E^{\prime}(\sigma)}{E(\sigma)}$ for a.e. $\sigma \in A$. This implies (3.23). Finally, note that the function $\sigma \mapsto \log \left(\mathrm{e}^{c_{3} \sigma} \frac{\sigma F(\sigma)}{I(\sigma)}\right)$ is absolutely continuous on any interval $[a, b] \subset(0, r)$. Hence by combining $(3.21-$ 3.23 ), we obtain

$$
\frac{d}{d s} \log \frac{\sigma F(\sigma)}{I(\sigma)} \geq \frac{F^{\prime}(\sigma)}{F(\sigma)}-\frac{I^{\prime}(\sigma)}{I(\sigma)}+\frac{1}{\sigma} \geq-c_{3},
$$

which implies the monotonicity of $\sigma \mapsto \mathrm{e}^{c_{3} \sigma} \frac{\sigma F(\sigma)}{I(\sigma)}$. Furthermore, since $I\left(\sigma_{2}, Q_{\sigma_{1}}\right) \geq I\left(\sigma_{2}, Q_{\sigma_{2}}\right)$, we have for $\sigma_{1}<\sigma_{2}$,

$$
\mathrm{e}^{c_{3} \sigma_{1}} \frac{\sigma_{1} F\left(\sigma_{1}\right)}{I\left(\sigma_{1}, Q_{\sigma_{1}}\right)} \leq \mathrm{e}^{c_{3} \sigma_{2}} \frac{\sigma_{2} F\left(\sigma_{2}\right)}{I\left(\sigma_{2}, Q_{\sigma_{1}}\right)} \leq \mathrm{e}^{c_{3} \sigma_{2}} \frac{\sigma_{2} F\left(\sigma_{2}\right)}{I\left(\sigma_{2}, Q_{\sigma_{2}}\right)} .
$$

This implies the monotonicity of $\sigma \mapsto \mathrm{e}^{c_{3} \sigma} \frac{\sigma F(\sigma)}{I\left(\sigma, Q_{\sigma}\right)}$. 
Lemma 3.7 shows the monotonicity involving a corrected energy term $F(\sigma)$. We now want to show that the correction factor is well-behaved as $\sigma \rightarrow 0$.

\section{Lemma 3.8.}

$$
\int_{A \cap(0,1)} s \frac{E^{\prime}(s)}{E(s)} d s:=\lim _{\sigma \rightarrow 0^{+}} \int_{A \cap(\sigma, 1)} s \frac{E^{\prime}(s)}{E(s)} d s<\infty .
$$

Proof. For $s \in A$,

$$
s \frac{E^{\prime}(s)}{E(s)} \leq s \frac{J^{\prime}(s)}{J(s)}-1-c_{3} s \leq s \frac{J^{\prime}(s)}{J(s)} .
$$

by the definition of the set $A$. Thus, it is sufficient to prove

$$
\lim _{\sigma \rightarrow 0^{+}} \int_{A \cap(\sigma, 1)} s \frac{J^{\prime}(s)}{J(s)} d s<\infty .
$$

We follow the argument of Proposition 3.1 in [14]. Let $M$ be sufficiently large so that for $\sigma \in(0,1]$,

$$
\begin{gathered}
\int_{\mathbf{B}(\sigma)}|\nabla f|^{2} d \mu_{g} \leq M E(\sigma), \\
N:=\mathrm{e}^{c_{3}} \frac{F(1)}{J(1)} \geq \mathrm{e}^{c_{3} \sigma} \frac{\sigma F(\sigma)}{J(\sigma)} \geq \frac{\sigma F(\sigma)}{J(\sigma)}
\end{gathered}
$$

and $K=M N$. Furthermore, let $0<\theta_{1}<\theta_{2} \leq 1$ and $r_{0} \in\left(\theta_{1}, \theta_{2}\right]$. For $s \in$ $\left(\theta_{1}, r_{0}\right)$, we have by $(3.17)$ that

$$
\begin{aligned}
I^{\prime}(s) & \leq \int_{\partial \mathbf{B}(s)} 2 d(f, P) \frac{\partial}{\partial r} d(f, P) d \Sigma_{g}+\frac{n-1+c_{2} s}{s} I(s) \\
& \leq \int_{\partial \mathbf{B}(s)}\left(\frac{1}{\epsilon} d^{2}(f, P)+\epsilon\left(\frac{\partial}{\partial r} d(f, P)\right)^{2}\right) d \Sigma_{g}+\frac{n-1+c_{2} s}{\theta_{1}} I(s) \\
& \leq \int_{\partial \mathbf{B}(s)}\left(\frac{1}{\epsilon} d^{2}(f, P)+\epsilon|\nabla f|^{2}\right) d \Sigma_{g}+\frac{n-1+c_{2} s}{\theta_{1}} I(s) \\
& \leq \epsilon \int_{\partial \mathbf{B}(s)}|\nabla f|^{2} d \Sigma_{g}+\left(\frac{1}{\epsilon}+\frac{C}{\theta_{1}}\right) I(s)
\end{aligned}
$$

for some sufficiently large $C$. Therefore,

$$
I\left(r_{0}\right)-I\left(\theta_{1}\right)=\int_{\theta_{1}}^{r_{0}} I^{\prime}(s) d s \leq \epsilon M E\left(r_{0}\right)+\left(\frac{1}{\epsilon}+\frac{C}{\theta_{1}}\right) \int_{\theta_{1}}^{r_{0}} I(s) d s
$$


Hence

$$
I\left(r_{0}\right)-\epsilon M E\left(r_{0}\right) \leq I\left(\theta_{1}\right)+\left(\frac{1}{\epsilon}+\frac{C}{\theta_{1}}\right)\left(r_{0}-\theta_{1}\right) \max _{s \in\left[\theta_{1}, r_{0}\right]} I(s) .
$$

Since $r_{0} \in\left(\theta_{1}, \theta_{2}\right]$ is arbitrary,

$$
\max _{s \in\left[\theta_{1}, \theta_{2}\right]} I(s)-\epsilon M E\left(\theta_{2}\right) \leq I\left(\theta_{1}\right)+\left(\frac{1}{\epsilon}+\frac{C}{\theta_{1}}\right)\left(\theta_{2}-\theta_{1}\right) \max _{s \in\left[\theta_{1}, \theta_{2}\right]} I(s),
$$

which then implies

$$
\left[1-\left(\frac{1}{\epsilon}+\frac{C}{\theta_{1}}\right)\left(\theta_{2}-\theta_{1}\right)\right] \max _{s \in\left[\theta_{1}, \theta_{2}\right]} I(s)-\epsilon M E\left(\theta_{2}\right) \leq I\left(\theta_{1}\right) .
$$

If $\max _{s \in\left[0, \theta_{1}\right]} I(s) \geq \max _{s \in\left[\theta_{1}, \theta_{2}\right]} I(s)$ then $J(\sigma)$ is identically equal to a constant in $\left[\theta_{1}, \theta_{2}\right]$. If $\max _{s \in\left[0, \theta_{1}\right]} I(s) \leq \max _{s \in\left[\theta_{1}, \theta_{2}\right]} I(s)$, then $\max _{s \in\left[\theta_{1}, \theta_{2}\right]} J(s)=$ $\max _{s \in\left[\theta_{1}, \theta_{2}\right]} I(s)$. Either way, we have

$$
\left[1-\left(\frac{1}{\epsilon}+\frac{C}{\theta_{1}}\right)\left(\theta_{2}-\theta_{1}\right)\right] \max _{s \in\left[\theta_{1}, \theta_{2}\right]} J(s)-\epsilon M E\left(\theta_{2}\right) \leq J\left(\theta_{1}\right),
$$

which immediately implies

$$
\left[1-\left(\frac{1}{\epsilon}+\frac{C}{\theta_{1}}\right)\left(\theta_{2}-\theta_{1}\right)\right] J\left(\theta_{2}\right)-\epsilon M E\left(\theta_{2}\right) \leq J\left(\theta_{1}\right) .
$$

For $\theta_{0} \in(0,1)$ to be determined later, we set

$$
\epsilon=\frac{\theta_{0}^{\frac{j\left(1-\theta_{0}^{n}\right)}{1-\theta_{0}}+n}}{2 K}
$$

to obtain

$$
\begin{gathered}
{\left[1-\left(2 K \theta_{0}^{\frac{-j\left(1-\theta_{0}^{n}\right)}{1-\theta_{0}}-n}+\frac{C}{\theta_{1}}\right)\left(\theta_{2}-\theta_{1}\right)\right] J\left(\theta_{2}\right)} \\
-\frac{E\left(\theta_{2}\right)}{2 N} \theta_{0}^{\frac{j\left(1-\theta_{0}^{n}\right)}{1-\theta_{0}}+n} \leq J\left(\theta_{1}\right)
\end{gathered}
$$

Let

$$
\phi(\theta, n, j)=\frac{1}{2}-\left(2 K \theta^{\frac{-j\left(1-\theta^{n}\right)}{1-\theta}}+\frac{C}{\theta}\right)(1-\theta) .
$$


Then

$$
\lim _{\theta \rightarrow 1} \phi(\theta, n, j)=\frac{1}{2}
$$

uniformly independently of $j, n$. Therefore, there exists $\theta_{0}$ sufficiently close to 1 so that $\phi\left(\theta_{0}, n, j\right)>\frac{1}{4}$ independently of $n$ and $j$. Choose $j$ so that $\theta_{0}^{j}<\frac{1}{4}$. Then

$$
\frac{1}{2}-\left(2 K \theta_{0}^{\frac{-j\left(1-\theta_{0}^{2 n}\right)}{1-\theta_{0}}}+\frac{C}{\theta_{0}}\right)\left(1-\theta_{0}\right)>\theta_{0}^{j}
$$

for any $n$.

Since $F(1)=E(1)$ by definition, we have that

$$
\frac{E(1)}{N}=\frac{J(1)}{\mathrm{e}^{c_{3}}} \leq J(1) .
$$

Thus, (3.27) with $n=0, \theta_{1}=\theta_{0}$ and $\theta_{2}=1$ implies

$$
\left[\frac{1}{2}-\left(2 K \theta_{0}^{\frac{-j}{1-\theta_{0}}}+\frac{C}{\theta_{0}}\right)\left(1-\theta_{0}\right)\right] J(1) \leq J\left(\theta_{0}\right)
$$

and by inequality $(3.28), \theta_{0}^{j} J(1)<J\left(\theta_{0}\right)$. Now suppose $\theta_{0}^{j} J\left(\theta_{0}^{k}\right)<J\left(\theta_{0}^{k+1}\right)$ for $k=0, \ldots, n-1$. Then

$$
\begin{aligned}
\int_{\theta_{0}^{n}}^{1} s \frac{d}{d s} \log J(s) d s & =\sum_{k=0}^{n-1} \int_{\theta_{0}^{k+1}}^{\theta_{0}^{k}} s \frac{d}{d s} \log J(s) d s \\
& \leq \sum_{k=0}^{n-1} \theta_{0}^{k} \int_{\theta_{0}^{k+1}}^{\theta_{0}^{k}} \frac{d}{d s} \log J(s) d s \\
& \leq \sum_{k=0}^{n-1} \theta_{0}^{k} \log \frac{J\left(\theta_{0}^{k}\right)}{J\left(\theta_{0}^{k+1}\right)} \\
& \leq \sum_{k=0}^{n-1} \theta_{0}^{k} \log \theta_{0}^{-j} \quad \text { (by the inductive hypothesis) } \\
& =\sum_{k=0}^{n-1} \log \theta_{0}^{-j \theta_{0}^{k}} \\
& =\log \theta_{0}^{-j \sum_{k=0}^{n-1} \theta_{0}^{k}} \\
& =\log \theta_{0}^{\frac{-j\left(1-\theta_{0}^{n}\right)}{1-\theta_{0}}}
\end{aligned}
$$


Using the fact that $\frac{J^{\prime}(s)}{J(s)} \geq 0$, we obtain

$$
\begin{aligned}
\log \frac{F(1)}{F\left(\theta_{0}^{n}\right)} & =\log \frac{E(1)}{E\left(\theta_{0}^{n}\right)}+\int_{A \cap\left(\theta_{0}^{n}, 1\right)} s \frac{E^{\prime}(s)}{E(s)} d s \\
& \leq \log \frac{E(1)}{E\left(\theta_{0}^{n}\right)}+\int_{A \cap\left(\theta_{0}^{n}, 1\right)} s \frac{J^{\prime}(s)}{J(s)} d s \quad \text { (by (3.24)) } \\
& \leq \log \frac{E(1)}{E\left(\theta_{0}^{n}\right)}+\int_{\theta_{0}^{n}}^{1} s \frac{J^{\prime}(s)}{J(s)} d s \\
& \leq \log \frac{E(1)}{E\left(\theta_{0}^{n}\right)}+\log \theta_{0}^{\frac{-j\left(1-\theta_{0}^{n}\right)}{1-\theta_{0}}} .
\end{aligned}
$$

Therefore, using the fact that $E(1)=F(1)$ and the definition of $N$, we obtain

$$
\theta_{0}^{\frac{j\left(1-\theta_{0}^{n}\right)}{1-\theta_{0}}} E\left(\theta_{0}^{n}\right) \leq F\left(\theta_{0}^{n}\right) \leq \frac{N J\left(\theta_{0}^{n}\right)}{\theta_{0}^{n}}
$$

Thus, we can use the inequality

$$
\frac{E\left(\theta_{0}^{n}\right)}{2 N} \theta_{0}^{\frac{j\left(1-\theta_{0}^{n}\right)}{1-\theta_{0}}+n} \leq \frac{J\left(\theta_{0}^{n}\right)}{2}
$$

in (3.27) with $\theta_{1}=\theta_{0}^{n+1}$ and $\theta_{2}=\theta_{0}^{n}$ to obtain

$$
\left[\frac{1}{2}-\left(2 K \theta_{0}^{\frac{-j\left(1-\theta_{0}^{2 n}\right)}{1-\theta_{0}}}+\frac{C}{\theta_{0}}\right)\left(1-\theta_{0}\right) \cdot\right] J\left(\theta_{0}^{n}\right) \leq J\left(\theta_{0}^{n+1}\right)
$$

Hence, by inequality (3.28), we have

$$
\theta_{0}^{j} J\left(\theta_{0}^{n}\right)<J\left(\theta_{0}^{n+1}\right) .
$$

By induction, inequality (3.29) holds for all $n$ which in turn implies that

$$
\int_{\theta_{0}^{n}}^{1} s \frac{d}{d s} \log J(s) d s \leq \log \theta_{0}^{\frac{-j\left(1-\theta_{0}^{n}\right)}{1-\theta_{0}}}
$$

holds for all $n$. Letting $n \rightarrow \infty$, we obtain

$$
\int_{A \cap(0,1)} s \frac{d}{d s} \log J(s) d s \leq \int_{0}^{1} s \frac{d}{d s} \log J(s) d s \leq \log \theta_{0}^{\frac{-j}{1-\theta_{0}}}<\infty .
$$

This proves (3.25) and the proof is complete. 


\section{Corollary 3.1.}

$$
\alpha:=\lim _{\sigma \rightarrow 0} \frac{\sigma E(\sigma)}{I\left(\sigma, Q_{\sigma}\right)}<\infty
$$

exists.

Proof. By Lemmas 3.7 and 3.8

$$
\begin{aligned}
\lim _{\sigma \rightarrow 0} \frac{\sigma E(\sigma)}{I\left(\sigma, Q_{\sigma}\right)} & =\lim _{\sigma \rightarrow 0}\left(\frac{\sigma F(\sigma)}{I\left(\sigma, Q_{\sigma}\right)} \cdot \frac{E(\sigma)}{F(\sigma)}\right) \\
& =\lim _{\sigma \rightarrow 0} \mathrm{e}^{c_{3} \sigma} \frac{\sigma F(\sigma)}{I\left(\sigma, Q_{\sigma}\right)} \lim _{\sigma \rightarrow 0} \exp \left(\int_{A \cap(\sigma, 1)} s \frac{E^{\prime}(s)}{E(s)} d s\right)<\infty .
\end{aligned}
$$

Definition 3.1. We call $\alpha$ of Corollary 3.1 the order of $f$ at 0 denoted by $\alpha=\operatorname{ord}^{f}(0)$.

Lemma 3.9. Let $\alpha=\operatorname{ord}^{f}(0)$. There exist constants $c_{0}$ and $\sigma_{0}$ depending only on $\mathbf{B}(r)$, the Lipschitz bound and the ellipticity constant of $g$ so that if

$$
\tilde{E}(\sigma):=E(\sigma) \exp \left(c_{0} \int_{A \cap(0, \sigma)} s \frac{E^{\prime}(s)}{E(s)} d s\right),
$$

then

$$
\sigma \mapsto \mathrm{e}^{c_{0} \sigma} \frac{\tilde{E}(\sigma)}{\sigma^{2 \alpha+n-2}}
$$

is non-decreasing for $\sigma \in\left(0, \sigma_{0}\right)$.

Proof. Set

$$
G(\sigma)=E(\sigma) \exp \left(\int_{A \cap(0, \sigma)} s \frac{E^{\prime}(s)}{E(s)} d s\right) .
$$

Since

$$
G(\sigma)=F(\sigma) \exp \left(\int_{A \cap(0,1)} s \frac{E^{\prime}(s)}{E(s)} d s\right),
$$

Lemma 3.7 implies that

$$
\sigma \mapsto \mathrm{e}^{c_{3} \sigma} \frac{\sigma G(\sigma)}{I\left(\sigma, Q_{\sigma}\right)}
$$


is non-decreasing. Since

$$
\lim _{\sigma \rightarrow 0} \frac{E(\sigma)}{G(\sigma)}=\lim _{\sigma \rightarrow 0} \exp \left(-\int_{A \cap(0, \sigma)} s \frac{E^{\prime}(s)}{E(s)} d s\right) \leq 1,
$$

we have

$$
\alpha=\lim _{\sigma \rightarrow 0} \frac{\sigma E(\sigma)}{I\left(\sigma, Q_{\sigma}\right)} \leq \lim _{\sigma \rightarrow 0} \frac{\sigma G(\sigma)}{I\left(\sigma, Q_{\sigma}\right)}=\lim _{\sigma \rightarrow 0} \mathrm{e}^{c_{3} \sigma} \frac{\sigma G(\sigma)}{I\left(\sigma, Q_{\sigma}\right)} .
$$

Therefore, we obtain that

$$
\alpha \leq \mathrm{e}^{c_{3} \sigma} \frac{\sigma G(\sigma)}{I\left(\sigma, Q_{\sigma}\right)}=\mathrm{e}^{c_{3} \sigma} \frac{\sigma E(\sigma)}{I\left(\sigma, Q_{\sigma}\right)} \exp \left(\int_{A \cap(0, \sigma)} s \frac{E^{\prime}(s)}{E(s)} d s\right)
$$

and

$$
\frac{\sigma E(\sigma)}{I\left(\sigma, Q_{\sigma}\right)} \leq \mathrm{e}^{c_{3} \sigma} \frac{\sigma G(\sigma)}{I\left(\sigma, Q_{\sigma}\right)} \leq \mathrm{e}^{\sigma_{3}} \frac{G(1)}{I(1)}=: K
$$

Now by the proof of Lemma 3.8, if $\theta_{0}^{n+1} \leq \sigma<\theta_{0}^{n}$, then

$$
\begin{aligned}
& \int_{A \cap(0, \sigma)} s \frac{E^{\prime}(s)}{E(s)} d s \leq \int_{0}^{\sigma} s \frac{J^{\prime}(s)}{J(s)} d s \\
& \leq \int_{0}^{\theta_{0}^{n}} s \frac{J^{\prime}(s)}{J(s)} d s \\
& \leq \sum_{k=n}^{\infty} \int_{\theta_{0} k+1}^{\theta_{0}^{k}} s \frac{J^{\prime}(s)}{J(s)} d s \\
& \leq \log \theta_{0}^{-j \sum_{k=n}^{\infty} \theta_{0}{ }^{k}} \\
& =\log \theta_{0}{ }^{-\frac{j \theta_{0} n}{1-\theta_{0}}} \\
& \leq c_{4} \theta_{0}^{n} \\
& \leq \frac{c_{4}}{\theta_{0}} \sigma=: c_{5} \sigma \text {. }
\end{aligned}
$$

Thus, this implies that for any $c_{0} \geq 1$ and for

$$
\begin{gathered}
\tilde{E}(\sigma):=E(\sigma) \exp \left(c_{0} \int_{A \cap(0, \sigma)} s \frac{E^{\prime}(s)}{E(s)} d s\right), \\
E(\sigma) \leq \tilde{E}(\sigma) \leq \mathrm{e}^{c_{0} c_{5} \sigma} E(\sigma),
\end{gathered}
$$


and by $(3.30)$

$$
\alpha \leq \mathrm{e}^{\left(c_{3}+c_{5}\right) \sigma} \frac{\sigma E(\sigma)}{I\left(\sigma, Q_{\sigma}\right)} .
$$

Furthermore, (3.13) and (3.17) imply that

$$
\begin{aligned}
2 E(\sigma) & \leq \int_{\partial \mathbf{B}(\sigma)} \frac{\partial}{\partial r} d^{2}(f, Q) d \Sigma_{g}+I(\sigma)+k \sigma^{2} E^{\prime}(\sigma) \\
& \leq I^{\prime}(\sigma)-\frac{n-1}{\sigma} I(\sigma)+\left(1+c_{2}\right) I(\sigma)+k \sigma^{2} E^{\prime}(\sigma),
\end{aligned}
$$

where the constants $c_{2}$ and $k$ depend on the Lipschitz bound of $g$. Inequality (3.35) implies

$$
\begin{aligned}
\frac{\frac{2 \sigma E(\sigma)}{I(\sigma)}+n-1-O(\sigma)}{\sigma} & \leq \frac{I^{\prime}(\sigma)}{I(\sigma)}+k \sigma^{2} \frac{E^{\prime}(\sigma)}{I(\sigma)} \\
& \leq \frac{I^{\prime}(\sigma)}{I(\sigma)}+k K \sigma \frac{E^{\prime}(\sigma)}{E(\sigma)} \\
& \leq \frac{G^{\prime}(\sigma)}{G(\sigma)}+\frac{1}{\sigma}+k K \sigma \frac{E^{\prime}(\sigma)}{E(\sigma)}
\end{aligned}
$$

for any $Q \in Y$. By combining (3.36) with (3.34) and absorbing the exponential terms in $O(\sigma)$ we obtain

$$
\frac{2 \alpha+n-1-O(\sigma)}{\sigma} \leq \frac{G^{\prime}(\sigma)}{G(\sigma)}+\frac{1}{\sigma}+k K \sigma \frac{E^{\prime}(\sigma)}{E(\sigma)}
$$

If $c_{0} \geq k K+1$, then

$$
\frac{\tilde{E}^{\prime}(\sigma)}{\tilde{E}(\sigma)} \geq\left(1+c_{0} \sigma\right) \frac{E^{\prime}(\sigma)}{E(\sigma)} \geq(1+(k K+1) \sigma) \frac{E^{\prime}(\sigma)}{E(\sigma)}=\frac{G^{\prime}(\sigma)}{G(\sigma)}+k K \sigma \frac{E^{\prime}(\sigma)}{E(\sigma)} .
$$

Therefore, using (3.37), we can choose $c_{0}$ sufficiently large so that

$$
\frac{2 \alpha+n-2}{\sigma}-c_{0} \leq \frac{\tilde{E}^{\prime}(\sigma)}{\tilde{E}(\sigma)}
$$

and hence

$$
\sigma \mapsto \mathrm{e}^{c_{0} \sigma} \frac{\tilde{E}(\sigma)}{\sigma^{2 \alpha+n-2}}
$$

is non-decreasing. 
By combining Lemma 3.7 and Corollary 3.9 we obtain

Corollary 3.2. Let $\mathbf{B}$ be a dimension-n, codimension- $\nu$ local model, $g$ a normalized Lipschitz Riemannian metric defined on $\mathbf{B}(r),(Y, d)$ an $N P C$ space, $f:(\mathbf{B}(r), g) \rightarrow Y$ a harmonic map and $\alpha=\operatorname{ord}^{f}(0)$. Then there exist constants $k$ and $\sigma_{0} \leq 1$ depending on $\mathbf{B}(r)$, the Lipschitz bound and the ellipticity constant of $g$ so that

$$
\frac{\sigma E(\sigma)}{I\left(\sigma, Q_{\sigma}\right)} \leq \mathrm{e}^{k \rho} \frac{\rho E(\rho)}{I\left(\rho, Q_{\rho}\right)} \quad \text { for } 0<\sigma \leq \rho \leq \sigma_{0}
$$

and

$$
\frac{E(\sigma)}{\sigma^{2 \alpha+n-2}} \leq \mathrm{e}^{k \rho} \frac{E(\rho)}{\rho^{2 \alpha+n-2}} \quad \text { for } 0<\sigma \leq \rho \leq \sigma_{0}
$$

Proof. Formula (3.38) immediately follows from Lemma 3.7. Furthermore, Lemma 3.9 and (3.33) immediately imply (3.39).

So far in this section, we assumed that our metric $g$ is normalized at 0 (i.e., $\left.g_{i j}(0)=\delta_{i j}\right)$. We will relax this assumption and still show the monotonicity formula for the energy of a harmonic map (cf. Proposition 3.1 below). Let $\mathbf{B}$ be a dimension- $n$, codimension- $(n-k)$ local model and $g$ a Lipschitz metric on $\mathbf{B}(r)$ with ellipticity constant $\lambda \in(0,1]$. For $x \in \mathbf{B}(r)$, recall that $R(x)$ is defined to be the radius of the largest homogeneous ball centered at $x$ contained in $\mathbf{B}(r)$. Assume that $x$ is a codimension- $(n-j)$ singular point. Let $\mathbf{B}^{\prime}$ be a dimension- $n$, codimension- $(n-j)$ local model and $L_{x}: \mathbf{B}^{\prime}(\lambda R(x)) \rightarrow L_{x}\left(\mathbf{B}^{\prime}(\lambda R(x))\right) \subset \mathbf{B}$ be a homeomorphism satisfying properties (i) through (iv) of Proposition 2.1. In particular, recall this implies that $h:=L_{x}^{*} g$ is a normalized metric. If $f: \mathbf{B}(r) \rightarrow Y$ is any finite energy map, then $f \circ L_{x}$ is defined on $\mathbf{B}^{\prime}(\lambda R(x))$. Moreover,

$$
\int_{B_{x}(\sigma)}|\nabla f|^{2} d \mu_{g}=\int_{L_{x}^{-1}\left(B_{x}(\sigma)\right)}\left|\nabla\left(f \circ L_{x}\right)\right|^{2} d \mu_{h}
$$

and

$$
\int_{\mathbf{B}^{\prime}(\sigma)}\left|\nabla\left(f \circ L_{x}\right)\right|^{2} d \mu_{h}=\int_{L_{x}\left(\mathbf{B}^{\prime}(\sigma)\right)}|\nabla f|^{2} d \mu_{g} .
$$

This in turn implies that if $f$ is a harmonic map with respect to the metric $g$, then $f \circ L_{x}$ is a harmonic map with respect to the metric $h$. We call $f \circ L_{x}$ the normalized harmonic map at $x$. Recall that $\sigma_{0}$ was defined above 
as the upper bound for which monotonicity formulae of Lemma 3.5 and Corollary 3.2 are valid for any harmonic map from a local model with a normalized metric. Therefore, these monotonicity formulae for $f \circ L_{x}$ are valid for balls $\mathbf{B}^{\prime}(\sigma)$ contained in $\mathbf{B}^{\prime}\left(r_{0}(x)\right)$ for

$$
r_{0}(x):=\min \left\{\sigma_{0}, \lambda R(x)\right\}
$$

For a harmonic map $f$, we define the order of $f$ at $x$ as

$$
\operatorname{ord}^{f}(x):=\operatorname{ord}^{f \circ L_{x}}(0) \text {. }
$$

We also define $E_{x}(\sigma)$ and $I_{x}(\sigma)$ for $\sigma$ sufficiently small by setting

$$
E_{x}(\sigma)=\int_{B_{x}(\sigma)}|\nabla f|^{2} d \mu_{g}
$$

and

$$
I_{x}(\sigma)=\int_{\partial B_{x}(\sigma)} d^{2}(f, Q) d \Sigma_{g}
$$

Proposition 3.1. Let $\mathbf{B}$ be a dimension- $n$, codimesion- $(n-k)$ local model, $g$ a Lipschitz metric defined on $\mathbf{B}(r)$ with ellipticity constant $\lambda \in(0,1],(Y, d)$ an NPC space and $f:(\mathbf{B}(r), g) \rightarrow Y$ a harmonic map. Then there exist constants $\gamma>0$ and $C \geq 1$ depending on $\mathbf{B}(r)$, the Lipschitz bound and the ellipticity constant of $g$ so that for every $x \in \mathbf{B}(r)$,

$$
\frac{E_{x}(\sigma)}{\sigma^{n-2+2 \gamma}} \leq C \frac{E_{x}(\rho)}{\rho^{n-2+2 \gamma}}, 0<\sigma<\rho \leq r(x)
$$

and

$$
\frac{E_{x}(\sigma)}{\sigma^{n-2+2 \alpha_{x}}} \leq C \frac{E_{x}(\rho)}{\rho^{n-2+2 \alpha_{x}}}, 0<\sigma<\rho \leq r(x),
$$

where

$$
r(x)=\lambda r_{0}(x)=\min \left\{\lambda \sigma_{0}, \lambda^{2} R(x)\right\}
$$

Here, recall that $R(x)$ is the radius of the largest homogeneous ball contained in $\mathrm{S} t(x)$ and $\sigma_{0}>0$ defined in Corollary 3.2 is the number associated with the monotonicity formulae. 
Proof. Let $L_{x}$ be as above and set

$$
\mathcal{E}(\sigma)=\int_{\mathbf{B}^{\prime}(\sigma)}\left|\nabla\left(f \circ L_{x}\right)\right|^{2} d \mu_{h}
$$

Lemma 3.5 and Corollary 3.2 imply that there exists a constant $c \geq 1$ so that

$$
\frac{\mathcal{E}(s)}{s^{n-2+2 \gamma}} \leq c \frac{\mathcal{E}(r)}{r^{n-2+2 \gamma}}, 0<s<r \leq r_{0}(x)
$$

and

$$
\frac{\mathcal{E}(s)}{s^{n-2+2 \alpha_{x}}} \leq c \frac{\mathcal{E}(r)}{r^{n-2+2 \alpha_{x}}}, 0<s<r \leq r_{0}(x)
$$

with $r_{0}(x)$ as in (3.42). Let $\Gamma=n-2+2 \gamma$ or $\Gamma=n-2+2 \alpha_{x}$. Fix $\sigma, \rho$ so that $0<\sigma<\rho \leq r(x)$. Then, since $\lambda \leq 1,0<\lambda^{-1} \sigma, \lambda \rho \leq r_{0}(x)$. We prove (3.43) and (3.44) by considering the following two cases. In the first case, we assume $\lambda^{-1} \sigma \leq \lambda \rho$. We then have

$$
\begin{aligned}
\frac{E_{x}(\sigma)}{\sigma^{\Gamma}} & \leq \frac{\mathcal{E}\left(\lambda^{-1} \sigma\right)}{\sigma^{\Gamma}} \text { by }(3.40) \text { and the fact that } L_{x}^{-1}\left(B_{x}(\sigma)\right) \subset \mathbf{B}^{\prime}\left(\lambda^{-1} \sigma\right) \\
& \leq \frac{1}{\lambda^{\Gamma}} \frac{\mathcal{E}\left(\lambda^{-1} \sigma\right)}{\left(\lambda^{-1} \sigma\right)^{\Gamma}} \\
& \leq \frac{c}{\lambda^{\Gamma}} \frac{\mathcal{E}(\lambda \rho)}{(\lambda \rho)^{\Gamma}} \text { by }(3.46) \text { or }(3.47) \text { and the assumption that } \lambda^{-1} \sigma \leq \lambda \rho \\
& \leq \frac{c}{\lambda^{\Gamma}} \frac{E_{x}(\rho)}{(\lambda \rho)^{\Gamma}} \quad \text { by }(3.41) \text { and the fact that } L_{x}\left(\mathbf{B}^{\prime}(\lambda \rho)\right) \subset B_{x}(\rho) \\
& \leq \frac{c}{\lambda^{2 \Gamma}} \frac{E_{x}(\rho)}{\rho^{\Gamma}} .
\end{aligned}
$$

In the second case, we assume $\lambda^{-1} \sigma>\lambda \rho$. We then have

$$
\begin{aligned}
\frac{E_{x}(\sigma)}{\sigma^{\Gamma}} & \leq \frac{E_{x}(\rho)}{\sigma^{\Gamma}} \quad \text { by the fact that } B_{x}(\sigma) \subset B_{x}(\rho) \\
& \leq \frac{E_{x}(\rho)}{\left(\lambda^{2} \rho\right)^{\Gamma}} \quad \text { by the fact that } \frac{1}{\sigma}<\frac{1}{\lambda^{2} \rho} \\
& \leq \frac{1}{\lambda^{2 \Gamma}} \frac{E_{x}(\rho)}{\rho^{\Gamma}}
\end{aligned}
$$

In either case, we have proven our assertion by setting $C=\frac{c}{\lambda^{2 \Gamma}}$. 


\section{Hölder continuity}

In this section, we prove the Hölder continuity of a harmonic map from a Riemannian complex into an NPC space $(Y, d)$. Such a result in the case when the domain metric is smooth was discussed in $[2,7]$. Using the results of the previous section, we are able to consider a Lipschitz metric $g$. Moreover, we provide the explicit dependence of the Hölder exponent and Hölder constant on $g, E^{f}$ and $\mathbf{B}$. By dependence on $\mathbf{B}$, we mean the dependence on the dimension of $\mathbf{B}$, the number of wedges as well as the wedge angles of $\mathbf{B}$. In the later sections, we give a condition for which the Hölder continuity can be improved to Lipschitz continuity. Our proof follows the approach in $[2,9]$. The main technical difficulty is that monotonicity only works for small balls (cf. Remark 3.3 [4]). Therefore in order to obtain the energy decay estimate for large balls (cf. Proposition 4.3), we need the rather technical inductive process described in Proposition 4.2 and Corollary 4.2. We first prove some results pertaining to the geometry of local models.

Proposition 4.1. Fix integers $k$, $n$ so that $0 \leq k<n$. Assume that the sets $\mathcal{B}_{k}, \ldots, \mathcal{B}_{n}$ have the following properties:

(1) for each $j \in\{k, k+1, \ldots, n\}, \mathcal{B}_{j}$ is a finite set of dimension-n, codimension- $(n-j)$ local models, and

(2) if $\mathbf{B} \in \mathcal{B}_{j}$ for some $j \in\{k, k+1, \ldots, n-1\}$, then for any $x \in \mathbf{B}$ and $\sigma \leq R(x)$, we have $B_{x}(\sigma)$ is isometric to $\mathbf{B}^{\prime}(\sigma)$ where $\mathbf{B}^{\prime} \in \mathcal{B}_{i}$ for some $i \in\{j, j+1, \ldots, n\}$.

Then for all $j \in\{k, \ldots, n\}$ and $\mathbf{B} \in \mathcal{B}_{j}$, there exists $\kappa(\mathbf{B}) \geq 1$ so that for all $i \in\{j, \ldots, n\}$,

$$
\frac{|x-\bar{x}|}{R(x)}<\kappa(\mathbf{B}), \forall x \in S_{i}-S_{i-1} \subset \mathbf{B}, \forall \bar{x} \in \pi_{i-1}(x)
$$

Proof. We first make the following observation. Let $\mathbf{B}$ be any dimension$n$, codimension- $(n-k)$ local model and $x \in \mathbf{B}$. Recall that $D=S_{k}$ is isometric to $\mathbf{R}^{k}$ and hence the closest point projection map $\pi_{D}: \mathbf{B} \rightarrow D$ is well-defined. For any $x \in \mathbf{B}-D$, let $t \mapsto x_{t}$ be the constant speed parameterization of a ray starting from $\pi_{D}(x)$ and going through $x=x_{1}$. Assume $x \in S_{i}-S_{i-1}$ and let $\bar{x} \in \pi_{i-1}(x)$. Since $t \mapsto x_{t}$ and $t \mapsto \bar{x}_{t}$ are rays from 
$\pi_{D}(\bar{x})=\pi_{D}(x)$, we see that $\bar{x}_{t} \in \pi_{i-1}\left(x_{t}\right)$ and $t|x-\bar{x}|=\left|x_{t}-\bar{x}_{t}\right|$. Furthermore, we also see that $t R(x)=R\left(x_{t}\right)$. Thus, we observe that

$$
\frac{|x-\bar{x}|}{R(x)}=\frac{\left|x_{t}-\bar{x}_{t}\right|}{R\left(x_{t}\right)}, \forall t \in(0, \infty)
$$

We now proceed with the proof of the assertion by reverse induction on $j$. First note that there is nothing to prove for $j=n$ since $\mathcal{B}_{n}=\left\{\mathbf{R}^{n}\right\}$. Assume that the assertion is true for $\mathcal{B}_{n}, \mathcal{B}_{n-1}, \ldots, \mathcal{B}_{j+1}$. By (4.1), we only need to show that for each $\mathbf{B} \in \mathcal{B}_{j}$, there exists $\kappa(\mathbf{B})$ so that for any $i \in\{j, \ldots, n\}$,

$$
\frac{|x-\bar{x}|}{R(x)}<\kappa(\mathbf{B}), \forall x \in U \cap\left(S_{i}-S_{i-1}\right) \subset \mathbf{B}, \forall \bar{x} \in \pi_{i-1}(x),
$$

where $U$ is the set of points of $\mathbf{B}$ at a distance 1 from $D$. Suppose this is not true, i.e. for some fixed $i$, there exist $\mathbf{B} \in \mathcal{B}_{j}$ and a sequence $y_{n} \in$ $U \cap\left(S_{i}-S_{i-1}\right)$ so that

$$
\frac{\left|y_{n}-\overline{y_{n}}\right|}{R\left(y_{n}\right)} \rightarrow \infty
$$

with $\overline{y_{n}} \in \pi_{i-1}\left(y_{n}\right)$. Since $U$ is a compact set, we may assume (by choosing a subsequence if necessary) that $y_{n} \rightarrow y$. By the definition of $U, y \in S_{m}-$ $S_{m-1}$ for $m>j$. Let us also assume $i>j+1$. By the facts that $y \in S_{m}-$ $S_{m-1},\left|y_{n}-y\right| \rightarrow 0$ and assumption (2.2), we can assume that $y_{n}$ and $\overline{y_{n}}$ are points in a local model $\mathbf{B}^{\prime} \in \mathcal{B}_{m}$. Since $m>j$ then the inductive hypothesis implies that $\frac{\left|y_{n}-\overline{y_{n}}\right|}{R\left(y_{n}\right)}$ is bounded which contradicts (4.2). Now consider the case $i=j+1$, hence $m=j+1$. Since in this case $\left|y_{n}-\overline{y_{n}}\right|=1$ and because $U \cap S_{j+1}$ is compact and hence $R\left(y_{n}\right) \geq c$ independent of $n$, we also obtain a contradiction to (4.2). This completes the proof.

Corollary 4.1. Let $\mathbf{B}$ be a dimension-n, codimension- $(n-k)$ local model. There exists $\kappa \geq 1$ so that for any $i=\{k, \ldots, n\}$,

$$
\frac{\left|x-\pi_{i-1}(x)\right|}{R(x)}<\kappa, \forall x \in S_{i}-S_{i-1} \subset \mathbf{B} .
$$

Proof. Apply Proposition 4.2 with $\mathcal{B}_{j}$ for $j=k, . ., n$ defined to be the set of model spaces so that $\mathbf{B}^{\prime} \in \mathcal{B}_{j}$ if and only if there exists $x \in S_{j}-S_{j-1}$ so that $B_{x}(R(x))$ is isometric to $\mathbf{B}^{\prime}(R(x))$. We are done by setting $\kappa=\max \kappa(\mathbf{B})$ where the maximum is taken over $\mathbf{B} \in \mathcal{B}_{j}$ and $j=k, \ldots, n$. 
Proposition 4.2. Fix $\lambda \in(0,1]$ and integers $k$, $n$ so that $0 \leq k<n$. Assume that the sets $\mathcal{B}_{k}, \ldots, \mathcal{B}_{n}$ have the following properties:

(1) for each $j \in\{k, k+1, \ldots, n\}, \mathcal{B}_{j}$ is a finite set of dimension-n, codimension- $(n-j)$ local models, and

(2) if $\mathbf{B} \in \mathcal{B}_{j}$ for some $j \in\{k, k+1, \ldots, n-1\}$, then for any $x \in \mathbf{B}$ and $\sigma \leq R(x)$, we have $B_{x}(\sigma)$ is isometric to $\mathbf{B}^{\prime}(\sigma)$ where $\mathbf{B}^{\prime} \in \mathcal{B}_{i}$ for some $i \in\{j, j+1, \ldots, n\}$.

Then, there exists $C \geq 1$ so that for any $\mathbf{B} \in \mathcal{B}_{j}, j \in\{k, k+1, \ldots, n\}$, and $x \in \mathbf{B}$, there exist an ordered sequence

$$
x_{1} \triangleright x_{2} \triangleright \cdots \triangleright x_{m}
$$

of points in $\mathbf{B}$ with $x_{1}=x, x_{m} \in S_{j}$ and positive numbers $\sigma_{1}, \ldots, \sigma_{m-1}$ with the property that

$$
\frac{\sigma_{i}}{R\left(x_{i}\right)} \leq C, \frac{\sigma_{i}}{R\left(x_{i+1}\right)} \leq 1 \quad \text { and } \quad B_{x_{i}}\left(R\left(x_{i}\right)\right) \subset B_{x_{i+1}}\left(\lambda^{2} \sigma_{i}\right)
$$

Proof. We first need some preliminary constructions on each element $\mathbf{B}$ of $\cup_{j=k}^{n} \mathcal{B}_{j}$. So fix $j$ and $\mathbf{B} \in \mathcal{B}_{j}$. Let $U$ be the set of points of $\mathbf{B}$ at a distance 1 from $D=S_{j}$. Set

$$
U_{n}=U, U_{n-1}=\pi_{n-1}\left(U_{n}\right), \ldots, \quad U_{j}=\pi_{j}\left(U_{j+1}\right) .
$$

By the convexity of the faces of $\mathbf{B}$, we see that $U_{j+1} \subset \subset \mathbf{B}-S_{j}$.

For $i=j+1, \ldots, n-1$, we define a positive number $R_{i}$ and a subset $N_{i}$ of $\mathbf{B}$ by an inductive procedure.

- First we define $R_{j+1}$ and $N_{j+1}$.

Let $V_{j+1}$ be so that

$$
U_{j+1} \subset \subset V_{j+1} \subset \subset S_{j+1}-S_{j}
$$

Thus, there exists $R_{j+1}>0$ so that

$$
R\left(x^{\prime}\right) \geq R_{j+1}, \forall x^{\prime} \in V_{j+1} .
$$

We can choose a neighborhood $N_{j+1} \subset \subset \mathbf{B}-S_{j}$ of $U_{j+1}$ so that

$$
B_{x^{\prime}}\left(2 \sigma^{\prime}\right) \subset \mathbf{B}-S_{j}, \forall x^{\prime} \in \Pi_{j+1}(x) \text { where } x \in N_{j+1} \text { and } \sigma^{\prime}=\left|x-x^{\prime}\right|,
$$




$$
\Pi_{j+1}\left(N_{j+1}\right) \subset V_{j+1}
$$

and

$$
U_{j+1} \subset \subset N_{j+1}
$$

- Assuming we have chosen positive numbers $R_{j+1}, \ldots, R_{i-1}$, open sets $N_{j+1} \subset \subset \mathbf{B}-S_{j}, \ldots, N_{i-1} \subset \subset \mathbf{B}-S_{i-2}$ and sets $V_{j+1}, \ldots, V_{i-1}$ so that for $l \in\{j+1, \ldots, i-1\}$,

$$
\begin{gathered}
B_{x^{\prime}}\left(2 \sigma^{\prime}\right) \subset \mathbf{B}-S_{l-1}, \quad \forall x^{\prime} \in \prod_{l}(x) \text { where } x \in N_{l} \text { and } \sigma^{\prime}=\left|x-x^{\prime}\right|, \\
\prod_{l}\left(N_{l}\right) \subset V_{l}, \\
U_{l}-\bigcup_{m=j+1}^{l-1} N_{m} \subset \subset N_{l},
\end{gathered}
$$

and

$$
R\left(x^{\prime}\right) \geq R_{l}, \forall x^{\prime} \in V_{l},
$$

we define $R_{i}$ and $N_{i}$ as follows:

First note that

$$
U_{i}-\bigcup_{m=j+1}^{i-1} N_{m} \subset \subset U_{i}-S_{i-1}
$$

hence, we can choose $V_{i} \subset S_{i}$ be so that

$$
U_{i}-\bigcup_{m=j+1}^{i-1} N_{m} \subset \subset V_{i} \subset \subset U_{i}-S_{i-1}
$$

Thus, there exists $R_{i}>0$ so that

$$
R\left(x^{\prime}\right) \geq R_{i}, \forall x^{\prime} \in V_{i}
$$

We can choose a neighborhood $N_{i} \subset \subset \mathbf{B}-S_{i-1}$ of $U_{i}-\cup_{l=j+1}^{i-1} N_{l}$ so that

$$
\begin{gathered}
B_{x^{\prime}}\left(2 \sigma^{\prime}\right) \subset \mathbf{B}-S_{i-1}, \forall x^{\prime} \in \Pi_{i}(x) \text { where } x \in N_{i} \text { and } \sigma^{\prime}=\left|x-x^{\prime}\right|, \\
\Pi_{i}\left(N_{i}\right) \subset V_{i}
\end{gathered}
$$

and

$$
U_{i}-\bigcup_{l=j+1}^{i-1} N_{l} \subset \subset N_{i}
$$

In summary, we have constructed sets $U=U_{n}, \ldots, U_{j+1}, V_{n}, \ldots, V_{j+1}$, positive numbers $R_{j+1}, \ldots, R_{n-1}$ and open sets $N_{j+1} \subset \subset \mathbf{B}-S_{j}, \ldots$, 
$N_{n-1} \subset \subset \mathbf{B}-S_{n-2}$ so that for each $l=j+1, \ldots, n-1$,

$$
B_{x^{\prime}}\left(2 \sigma^{\prime}\right) \subset \mathbf{B}-S_{l-1}
$$

for $x \in N_{l}, x^{\prime} \in \Pi_{l}(x), \sigma^{\prime}=\left|x-x^{\prime}\right|$,

$$
\Pi_{l}\left(N_{l}\right) \subset V_{l}
$$

and

$$
R\left(x^{\prime}\right) \geq R_{l}, \forall x^{\prime} \in V_{l} .
$$

By assumption 2 , and by shrinking $N_{j+1}, \ldots, N_{n-1}$ if necessary, we assume the following for $x \in N_{l}, x^{\prime} \in \Pi_{l}(x), \sigma^{\prime}=\left|x-x^{\prime}\right|$ :

$$
\text { we can identify } B_{x^{\prime}}\left(\sigma^{\prime}\right) \text { with } \mathbf{B}^{\prime}\left(\sigma^{\prime}\right) \text { where } \mathbf{B}^{\prime} \in \mathcal{B}_{l} \text {. }
$$

Since

$$
N=\bigcup_{l=j+1}^{n-1} N_{l}
$$

covers the singular set of $U$, there exists $R_{n}>0$ so that

$$
R(x) \geq R_{n}, \forall x \in U-N
$$

In the above, for each $j \in\{k, \ldots, n\}$ and $\mathbf{B} \in \mathcal{B}_{j}$, we associated sets

$$
U=U(\mathbf{B}), U_{n-1}=U_{n-1}(\mathbf{B}), \ldots, U_{j+1}=U_{j+1}(\mathbf{B}),
$$

positive numbers

$$
R_{j+1}=R_{j+1}(\mathbf{B}), \ldots, R_{n}=R_{n}(\mathbf{B})
$$

and open sets

$$
N_{j+1}=N_{j+1}(\mathbf{B}), \ldots, N_{n-1}=N_{n-1}(\mathbf{B}), N=N(\mathbf{B}) .
$$

Let

$$
C(\mathbf{B})=\max \left\{\frac{2}{R_{j+1}(\mathbf{B})}, \ldots, \frac{2}{R_{n}(\mathbf{B})}, 1\right\}
$$

and

$$
C=\lambda^{-2} \max \left\{C(\mathbf{B}): \mathbf{B} \in \mathcal{B}_{j}, j=k, \ldots, n\right\}
$$


Furthermore, for $l=k, \ldots, n$, let

$$
\hat{R}_{l}=\min \left\{R_{l}(\mathbf{B}): \mathbf{B} \in \mathcal{B}_{j}, j=k, \ldots, n\right\}
$$

We now proceed with the proof of the proposition. Since $\mathcal{B}_{n}=\left\{\mathbf{R}^{n}\right\}$, there is nothing to prove for $\mathcal{B}_{n}$. We now prove the assertion for $\mathcal{B}_{j}$ for any $j=k, \ldots, n$ by doing a reverse induction; more specifically, assume that the assertion is true for $\mathcal{B}_{n}, \mathcal{B}_{n-1}, \ldots, \mathcal{B}_{j+1}$ and prove the assertion for $\mathcal{B}_{j}$.

Now given $x \in \mathbf{B} \in \mathcal{B}_{j}$, we need to show that there exist an ordered sequence

$$
x_{1} \triangleright x_{2} \triangleright \cdots \triangleright x_{m}
$$

of points in $\mathbf{B}$ with $x_{1}=x, x_{m} \in S_{j}$ and positive numbers $\sigma_{1}, \ldots, \sigma_{m-1}$ satisfying (4.3); i.e.,

$$
\frac{\sigma_{i}}{R\left(x_{i}\right)} \leq C, \frac{\sigma_{i}}{R\left(x_{i+1}\right)} \leq 1 \text { and } B_{x_{i}}\left(R\left(x_{i}\right)\right) \subset B_{x_{i+1}}\left(\lambda^{2} \sigma_{i}\right)
$$

If $x$ is in the lowest dimensional stratum $D=S_{j}$, there is nothing to prove so assume $x \in \mathbf{B}-S_{j}$. By the scale invariance of the assertion, we may assume that $x \in U(\mathbf{B})$. If $x \in U(\mathbf{B})-N(\mathbf{B})$, then let $x_{1}=x, x_{2} \in \Pi_{j}\left(x_{1}\right)$ and $\sigma_{1}=2 \lambda^{-2}$. Since

$$
\frac{\sigma_{1}}{R\left(x_{1}\right)}=\frac{2 \lambda^{-2}}{R\left(x_{1}\right)} \leq \frac{2 \lambda^{-2}}{\hat{R}_{n}} \leq C
$$

$R\left(x_{1}\right) \leq 1$ and $R\left(x_{2}\right)=\infty$, we are done.

So assume $x \in N(\mathbf{B})$; in particular, $x \in N_{l}(\mathbf{B})$ for some $l=j+1, \ldots$, $n-1$. In this case, we use the inductive hypothesis. More specifically, choose $x^{\prime} \in \Pi_{l}(x)$, let $\sigma^{\prime}=\left|x-x^{\prime}\right|$, use (4.4) and note that the inductive hypothesis implies that for any $\mathbf{B}^{\prime} \in \mathcal{B}_{l}$ and any $x \in \mathbf{B}^{\prime}$, there exist a sequence

$$
x_{1}=x \triangleright x_{2} \triangleright \cdots \triangleright x_{m} \in S_{l}
$$

and $\sigma_{1}, \ldots, \sigma_{m-1}$ with the property that

$$
\frac{\sigma_{i}}{R\left(x_{i}\right)} \leq C_{l}\left(\mathbf{B}^{\prime}\right) \leq C, \quad \frac{\sigma_{i}}{R\left(x_{i+1}\right)} \leq 1
$$

and

$$
B_{x_{i}}\left(R\left(x_{i}\right)\right) \subset B_{x_{i+1}}\left(\lambda^{2} \sigma_{i}\right)
$$


Since $x \in U(\mathbf{B})$, we have that $x_{m} \in U_{l}(\mathbf{B})=\Pi_{l}(U(\mathbf{B}))$. Thus, $R\left(x_{m}\right) \geq$ $R_{l}(\mathbf{B}) \geq \hat{R}_{l}$. Therefore, if we set $\sigma_{m}=2 \lambda^{-2}$, then

$$
\frac{\sigma_{m}}{R\left(x_{m+1}\right)} \leq 1
$$

since $R\left(x_{m+1}\right)=\infty$ and

$$
\frac{\sigma_{m}}{R\left(x_{m}\right)}=\frac{2 \lambda^{-2}}{R\left(x_{m}\right)} \leq \frac{2 \lambda^{-2}}{\hat{R}_{l}} \leq C .
$$

Furthermore, since the distance of $x$ to $S_{j}$ is equal to $1, R\left(x_{m}\right) \leq 1$. Hence

$$
B_{x_{m}}\left(R\left(x_{m}\right)\right) \subset B_{x_{m+1}}\left(\lambda^{2} \sigma_{m}\right) .
$$

This completes the inductive step and finishes the proof of Proposition 4.2.

Corollary 4.2. Let $\mathbf{B}$ be a dimension- $n$, codimension- $(n-k)$ local model, $g$ a Lipschitz Riemannian metric defined on $\mathbf{B}(r),(Y, d)$ an NPC space and $f:(\mathbf{B}(r), g) \rightarrow Y$ a finite energy map. Fix $\varrho \in(0,1)$. Then there exist $C \geq 1$ and $R_{0}>0$ depending on $g, \mathbf{B}(r)$ and $\varrho$ so that for every $x \in \mathbf{B}(\varrho r)$, there exist a sequence of points

$$
x=x_{1} \triangleright \cdots \triangleright x_{m}
$$

and a sequence of positive numbers

$$
\sigma_{1}, \ldots, \sigma_{m-1}
$$

so that for $i=1, \ldots, m-1$,

$$
\frac{\sigma_{i}}{r\left(x_{i}\right)} \leq C, \frac{\sigma_{i}}{r\left(x_{i+1}\right)} \leq 1, E_{x_{i}}\left(r\left(x_{i}\right)\right) \leq E_{x_{i+1}}\left(\sigma_{i}\right)
$$

and

$$
r\left(x_{m}\right) \geq R_{0}
$$

where $r(x)=\min \left\{\lambda \sigma_{0}, \lambda^{2} R(x)\right\}$ is as defined in (3.45).

Proof. We define $\mathcal{B}_{j}$ for $j=k, \ldots, n$ to be the set of model spaces so that $\mathbf{B}^{\prime} \in \mathcal{B}_{j}$ if and only if there exists $x \in S_{j}-S_{j-1}$ so that $B_{x}(R(x))$ is isometric to $\mathbf{B}^{\prime}(R(x))$. Then $\mathcal{B}_{k}, \ldots, \mathcal{B}_{n}$ satisfy conditions (1) and (2) of Proposition 4.2. 
Let $y \in \mathbf{B}(\varrho r)$ and assume $y \in S_{j}-S_{j-1}$. Choose $r_{y}>0$ sufficiently small so that $B_{y}\left(r_{y}\right)$ is compactly supported away from $S_{j-1}$ and $r_{y}<<\sigma_{0}$. In this way, we see that $\lambda^{2} R\left(y^{\prime}\right)=r\left(y^{\prime}\right)$ for $y^{\prime} \in B_{y}\left(r_{y}\right)$ and $R_{y}:=\inf \left\{r\left(y^{\prime}\right)\right.$ : $\left.y^{\prime} \in B_{y}\left(r_{y}\right) \cap S_{j}\right\}>0$. Choose a finite covering $\left\{B_{y_{l}}\left(r_{y_{l}}\right): l=1, \ldots, N\right\}$ of $\overline{\mathbf{B}\left(\frac{r}{2}\right)}$. Thus, given $x \in \mathbf{B}(\varrho r)$, there exists $l \in\{1, \ldots, N\}$ so that $x \in B_{y_{l}}\left(r_{y_{l}}\right)$. By construction, the point $y_{l}$ is an element of $S_{j}$ for some $j \in\{k, \ldots, n\}$ and $B_{y_{l}}\left(r_{y_{l}}\right)$ is isometric to $\mathbf{B}\left(r_{y_{l}}\right)$ for some $\mathbf{B} \in \mathcal{B}_{j}$. Applying Proposition 4.2 and noting that $r=\lambda^{2} R$, we obtain sequences $x_{1} \triangleright \cdots \triangleright x_{m}$ and $\sigma_{1}^{\prime}, \ldots, \sigma_{m-1}^{\prime}$ which satisfy

$$
\frac{\sigma_{i}^{\prime}}{\lambda^{-2} r\left(x_{i}\right)} \leq C, \frac{\sigma_{i}^{\prime}}{\lambda^{-2} r\left(x_{i+1}\right)} \leq 1 \text { and } B_{x_{i}}\left(\lambda^{-2} r\left(x_{i}\right)\right) \subset B_{x_{i+1}}\left(\lambda^{2} \sigma_{i}^{\prime}\right)
$$

by (4.3). If we set $\sigma_{i}=\lambda^{2} \sigma_{i}^{\prime}$, we obtain

$$
\frac{\sigma_{i}}{r\left(x_{i}\right)} \leq C, \frac{\sigma_{i}}{r\left(x_{i+1}\right)} \leq 1 \text { and } B_{x_{i}}\left(\lambda^{-2} r\left(x_{i}\right)\right) \subset B_{x_{i+1}}\left(\sigma_{i}\right) .
$$

Since $\lambda \in(0,1]$, the inclusion above shows that

$$
B_{x_{i}}\left(r\left(x_{i}\right)\right) \subset B_{x_{i+1}}\left(\sigma_{i}\right)
$$

which in turn implies

$$
E_{x_{i}}\left(r\left(x_{i}\right)\right) \leq E_{x_{i+1}}\left(\sigma_{i}\right) .
$$

Finally, if we set $R_{0}=\min \left\{R_{y_{1}}, . ., R_{y_{N}}\right\}$, then we obtain $r\left(x_{m}\right) \geq R_{0}>0$.

Proposition 4.3. Let $\mathbf{B}$ be a dimension-n, codimension- $(n-k)$ local model, $g$ a Lipschitz Riemannian metric defined on $\mathbf{B}(r),(Y, d)$ a metric space and $f:(\mathbf{B}(r), g) \rightarrow Y$ a finite energy map. Fix $\varrho \in(0,1)$ and suppose that for $x \in \mathbf{B}(\varrho r)$ there exist $\beta>0$ and $\hat{C} \geq 1$ so that

$$
\frac{E_{x}(\sigma)}{\sigma^{n-2+2 \beta}} \leq \hat{C} \frac{E_{x}(\rho)}{\rho^{n-2+2 \beta}}, \quad 0<\sigma \leq \rho \leq r(x),
$$

where $r(x)=\min \left\{\lambda \sigma_{0}, \lambda^{2} R(x)\right\}$ is as defined in (3.45). Then there exist $K$ and $R>0$ depending only on the total energy $E^{f}$ of $f$, the ellipticity constant $\lambda \in(0,1]$, the Lipschitz bound of $g, \mathbf{B}(r)$ and $\varrho$ so that

$$
E_{x}(\sigma) \leq K^{2} \sigma^{n-2+2 \beta}, \quad \forall x \in \mathbf{B}(\varrho r), \sigma<R .
$$


Proof. Set

$$
R:=\min \left\{R_{0}, \sigma_{0}\right\},
$$

where $R_{0}>0$ is as in Corollary 4.2 and $\sigma_{0} \in(0,1]$ as in Corollary 3.2,

$$
K_{0}:=\left(\hat{C} C^{n-2+2 \beta}\right)^{n} \frac{E^{f}}{R^{n-2+2 \beta}},
$$

where $C \geq 1$ as in Corollary 4.2 and

$$
K:=\left(\frac{2^{n} \kappa^{n}}{\lambda^{4} \sigma_{0}}\right)^{\frac{n}{2}-1+\beta} \max \left\{\sqrt{K_{0}}, \sqrt{E^{f}}\right\},
$$

where $\kappa \geq 1$ is defined in Corollary 4.1. Let $x \in \mathbf{B}(\varrho r)$ and $\sigma<R$.

Case 1. Assume that $\sigma \leq r(x)$. By (4.7),

$$
\frac{E_{x}(\sigma)}{\sigma^{n-2+2 \beta}} \leq \hat{C} \frac{E_{x}(r(x))}{r(x)^{n-2+2 \beta}} .
$$

Let $x=x_{1} \triangleright \cdots \triangleright x_{m}$ and $\sigma_{1}, \ldots, \sigma_{m-1}$ be as in Corollary 4.2. By (4.5) and $(4.7)$,

$$
\begin{aligned}
\frac{E_{x_{i}}\left(r\left(x_{i}\right)\right)}{r\left(x_{i}\right)^{n-2+2 \beta}} & \leq \frac{E_{x_{i+1}}\left(\sigma_{i}\right)}{r\left(x_{i}\right)^{n-2+2 \beta}} \\
& \leq C^{n-2+2 \beta} \frac{E_{x_{i+1}}\left(\sigma_{i}\right)}{\sigma_{i}^{n-2+2 \beta}} \\
& \leq \hat{C} C^{n-2+2 \beta} \frac{E_{x_{i+1}}\left(r\left(x_{i+1}\right)\right)}{r\left(x_{i+1}\right)^{n-2+2 \beta}}
\end{aligned}
$$

for $i=1, \ldots, m-1$. Additionally, by (4.6), we have that

$$
\frac{E_{x_{m}}\left(r\left(x_{m}\right)\right)}{r\left(x_{m}\right)^{n-2+2 \beta}} \leq \frac{E_{x_{m}}\left(r\left(x_{m}\right)\right)}{R^{n-2+2 \beta}} \leq \frac{E^{f}}{R^{n-2+2 \beta}}
$$

and hence

$$
\frac{E_{x_{1}}(\sigma)}{\sigma^{n-2+2 \beta}} \leq\left(\hat{C} C^{n-2+2 \beta}\right)^{m} \frac{E^{f}}{R^{n-2+2 \beta}} .
$$

This implies

$$
E_{x}(\sigma) \leq K^{2} \sigma^{n-2+2 \beta} \text { whenever } \sigma \leq r(x)
$$


Case 2. Alternately, assume $r(x)<\sigma$. Since $r(x)=\min \left\{\lambda \sigma_{0}, \lambda^{2} R(x)\right\}$, either

$$
\lambda \sigma_{0}<\sigma \quad \text { or } \quad R(x)<\frac{\sigma}{\lambda^{2}} .
$$

First consider the case when $\lambda \sigma_{0}<\sigma$. Then

$$
E_{x}(\sigma) \leq E_{x}(\sigma)\left(\frac{\sigma}{\lambda \sigma_{0}}\right)^{n-2+2 \beta} \leq \frac{E^{f}}{\left(\lambda \sigma_{0}\right)^{n-2+2 \beta}} \sigma^{n-2+2 \beta} \leq K^{2} \sigma^{n-2+2 \beta} .
$$

Next assume $R(x)<\frac{\sigma}{\lambda^{2}}$. The fact that $R\left(x^{\prime}\right)=\infty$ for every $x^{\prime} \in D=S_{k}$ implies that $x \notin D=S_{k}$. So let $i \in\{k+1, \ldots, n\}$ be so that $x \in S_{i}-S_{i-1}$. Furthermore, let $x=y_{i}, \ldots, y_{k} \in D=S_{k}$ where $y_{j-1} \in \Pi_{j-1}\left(y_{j}\right)$. We now follow the following finite step procedure.

Step 1. Since $R\left(y_{i}\right)<\frac{\sigma}{\lambda^{2}}$, there exists $l_{1} \in\{k+1, \ldots, i-1\}$ so that

$$
R\left(y_{m}\right)<\frac{2^{i-m} \sigma}{\lambda^{2}}, \forall m=l_{1}+1, \ldots, i .
$$

Thus, we can apply Corollary 4.1 to obtain

$$
\left|y_{m}-y_{m-1}\right|<\kappa R\left(y_{m}\right)<\frac{2^{i-m} \kappa}{\lambda^{2}} \sigma .
$$

This implies

$$
B_{y_{i}}\left(\frac{\kappa}{\lambda^{2}} \sigma\right) \subset B_{y_{i-1}}\left(\frac{2 \kappa}{\lambda^{2}} \sigma\right) \subset \cdots \subset B_{y_{l_{1}}}\left(\frac{2^{i-l_{1}} \kappa}{\lambda^{2}} \sigma\right) .
$$

Since $\frac{\kappa}{\lambda^{2}} \geq 1$, we also have

$$
B_{y_{i}}(\sigma) \subset B_{y_{i}}\left(\frac{\kappa}{\lambda^{2}} \sigma\right)
$$

The above inclusions imply

$$
E_{x}(\sigma) \leq E_{y_{l_{1}}}\left(\frac{2^{i-l_{1}} \kappa}{\lambda^{2}} \sigma\right)
$$

Now we consider the two possibilities, either

$$
\frac{2^{i-l_{1}} \kappa}{\lambda^{2}} \sigma \leq r\left(y_{l_{1}}\right) \quad \text { or } \quad r\left(y_{l_{1}}\right)<\frac{2^{i-l_{1}} \kappa}{\lambda^{2}} \sigma .
$$


In the former possibility, we use (4.8) and Case 1 to see that

$$
E_{x}(\sigma) \leq E_{y_{l_{1}}}\left(\frac{2^{i-l_{1}} \kappa}{\lambda^{2}} \sigma\right) \leq K_{0}^{2}\left(\frac{2^{i-l_{1}} \kappa}{\lambda^{2}} \sigma\right)^{n-2+2 \beta} \leq K^{2} \sigma^{n-2+2 \beta}
$$

In the latter possibility, either

$$
\text { (A) } \sigma_{0}<\frac{2^{i-l_{1}} \kappa}{\lambda^{4}} \sigma \quad \text { or } \quad(\mathrm{B}) \quad R\left(y_{l_{1}}\right)<\frac{2^{i-l_{1}} \kappa}{\lambda^{4}} \sigma \text {. }
$$

In Case (A), we use (4.8) and the fact that $1 \leq \frac{2^{i-l_{1}} \kappa}{\lambda^{4} \sigma_{0}} \sigma$ to obtain

$$
E_{x}(\sigma) \leq E_{y_{l_{1}}}\left(\frac{2^{i-l_{1}} \kappa}{\lambda^{2}} \sigma\right)\left(\frac{2^{i-l_{1}} \kappa}{\lambda^{4} \sigma_{0}}\right)^{n-2+2 \beta} \sigma^{n-2+2 \beta} \leq K^{2} \sigma^{n-2+2 \beta} .
$$

If $(\mathrm{B})$ is true, then we proceed to Step 2.

Step 2. Since we are assuming $R\left(y_{l_{1}}\right)<\frac{2^{i-l_{1}} \kappa}{\lambda^{4}} \sigma$, there exists $l_{2} \in\{k+1, \ldots$, $\left.l_{1}-1\right\}$ so that

$$
R\left(y_{m}\right)<\frac{2^{i-m} \kappa}{\lambda^{4}} \sigma, \forall m=l_{2}+1, \ldots, l_{1} .
$$

Thus, we can apply Corollary 4.1 to obtain

$$
\left|y_{m}-y_{m-1}\right|<\kappa R\left(y_{m}\right)<\frac{2^{i-m} \kappa^{2}}{\lambda^{4}} \sigma, \forall m=l_{2}+1, \ldots, l_{1} .
$$

Hence

$$
B_{y_{l_{1}}}\left(\frac{2^{i-l_{1}} \kappa^{2}}{\lambda^{4}} \sigma\right) \subset \cdots \subset B_{y_{l_{2}}}\left(\frac{2^{i-l_{2}} \kappa^{2}}{\lambda^{4}} \sigma\right) .
$$

Combined with (4.8), this implies

$$
E_{x}(\sigma) \leq E_{y_{l_{2}}}\left(\frac{2^{i-l_{2}} \kappa^{2}}{\lambda^{4}} \sigma\right)
$$

We now continue. In the similar way as in Step 1 , we prove $E_{x}(\sigma) \leq K^{2}$ $\sigma^{n-2+2 \beta}$ or we continue to Step 3 where we assume $R\left(y_{l_{2}}\right)<\frac{2^{i-l_{2}} \kappa^{2}}{\lambda^{4}} \sigma$. At Step $S$ of this procedure, we produce an integer $l_{S} \in\{k, \ldots, i\}$ and this procedure terminates after a finite number of steps since $l_{S}>l_{S+1}$. Finally, observe that in order to prove our assertion, we must show that if $N$ is the number of steps taken and if $y_{l_{N}}=y_{k}$ then the case corresponding to (B) (i.e., the case that $R\left(y_{l_{N}}\right)<\frac{2^{i-l_{N} \kappa^{N}}}{\lambda^{4}} \sigma$ ) does not occur. This is true because $R\left(y_{l_{N}}\right)=R\left(y_{k}\right)=\infty$ and this completes the proof. 
The following is a version of the Campanato Lemma (cf.[16, Lemma 1]).

Lemma 4.1. Let $\mathbf{B}$ be a dimension- $n$, codimension- $(n-k)$ local model, $g$ a Lipschitz Riemannian metric defined on $\mathbf{B}(r),(Y, d)$ an NPC space and $f:(\mathbf{B}(r), g) \rightarrow Y$ an $L^{2}$-map. Fix $\varrho \in(0,1)$. If there exist $K>0, R \in$ $(0,(1-\varrho) r)$ and $\beta \in(0,1]$ such that

$$
\inf _{Q \in Y} \sigma^{-n} \int_{B_{x}(\sigma)} d^{2}(f, Q) d \mu_{g} \leq K^{2} \sigma^{2 \beta}, \forall x \in \mathbf{B}(\varrho r) \text { and } \sigma \in(0, R),
$$

then (there exists a representative in the $L^{2}$-equivalence class of $f$ which we still denote by $f$ ) such that

$$
d(f(x), f(y)) \leq C|x-y|^{\beta}, \forall x, y \in \mathbf{B}(\varrho r)
$$

with $C$ depending on $K, r, R, \beta$, $\varrho$ and $\mathbf{B}(r)$.

Proof. We will use $C$ to denote a generic constant that depends only on $K$, $r, R, \beta, \varrho$ and $\mathbf{B}$. For $x \in \mathbf{B}(\varrho r)$ and $\sigma \in(0, R)$, let $Q_{x, \sigma} \in Y$ be such that

$$
\frac{1}{\operatorname{Vol}\left(B_{x}(\sigma)\right)} \int_{B_{x}(\sigma)} d^{2}\left(f, Q_{x, \sigma}\right) d \mu_{g}=\inf _{Q \in Y} \frac{1}{\operatorname{Vol}\left(B_{x}(\sigma)\right)} \int_{B_{x}(\sigma)} d^{2}(f, Q) d \mu_{g} .
$$

For the existence of $Q_{x, \sigma}$, see Lemma 2.5.1 of [11]. Furthermore,

$$
\begin{aligned}
\left(\frac{\sigma}{2}\right)^{-n} \int_{B_{x}(\sigma / 2)} d^{2}\left(f, Q_{x, \sigma / 2}\right) d \mu_{g} & \leq\left(\frac{\sigma}{2}\right)^{-n} \int_{B_{x}(\sigma / 2)} d^{2}\left(f, Q_{x, \sigma}\right) d \mu_{g} \\
& \leq\left(\frac{\sigma}{2}\right)^{-n} \int_{B_{x}(\sigma)} d^{2}\left(f, Q_{x, \sigma}\right) d \mu_{g} \\
& \leq 2^{n} C \sigma^{2 \beta}
\end{aligned}
$$

Thus,

$$
\begin{aligned}
& \left(\frac{\sigma}{2}\right)^{-n} \int_{B_{x}(\sigma / 2)} d^{2}\left(Q_{x, \sigma}, Q_{x, \sigma / 2}\right) d \mu_{g} \\
& \quad \leq\left(\frac{\sigma}{2}\right)^{-n} \int_{B_{x}(\sigma / 2)} 2 d^{2}\left(f, Q_{x, \sigma}\right)+2 d^{2}\left(f, Q_{x, \sigma / 2}\right) d \mu_{g} \\
& \leq 2^{n+2} C \sigma^{2 \beta}
\end{aligned}
$$

which implies

$$
d\left(Q_{x, \sigma}, Q_{x, \sigma / 2}\right) \leq C \sigma^{\beta} .
$$


Apply the above inequality with $\sigma=R / 2^{k}$ and sum to obtain

$$
\begin{aligned}
d\left(Q_{x, R / 2^{\nu}}, Q_{x, R / 2^{\nu^{\prime}}}\right) & \leq \sum_{k=\nu}^{\nu^{\prime}-1} d\left(Q_{x, R / 2^{k}}, Q_{x, R / 2^{k+1}}\right) \\
& \leq C \sum_{k=\nu}^{\nu^{\prime}-1}\left(\frac{1}{2^{\beta}}\right)^{\nu} .
\end{aligned}
$$

The sum on the right-hand side is a partial sum of a geometric series and hence $\left\{Q_{x, R / 2^{\nu}}\right\}_{\nu=0,1, \ldots}$ is a Cauchy sequence. Since $Y$ is complete, there exists $Q_{x} \in Y$ such that

$$
\lim _{\nu \rightarrow \infty} d\left(Q_{x}, Q_{x, R / 2^{\nu}}\right)=0
$$

Again using (4.10), we obtain

$$
\begin{aligned}
d\left(Q_{x, R / 2^{\nu}}, Q_{x}\right) & =\lim _{\nu^{\prime} \rightarrow \infty} d\left(Q_{x, R / 2^{\nu}}, Q_{x, R / 2^{\nu^{\prime}}}\right) \\
& =\lim _{\nu^{\prime} \rightarrow \infty} \sum_{k=\nu}^{\nu^{\prime}-1} d\left(Q_{x, R / 2^{k}}, Q_{x, R / 2^{k+1}}\right) \\
& \leq C\left(\frac{1}{2^{\nu}}\right)^{\beta} .
\end{aligned}
$$

Thus, using $d^{2}\left(f, Q_{x}\right) \leq 2 d^{2}\left(f, Q_{x, \sigma}\right)+2 d^{2}\left(Q_{x, \sigma}, Q_{x}\right)$, we obtain

$$
\sigma^{-n} \int_{B_{x}(\sigma)} d^{2}\left(f, Q_{x}\right) d \mu_{g} \leq C \sigma^{2 \beta}
$$

for every $\sigma=\frac{R}{2^{\nu}}, \nu=0,1, \ldots$ On the other hand, for an $\sigma \in(0, R]$, there is an integer $\nu \geq 0$ such that $R / 2^{\nu+1}<\sigma \leq R / 2^{\nu}$ and we can conclude that 
(4.11) holds for all $\sigma \in(0, R]$. Let $x, y \in \mathbf{B}(\varrho r)$ with $|x-y|<R$ and let $x_{\frac{1}{2}}$ be a point in $\mathbf{B}(r)$ such that $2\left|x-x_{\frac{1}{2}}\right|=2\left|y-x_{\frac{1}{2}}\right|=|x-y|=: \sigma$. Using the fact that $B_{x_{\frac{1}{2}}}\left(\frac{\sigma}{2}\right) \subset B_{x}(\sigma) \cap B_{y}(\sigma)$, we obtain

$$
\begin{aligned}
& d^{2}\left(Q_{x}, Q_{y}\right) \\
& \quad \leq \frac{1}{\operatorname{Vol}\left(B_{x_{\frac{1}{2}}}\left(\frac{\sigma}{2}\right)\right)} \int_{B_{x_{\frac{1}{2}}}\left(\frac{\sigma}{2}\right)} d^{2}\left(Q_{x}, Q_{y}\right) d \mu_{g} \\
& \quad \leq C \sigma^{-n} \int_{B_{x_{\frac{1}{2}}}\left(\frac{\sigma}{2}\right)} 2 d^{2}\left(f, Q_{x}\right)+2 d^{2}\left(f, Q_{y}\right) d \mu_{g} \\
& \quad \leq C\left(\sigma^{-n} \int_{B_{x}(\sigma)} d^{2}\left(f, Q_{x}\right) d \mu_{g}+\sigma^{-n} \int_{B_{y}(\sigma)} d^{2}\left(f, Q_{y}\right) d \mu_{g}\right) \\
& \quad \leq C \sigma^{2 \beta}=C|x-y|^{2 \beta} .
\end{aligned}
$$

For a pair of points $x, y \in \mathbf{B}(\varrho r)$, we can choose a sequence $z_{0}=x, \ldots, z_{k}=y$ such that $\left|z_{i}-z_{i+1}\right| \leq R$ and $k \leq 2 \varrho r / R$. Applying the above inequality to pairs $z_{i}, z_{i+1}$ and summing, we obtain

$$
d^{2}\left(Q_{x}, Q_{y}\right) \leq \frac{2 \varrho r}{R} C|x-y|^{2 \beta}, \forall x, y \in \mathbf{B}(\varrho r)
$$

Finally, we will show that $f(x)=Q_{x}$ for a.e. $x \in \mathbf{B}(\varrho r)$. This of course will complete the proof of the lemma. It suffices to show that $f(x)=Q_{x}$ for a.e. $x$ contained in an interior of a wedge. In fact, by using compact exhaustion, it suffices to show $f(x)=Q_{x}$ for $x \in \Omega$ where $\Omega$ is an Euclidean domain contained in the interior of every wedge of $\mathbf{B}(\varrho r)$. We first prove:

\section{Claim. If}

$$
E_{\epsilon}=\left\{x \in \Omega: \limsup _{\sigma \rightarrow 0} \frac{1}{\operatorname{Vol}\left(B_{x}(\sigma)\right)} \int_{B_{x}(\sigma)} d^{2}(f, f(x)) d \mu_{g}>4 \epsilon\right\}
$$

then

$$
\mu_{g}\left(E_{\epsilon}\right)=0 .
$$


To prove this claim, we let $g: \Omega \rightarrow Y$ be a Lipschitz map which approximates $f$ in $L^{2}$ (cf. Section 1.5 of [12]). Then

$$
\begin{aligned}
& \frac{1}{\operatorname{Vol}\left(B_{x}(\sigma)\right)} \int_{B_{x}(\sigma)} d^{2}(f, f(x)) d \mu_{g} \\
& \quad \leq \frac{2}{\operatorname{Vol}\left(B_{x}(\sigma)\right)} \int_{B_{x}(\sigma)} d^{2}(f, g) d \mu_{g}+\frac{2}{\operatorname{Vol}\left(B_{x}(\sigma)\right)} \int_{B_{x}(\sigma)} d^{2}(g, g(x)) d \mu_{g} \\
& \quad+\frac{2}{\operatorname{Vol}\left(B_{x}(\sigma)\right)} \int_{B_{x}(\sigma)} d^{2}(g(x), f(x)) d \mu_{g} \\
& \quad \leq \frac{2}{\operatorname{Vol}\left(B_{x}(\sigma)\right)} \int_{B_{x}(\sigma)} d^{2}(f, g) d \mu_{g}+\frac{2}{\operatorname{Vol}\left(B_{x}(\sigma)\right)} \int_{B_{x}(\sigma)} d^{2}(g, g(x)) d \mu_{g} \\
& \quad+2 d^{2}(g(x), f(x)) .
\end{aligned}
$$

Since $g$ is continuous, the second term on the right-hand side approaches 0 as $\sigma \rightarrow 0$. Thus,

$$
\limsup _{\sigma \rightarrow 0} \frac{1}{\operatorname{Vol}\left(B_{x}(\sigma)\right)} \int_{B_{x}(\sigma)} d^{2}(f, f(x)) d \mu_{g} \leq 2 \mathrm{H} L(x)+2 d^{2}(g(x), f(x)),
$$

where

$$
H L(x):=\sup _{r>0} \frac{1}{\operatorname{Vol}\left(B_{x}(r)\right)} \int_{B_{x}(r)} d^{2}(f, g) d \mu_{g}
$$

If

$$
\limsup _{\sigma \rightarrow 0} \frac{1}{\operatorname{Vol}\left(B_{x}(\sigma)\right)} \int_{B_{x}(\sigma)} d^{2}(f, f(x)) d \mu_{g}>4 \epsilon
$$

then either

$$
H L(x)>\epsilon \quad \text { or } \quad d^{2}(g(x), f(x))>\epsilon .
$$

By the Hardy-Littlewood maximal theorem there exists a constant $c>0$ such that

$$
\mu_{g}(\{x \in \Omega: \mathrm{H} L(x)>\epsilon\}) \leq \frac{c}{\epsilon} \int_{\Omega} d^{2}(f, g) d \mu_{g}
$$

and the Markov inequality says

$$
\mu_{g}\left(\left\{x \in \Omega: d^{2}(f, g)>\epsilon\right\}\right) \leq \frac{1}{\epsilon} \int_{\Omega} d^{2}(f, g) d \mu_{g}
$$

Hence

$$
\mu_{g}\left(E_{\epsilon}\right)<\frac{c+1}{\epsilon} \int_{\Omega} d^{2}(f, g) d \mu_{g}
$$

Since $g$ can be chosen such that the integral on the right-hand side is arbitrarily small, we have proved the claim. 
It follows that

$$
\lim _{\sigma \rightarrow 0} \frac{1}{\operatorname{Vol}\left(B_{x}(\sigma)\right)} \int_{B_{x}(\sigma)} d^{2}(f, f(x)) d \mu_{g}=0 \text { for a.e. } x \in \Omega .
$$

Furthermore,

$$
\begin{aligned}
& d\left(f(x), Q_{x}\right) \\
& =\frac{1}{\operatorname{Vol}\left(B_{x}(\sigma)\right)} \int_{B_{x}(\sigma)} d\left(f(x), Q_{x}\right) d \mu_{g} \\
& \leq \leq \frac{2}{\operatorname{Vol}\left(B_{x}(\sigma)\right)} \int_{B_{x}(\sigma)} d^{2}(f, f(x)) d \mu_{g}+\frac{2}{\operatorname{Vol}\left(B_{x}(\sigma)\right)} \int_{B_{x}(\sigma)} d^{2}\left(f, Q_{x}\right) d \mu_{g} \\
& \leq \leq \frac{2}{\operatorname{Vol}\left(B_{x}(\sigma)\right)} \int_{B_{x}(\sigma)} d^{2}(f, f(x)) d \mu_{g}+\frac{4}{\operatorname{Vol}\left(B_{x}(\sigma)\right)} \int_{B_{x}(\sigma)} d^{2}\left(f, Q_{x, \sigma}\right) d \mu_{g} \\
& \quad+\frac{4}{\operatorname{Vol}\left(B_{x}(\sigma)\right)} \int_{B_{x}(\sigma)} d^{2}\left(Q_{x}, Q_{x, \sigma}\right) d \mu_{g} \\
& \leq \frac{6}{\operatorname{Vol}\left(B_{x}(\sigma)\right)} \int_{B_{x}(\sigma)} d^{2}(f, f(x)) d \mu_{g}+4 d^{2}\left(Q_{x}, Q_{x, \sigma}\right) .
\end{aligned}
$$

Hence,

$$
\begin{aligned}
& d\left(f(x), Q_{x}\right) \\
& \leq 6 \lim _{\nu \rightarrow \infty} \frac{1}{\operatorname{Vol}\left(B_{x}\left(R / 2^{\nu}\right)\right)} \int_{B_{x}\left(R / 2^{\nu}\right)} d^{2}(f, f(x)) d \mu_{g} \\
& \quad+4 \lim _{\nu \rightarrow \infty} d^{2}\left(Q_{x}, Q_{x, R / 2^{\nu}}\right) \\
& \quad=0 \quad \text { for a.e. } x \in \Omega .
\end{aligned}
$$

This completes the proof.

By combining the previous lemma with the Poincare Lemma 2.1, we obtain

Proposition 4.4. Let $\mathbf{B}$ be a dimension- $n$, codimension- $(n-k)$ local model, $g$ a Lipschitz Riemannian metric defined on $\mathbf{B}(r),(Y, d)$ an NPC space and $f:(\mathbf{B}(r), g) \rightarrow Y$ a finite energy map. Fix $\varrho \in(0,1)$. Suppose that there exists $R>0$ so that for every $x \in \mathbf{B}(\varrho r), \sigma<R$,

$$
E_{x}(\sigma) \leq K^{2} \sigma^{n-2+2 \beta}
$$


Then

$$
d(f(x), f(y)) \leq C|x-y|^{\beta}, \forall x, y \in \mathbf{B}(\varrho r)
$$

with $C$ depending on $K, R, \varrho, \mathbf{B}(r)$ and the ellipticity constant of $g$.

Theorem 4.1. Let $\mathbf{B}$ be a local model, $g$ a Lipschitz Riemannian metric defined on $\mathbf{B}(r),(Y, d)$ an NPC space, $f:(\mathbf{B}(r), g) \rightarrow Y$ a harmonic map and $\alpha_{x}$ the order of $f$ at $x$. If $\alpha_{x} \geq \alpha>0$ for all $x \in \mathbf{B}(\varrho r)$ where $\varrho \in(0,1)$, then there exists $C$ depending only on the Lipchitz bound and ellipticity constant of $g, E^{f}, \mathbf{B}(r)$ and $\varrho$ such that

$$
d(f(x), f(y)) \leq C|x-y|^{\alpha}, \quad \forall x, y \in \mathbf{B}(\varrho r) .
$$

Proof. The result follows immediately from inequality (3.43) of Propositions $3.1,4.3$ and 4.4 .

Theorem 4.2. Let $\mathbf{B}$ be a local model, $g$ a Lipschitz Riemannian metric defined on $\mathbf{B}(r),(Y, d)$ an NPC space and $f:(\mathbf{B}(r), g) \rightarrow Y$ a harmonic map. For $\varrho \in(0,1)$, there exist $C$ and $\gamma>0$ depending only on the Lipchitz bound and ellipticity constant of $g, E^{f}, \mathbf{B}(r)$ and $\varrho$ such that

$$
d(f(x), f(y)) \leq C|x-y|^{\gamma}, \quad \forall x, y \in \mathbf{B}(\varrho r) .
$$

Proof. The result follows immediately from inequality (3.44) of Propositions $3.1,4.3$ and 4.4 .

By using Proposition 2.1 we obtain the following:

Theorem 4.3. Let $B(r)$ be a ball or radius $r$ around a point in an admissible complex $X$ endowed with a Lipschitz Riemannian metric $g$ and $(Y, d)$ an NPC space. If $f:(B(r), g) \rightarrow Y$ is a harmonic map and $\varrho \in(0,1)$, then there exist $C$ and $\gamma>0$ so that

$$
d(f(x), f(y)) \leq C|x-y|^{\gamma}, \quad \forall x, y \in B(\varrho r) .
$$

Here, $C$ and $\gamma$ only depend the total energy $E^{f}$ of the map $f,(B(r), g)$ and $\varrho$.

\section{Convergence in the pull back sense}

Given a metric space $(Y, d)$ and a map $u: \mathbf{B}(r) \rightarrow(Y, d)$, we recall the following construction of [12]. First, we let $\Omega_{0}=\mathbf{B}(r), u_{0}=u$ and $d_{0}: \Omega_{0} \times$ $\Omega_{0} \rightarrow \mathbf{R}^{+} \cup\{0\}$ be the pseudodistance function $d_{0}(x, y)=d\left(u_{0}(x), u_{0}(y)\right)$. 
Next, we inductively define $\Omega_{i+1}=\Omega_{i} \times \Omega_{i} \times[0,1]$ and identify $\Omega_{i}$ as a subset of $\Omega_{i+1}$ by the inclusion map $x \mapsto(x, x, 0)$. Extend $u_{i}: \Omega \rightarrow(Y, d)$ to $u_{i+1}: \Omega \rightarrow(Y, d)$ by

$$
u_{i+1}(x, y, \lambda)=(1-\lambda) u_{i}(x)+\lambda u_{i}(y)
$$

and let

$$
d_{i+1}(x, y)=d\left(u_{i+1}(x), u_{i+1}(y)\right) .
$$

Thus,

$$
\begin{gathered}
d_{i+1}((x, x, 0),(y, y, 0))=d_{i}(x, y), \\
d_{i+1}((x, y, \lambda),(x, y, \mu))=|\lambda-\mu| d_{i}(x, y)
\end{gathered}
$$

and

$$
\begin{aligned}
& d_{i+1}(z,(x, y, \lambda)) \leq(1-\lambda) d_{i+1}(z,(x, x, 0)) \\
& \quad+\lambda d_{i+1}(z,(y, y, 0))-\lambda(1-\lambda) d_{i+1}((x, x, 0),(y, y, 0)) .
\end{aligned}
$$

Let $\Omega_{\infty}=\cup \Omega_{i}$ and define $u_{\infty}: \Omega_{\infty} \rightarrow(Y, d)$ by setting $u_{\infty}=u_{i}$ on $\Omega_{i}$. With $d_{\infty}(x, y):=d\left(u_{\infty}(x), u_{\infty}(y)\right)$, define $\left(Y_{\infty}, d_{\infty}\right)$ as the completion of the quotient space from $\left(\Omega_{\infty}, d_{\infty}\right)$ and let $\pi: \Omega_{\infty} \rightarrow Y_{\infty}$ be the natural projection map. Equation (5.1) implies that the metric space $\left(Y_{\infty}, d_{\infty}\right)$ is an NPC space. The unique extension of $u_{\infty}$ to $Y_{\infty}$ is an isometry $U:\left(Y_{\infty}, d_{\infty}\right) \rightarrow$ $\mathcal{C}(u(\mathbf{B}(r))) \subset Y$ to the closed convex hull of the image of $u$. Furthermore, if $\iota: \mathbf{B}(r)=\Omega_{0} \rightarrow \Omega_{\infty}$ is the inclusion map, then $u=U \circ \pi \circ \iota$, (cf.[12]).

Definition 5.1. Let $v_{k}: \mathbf{B}(r) \rightarrow\left(Y_{k}, d_{k}\right)$ be a sequence of maps to NPC spaces. We say $v_{k}$ converges to $v_{*}$ in the pullback sense if there exists a pseudodistance function $d_{*}: \Omega_{\infty} \times \Omega_{\infty} \rightarrow \mathbf{R}^{+} \cup\{0\}$ with the following property. Let $\left(Y_{*}, d_{*}\right)$ be the completed quotient space from $\left(\Omega_{\infty}, d_{*}\right)$ and $\pi: \Omega_{\infty} \rightarrow Y_{*}$ the natural projection map. Furthermore, let $v_{k}=u$ in the above paragraph and let $d_{k, \infty}: \Omega_{\infty} \times \Omega_{\infty} \rightarrow \mathbf{R}^{+} \cup\{0\}$ the corresponding pullback distance function of $v_{k, \infty}$ replacing $u_{\infty}$ above. Then $d_{k, \infty}$ converges pointwise to $d_{*}$ and $v_{*}=\pi \circ \iota$.

Remark 5.1. If we let $v_{*}=u$ with $u$ as in the paragraph preceeding the definition above and $d_{*, i}\left(d_{*, \infty}\right.$ resp.) the corresponding pullback distance function of $v_{*, i}=u_{i}\left(v_{*, \infty}=u_{\infty}\right.$ resp.), then $d_{*}=d_{*, \infty}$. 
Definition 5.2. Suppose $v_{k}$ converge to $v_{*}$ in the pullback sense. Let $d_{k, i}$ (resp. $d_{*, i}$ ) be the corresponding pullback distance function to $v_{k, i}: \Omega_{i} \rightarrow$ $\left(Y_{k}, d_{k}\right)$ (resp. $v_{*, i}: \Omega_{i} \rightarrow\left(Y_{*}, d_{*}\right)$ ). We say that the convergence is locally uniform if the convergence of $d_{k, i}$ to the limit $d_{*, i}$ is uniform on each compact subset of $\Omega_{i} \times \Omega_{i}$. In this case, we also say that $v_{*}$ is a locally uniform limit of $v_{k}$.

Proposition 5.1. Let $v_{k}: \mathbf{B}(r) \rightarrow\left(Y_{k}, d_{k}\right)$ be a sequence of maps to $N P C$ spaces for which there is uniform modulus of continuity control, i.e., assume for each $x \in \mathbf{B}(r)$ and $R>0$ there is a positive function $\omega(x, R)$ which is monotone in $R$, satisfying

$$
\lim _{R \rightarrow 0} \omega(x, R)=0,
$$

and so that for each $k \in \mathbf{Z}$

$$
\max _{y \in B(x, R)} d\left(v_{k}(x), v_{k}(y)\right) \leq \omega(x, R) .
$$

Then there is an NPC space $\left(Y_{*}, d_{*}\right)$ and a subsequence $v_{k^{\prime}}$ of the $v_{k}$ which converges locally uniformly in the pullback sense to a limit map $v_{*}: \mathbf{B}(r) \rightarrow$ $\left(Y_{*}, d_{*}\right)$, and $v_{*}$ satisfies the same modulus of continuity estimates. Here, $\left(Y_{*}, d_{*}\right)$ is the completed quotient of $\left(\Omega_{\infty}, d_{*, \infty}\right)$ where $d_{*, \infty}=\lim _{k^{\prime} \rightarrow \infty} d_{k^{\prime}, \infty}$.

Proof. The proposition follows from the argument of the proof of Lemma 3.1 and Proposition 3.7 in [12] since the fact that $\mathbf{B}(r)$ is not a Riemannian domain plays no consequence in the argument.

\section{The tangent map}

Let $\mathbf{B}$ be a dimension- $n$, codimension- $\nu$ local model and $g$ a normalized (i.e., $\left.g_{i j}(0)=\delta_{i j}\right)$ Lipschitz metric on $\mathbf{B}(1)$. Given $r \in(0,1), f: \mathbf{B}(r) \rightarrow(Y, d)$ and $\lambda>0$, define the $\lambda$-blow up map $f_{\lambda}: \mathbf{B}\left(\frac{r}{\lambda}\right) \rightarrow\left(Y, d_{\lambda}\right)$ by setting

$$
\begin{aligned}
g_{\lambda}(x) & =g(\lambda x), \\
\mu_{\lambda} & =\left(\lambda^{1-n} I(\lambda)\right)^{1 / 2}, \\
d_{\lambda}(p, q) & =\mu_{\lambda}^{-1} d(p, q), \\
f_{\lambda}(x) & =f(\lambda x),
\end{aligned}
$$

Definition 6.1. If there exist $\lambda_{k} \rightarrow 0$ and an NPC space $\left(Y_{*}, d_{*}\right)$ so that $f_{\lambda_{k}}$ converges locally uniformly in the pullback sense to $f_{*}: \mathbf{B} \rightarrow\left(Y_{*}, d_{*}\right)$, then $f_{*}$ is called a tangent map of $f$. 
Lemma 6.1. A harmonic map $f:(\mathbf{B}, g) \rightarrow(Y, d)$ has a non-constant tangent map $f_{*}$ which satisfies

$$
d\left(f_{*}(x), f_{*}(y)\right) \leq C^{\prime}|x-y|^{\gamma},
$$

where $C^{\prime}$ and $\gamma$ are only depending on the Lipschitz bound of $g$ and $E^{f}$.

Proof. By change of variables

$$
\int_{\mathbf{B}(\sigma)}\left|\nabla f_{\lambda}\right|_{g_{\lambda}}^{2} d \mu_{g_{\lambda}}=\mu_{\lambda}^{-2} \lambda^{2-n} \int_{\mathbf{B}(\lambda \sigma)}|\nabla f|_{g}^{2} d \mu_{g}
$$

and

$$
\int_{\partial \mathbf{B}(\sigma)} d_{\lambda}^{2}\left(f_{\lambda}, f_{\lambda}(0)\right) d \Sigma_{g_{\lambda}}=\mu_{\lambda}^{-2} \lambda^{1-n} \int_{\partial \mathbf{B}(\lambda \sigma)} d^{2}(f, f(0)) d \Sigma_{g}
$$

Thus, the definition of $\mu_{\lambda}$ implies

$$
\int_{\partial \mathbf{B}(1)} d_{\lambda}^{2}\left(f_{\lambda}, f_{\lambda}(0)\right) d \Sigma_{g_{\lambda}}=1
$$

and Corollary 3.2 implies

$$
\lim _{\lambda \rightarrow 0} \frac{\int_{\mathbf{B}(1)}\left|\nabla f_{\lambda}\right|_{g_{\lambda}}^{2} d \mu_{g_{\lambda}}}{\int_{\partial \mathbf{B}(1)} d_{\lambda}^{2}\left(f_{\lambda}, f_{\lambda}(0)\right) d \Sigma_{g_{\lambda}}}=\lim _{\lambda \rightarrow 0} \frac{\lambda E(\lambda)}{I(\lambda)}=\alpha
$$

Consequently, by choosing $\lambda$ sufficiently small, we have

$$
\int_{\mathbf{B}(1)}\left|\nabla f_{\lambda}\right|^{2} d \mu_{g_{\lambda}} \leq 2 \alpha
$$

Since $g_{i j}$ is Lipschitz, we have

$$
\left|g_{i j}(x)-\delta_{i j}\right| \leq c|x| \text { and }\left|g^{i j}(x)-\delta_{i j}\right| \leq c|x| .
$$

Hence,

$$
\left|\left(g_{\lambda}\right)_{i j}(x)-\delta_{i j}\right| \leq c \lambda|x| \text { and }\left|\left(g_{\lambda}\right)^{i j}-\delta_{i j}\right| \leq c \lambda|x| .
$$

Therefore, there exists a uniform Lipschitz bound of the family of metrics $\left\{g_{\lambda}\right\}$ independent of $\lambda$. This implies

$$
d_{\lambda}\left(f_{\lambda}(x), f_{\lambda}(y)\right) \leq C|x-y|^{\gamma} \forall x, y \in \mathbf{B}(r),
$$


where $C$ and $\gamma$ are independent of $\lambda$ by Theorem 4.2. Thus, we have uniform modulus of continuity control for the sequence $f_{\lambda}$, then by Proposition 5.1, there exists a sequence $\lambda_{k} \rightarrow 0$ and an NPC space $\left(Y_{*}, d_{*}\right)$ so that $f_{\lambda_{k}}$ converges locally uniformly in the pullback sense to a limit map $f_{*}: \mathbf{B}(1) \rightarrow\left(Y_{*}, d_{*}\right)$. The fact that $f_{*}$ is non-constant follows immediately from the proof of Proposition 3.3 of [9].

Lemma 6.2. Let $g$ be a normalized metric on $\mathbf{B}(r), f:(\mathbf{B}(r), g) \rightarrow(Y, d)$ a harmonic map and $f_{\lambda}: \mathbf{B}\left(\frac{r}{\lambda}\right) \rightarrow\left(Y, d_{\lambda}\right)$ be the $\lambda$-blow up map. Let $h_{\lambda}$ : $\mathbf{B}(1) \rightarrow\left(Y, d_{\lambda}\right)$ be a map which is harmonic with respect to the Euclidean domain metric and with the boundary condition $\left.h_{\lambda}\right|_{\partial \mathbf{B}(1)}=\left.f_{\lambda}\right|_{\partial \mathbf{B}(1)}$. Then

$$
(1-c \lambda){ }^{\delta} E^{f_{\lambda}} \leq{ }^{g_{\lambda}} E^{f_{\lambda}} \leq(1+c \lambda){ }^{\delta} E^{f_{\lambda}}
$$

and

$$
(1-c \lambda){ }^{\delta} E^{h_{\lambda}} \leq{ }^{g_{\lambda}} E^{h_{\lambda}} \leq(1+c \lambda){ }^{\delta} E^{h_{\lambda}} .
$$

Proof. By inequality (6.3), we have

$$
\begin{aligned}
(1-c \lambda) \sum_{i, j=1}^{n} \delta^{i j} \frac{\partial f_{\lambda}}{\partial x_{i}} \cdot \frac{\partial f_{\lambda}}{\partial x_{j}} & \leq \sum_{i, j=1}^{n}\left(g_{\lambda}\right)^{i j} \frac{\partial f_{\lambda}}{\partial x_{i}} \cdot \frac{\partial f_{\lambda}}{\partial x_{j}} \\
& \leq(1+c \lambda) \sum_{i, j=1}^{n} \delta^{i j} \frac{\partial f_{\lambda}}{\partial x_{i}} \cdot \frac{\partial f_{\lambda}}{\partial x_{j}}
\end{aligned}
$$

and

$$
\begin{aligned}
(1-c \lambda) \sum_{i, j=1}^{n} \delta^{i j} \frac{\partial h_{\lambda}}{\partial x_{i}} \cdot \frac{\partial h_{\lambda}}{\partial x_{j}} & \leq \sum_{i, j=1}^{n}\left(g_{\lambda}\right)^{i j} \frac{\partial h_{\lambda}}{\partial x_{i}} \cdot \frac{\partial h_{\lambda}}{\partial x_{j}} \\
& \leq(1+c \lambda) \sum_{i, j=1}^{n} \delta^{i j} \frac{\partial h_{\lambda}}{\partial x_{i}} \cdot \frac{\partial h_{\lambda}}{\partial x_{j}}
\end{aligned}
$$

The assertion follows immediately.

In particular, Lemma 6.2 and (6.1) imply that

$$
{ }^{\delta} E^{h_{\lambda}} \leq{ }^{\delta} E^{f_{\lambda}} \leq \frac{1}{1-c \lambda}{ }^{g_{\lambda}} E^{f_{\lambda}} \leq \frac{2 \alpha}{1-c \lambda} .
$$

Thus, Proposition 5.1 and Theorem 4.2 imply that there exists a subsequence of $\lambda_{k}$ (which we will still denote $\lambda_{k}$ by an abuse of notation) and an NPC 
space $\left(\bar{Y}_{*}, \bar{d}_{*}\right)$ so that $h_{\lambda_{k}}$ converge locally uniformly in the pullback sense to $h_{*}: \mathbf{B}(1) \rightarrow\left(\bar{Y}_{*}, \bar{d}_{*}\right)$. Set $h_{k}:=h_{\lambda_{k}}, f_{k}:=f_{\lambda_{k}}$ and $g_{k}=g_{\lambda_{k}}$. Furthermore, let $d_{k}(x, y)=d_{\lambda_{k}}\left(f_{k}(x), f_{k}(y)\right)$ and $\bar{d}_{k}(x, y)=d_{\lambda_{k}}\left(h_{k}(x), h_{k}(y)\right)$. Then in any compactly contained subset of $\mathbf{B}(1) \times \mathbf{B}(1), d_{k}, \bar{d}_{k}$ converge uniformly to (the restriction to $\mathbf{B}(1)=\Omega_{0}$ of) $d_{*}, \bar{d}_{*}$ respectively.

Proposition 6.1. Under the notation above, the pseudodistance functions $d_{*}$ and $\bar{d}_{*}$ above are equal. Consequently, $f_{*}=h_{*}$ and $h_{k}$ (which are harmonic maps with respect to the Euclidean metric) converge locally uniformly in the pullback sense to $f_{*}$.

Proof. By the repeated use of the triangle inequality,

$$
\left|d_{k}(x, y)-\bar{d}_{k}(x, y)\right| \leq d_{\lambda_{k}}\left(f_{k}(x), h_{k}(x)\right)+d_{\lambda_{k}}\left(f_{k}(y), h_{k}(y)\right) .
$$

Therefore, for any $r<1$, the Lebesgue dominated convergence theorem and the Poincaré inequality (cf. Theorem 2.6) imply

$$
\begin{aligned}
& \int_{\mathbf{B}(r)} \int_{\mathbf{B}(r)}\left|d_{*}(x, y)-\bar{d}_{*}(x, y)\right|^{2} d \mu(x) d \mu(y) \\
& =\lim _{k \rightarrow 0} \int_{\mathbf{B}(r)} \int_{\mathbf{B}(r)}\left|d_{k}(x, y)-\bar{d}_{k}(x, y)\right|^{2} d \mu(x) d \mu(y) \\
& \leq 4 \operatorname{vol}(\mathbf{B}(r)) \lim _{k \rightarrow 0} \int_{\mathbf{B}(r)} d_{\lambda_{k}}^{2}\left(f_{k}(x), h_{k}(x)\right) d \mu(x) \\
& \leq 4 C \lim _{k \rightarrow 0} \int_{\mathbf{B}(r)}\left|\nabla d_{\lambda_{k}}^{2}\left(f_{k}(x), h_{k}(x)\right)\right| d \mu(x) .
\end{aligned}
$$

Equations (6.4) and (6.5) imply

$$
{ }^{\delta} E^{f_{\lambda}} \leq \frac{1}{1-c \lambda}{ }^{g_{\lambda}} E^{f_{\lambda}} \leq \frac{1}{1-c \lambda}{ }^{g_{\lambda}} E^{h_{\lambda}} \leq \frac{1+c \lambda}{1-c \lambda}{ }^{\delta} E^{h_{\lambda}} .
$$

Therefore, if we let $w=\frac{1}{2} f_{\lambda}+\frac{1}{2} h_{\lambda}$,

$$
\begin{aligned}
2{ }^{\delta} E^{w} & \leq{ }^{\delta} E^{f_{\lambda}}+{ }^{\delta} E^{h_{\lambda}}-\frac{1}{2} \int_{\mathbf{B}(r)}\left|\nabla d_{\lambda}^{2}\left(f_{\lambda}, h_{\lambda}\right)\right| d \mu \\
& =2{ }^{\delta} E^{h_{\lambda}}+O(\lambda)-\frac{1}{2} \int_{\mathbf{B}(r)}\left|\nabla d_{\lambda}^{2}\left(f_{\lambda}, h_{\lambda}\right)\right| d \mu
\end{aligned}
$$


by Equation (2.2iv) of [11]. Since ${ }^{\delta} E^{h_{\lambda}} \leq{ }^{\delta} E^{w}$, this in turn implies

$$
\int_{\mathbf{B}(r)}\left|\nabla d_{\lambda}^{2}\left(f_{\lambda}, h_{\lambda}\right)\right| d \mu \rightarrow 0
$$

as $\lambda \rightarrow 0$. This, combined with Equation (6.9) the continuity of $d_{*}$ and $\bar{d}_{*}$, implies that $d_{*}(x, y)=\bar{d}_{*}(x, y)$ which in turn implies that $\left(Y_{*}, d_{*}\right)=\left(\bar{Y}_{*}, \bar{d}_{*}\right)$ and $h_{*}=f_{*}$.

Lemma 6.3. Assuming that the directional energies of $h_{k}$ converge to those of $f_{*}$, the tangent map $f_{*}: \mathbf{B}(1) \rightarrow Y_{*}$ is homogenous of order $\alpha$ where $\alpha$ is the order of $f$ at 0 , i.e.,

$$
d_{*}\left(f_{*}(x), f_{*}(0)\right)=|x|^{\alpha} d_{*}\left(f_{*}\left(\frac{x}{|x|}\right), f_{*}(0)\right)
$$

and the image of $t \mapsto f_{*}(t x), 0 \leq t \leq 1$, is a geodesic.

Proof. For notational simplicity in this proof we will let $E={ }^{\delta} E$ and $I={ }^{\delta} I$. Using (3.6) and (3.11) with $f$ replaced by $h_{k}$, noting that the remainder in (3.6) is 0 because the domain is Euclidean, and using the convergence of $h_{k}$ and its directional energies to $f_{*}$ and its directional energies, we have

$$
\left(E^{f_{*}}(\sigma)\right)^{\prime}=2 \int_{\partial \mathbf{B}(\sigma)}\left|\frac{\partial f_{*}}{\partial r}\right|^{2} d \Sigma
$$

and

$$
2 E^{f_{*}}(\sigma) \leq \int_{\partial \mathbf{B}(\sigma)} d\left(f_{*}, f_{*}(0)\right) \frac{\partial}{\partial r} d\left(f_{*}, f_{*}(0)\right) d \Sigma .
$$

We next claim

$$
\begin{aligned}
\left(\log \left(\frac{\sigma E^{f_{*}}(\sigma)}{I^{f_{*}}(\sigma)}\right)\right)^{\prime} \\
\geq \frac{2}{E^{f_{*}}(\sigma) I^{f_{*}}(\sigma)}\left[\left(\int_{\mathbf{B}(\sigma)} d^{2}\left(f_{*}, f_{*}(0)\right) d \Sigma\right)\left(\int_{\mathbf{B}(\sigma)}\left|\frac{\partial f_{*}}{\partial r}\right|^{2} d \Sigma\right)\right. \\
\left.\quad-\int_{\partial \mathbf{B}(\sigma)}\left(d\left(f_{*}, f_{*}(0)\right) \frac{\partial}{\partial r} d\left(f_{*}, f_{*}(0)\right) d \Sigma\right)^{2}\right] \geq 0 .
\end{aligned}
$$

This follows from (3.9), (3.14) and (3.19) applied to the harmonic map $h_{k}$ (without the error term due to the fact that $h_{k}$ is harmonic for the Euclidean domain metric) and the assumption that the directional energies 
of $h_{k}$ converge to those of $f_{*}$. On the other hand, our assumption on the convergence of directional energies implies that

$$
\begin{aligned}
\frac{\sigma E^{f_{*}}(\sigma)}{I^{f_{*}}(\sigma)} & =\lim _{k \rightarrow \infty} \frac{\sigma E_{g_{k}}^{f_{k}}(\sigma)}{I_{g_{k}}^{f_{k}}(\sigma)} \\
& =\lim _{k \rightarrow \infty} \frac{\sigma \mu_{\lambda_{k}}^{-2} \lambda_{k}^{2-n}{ }^{g} E^{f}\left(\lambda_{k} \sigma\right)}{\mu_{\lambda_{k}}^{-2} \lambda_{k}^{1-n} I^{f}\left(\lambda_{k} \sigma\right)} \\
& =\lim _{k \rightarrow \infty} \frac{\sigma \lambda_{k}{ }^{g} E^{f}\left(\lambda_{k} \sigma\right)}{{ }^{g} I^{f}\left(\lambda_{k} \sigma\right)} \\
& =\alpha .
\end{aligned}
$$

Thus,

$$
\begin{aligned}
& 0=\left(\log \left(\frac{\sigma E^{f_{*}}(\sigma)}{I^{f_{*}}(\sigma)}\right)\right)^{\prime} \\
&=\frac{2}{E^{f_{*}}(\sigma) I^{f_{*}}(\sigma)}\left[\left(\int_{\mathbf{B}(\sigma)} d^{2}\left(f_{*}, f_{*}(0)\right) d \Sigma\right)\left(\int_{\mathbf{B}(\sigma)}\left|\frac{\partial f_{*}}{\partial r}\right|^{2} d \Sigma\right)\right. \\
&\left.-\int_{\partial \mathbf{B}(\sigma)}\left(d\left(f_{*}, f_{*}(0)\right) \frac{\partial}{\partial r} d\left(f_{*}, f_{*}(0)\right) d \Sigma\right)^{2}\right] .
\end{aligned}
$$

Hence,

$$
\frac{\partial}{\partial r} d\left(f_{*}, f_{*}(0)\right)=\left|\frac{\partial f_{*}}{\partial r}\right| \text { a.e. }
$$

and

$$
2 \int_{\mathbf{B}(\sigma)}\left|\nabla f_{*}\right|^{2} d \mu=\int_{\partial \mathbf{B}(\sigma)} \frac{\partial}{\partial r}\left(d^{2}\left(f_{*}, f_{*}(0)\right)\right) d \Sigma .
$$

We can now follow the proof of Proposition 3.1 [9] to show the homogeneity of $f_{*}$.

Lemma 6.4. Let $f_{*}: \mathbf{B}(1) \rightarrow\left(Y_{*}, d_{*}\right)$ be a homogeneous map of order $\alpha$. (See definition of homogeneity in Lemma 6.3.) Then there exist a metric space $(C, \hat{d})$ and a map $\hat{f}_{*}: \mathbf{B}(1) \rightarrow C$ so that the energy density of $f_{*}$ is equal to that of $\hat{f}_{*}$ and for every $x, y \in \mathbf{B}(r)$ and $x^{\prime}=t x, y^{\prime}=t y$, we have

$$
\hat{d}\left(\hat{f}_{*}\left(x^{\prime}\right), \hat{f}_{*}\left(y^{\prime}\right)\right)=t^{\alpha} \hat{d}\left(\hat{f}_{*}(x), \hat{f}_{*}(y)\right) .
$$

Proof. Let $C$ be the disjoint union of geodesics from $f_{*}(0)$ to $f_{*}(x)$ for each $x \in \partial \mathbf{B}(1)$ with $f_{*}(0)$ identified. We define a distance function $\hat{d}$ on $C$ in 
the following way. Let $P, Q \in C$ and suppose that $P$ (resp. $Q$ ) is the point on the geodesic $\gamma\left(\right.$ resp. $\sigma$ ) from $f_{*}(0)$ to $f_{*}(x)$ (resp. $f_{*}(y)$ ) at a distance $r$ (resp. $s$ ) from $f_{*}(0)$, where $x, y \in \partial \mathbf{B}(1)$. We first define the angle $\theta$ between $\gamma$ and $\sigma$ by

$$
\cos \theta=\frac{d_{*}^{2}\left(f_{*}(0), f_{*}(x)\right)+d_{*}^{2}\left(f_{*}(0), f_{*}(y)\right)-d_{*}^{2}\left(f_{*}(x), f_{*}(y)\right)}{2 d_{*}\left(f_{*}(0), f_{*}(x)\right) d_{*}\left(f_{*}(0), f_{*}(y)\right)}
$$

and set

$$
\begin{gathered}
\hat{d}^{2}(P, Q)=r^{2}+s^{2}-2 r s \cos \theta, \\
\hat{f}_{*}(x)=f_{*}(x) .
\end{gathered}
$$

By definition of $\hat{d}$, we see that (6.11) holds. Therefore,

$$
\hat{d}\left(\hat{f}_{*}(x), \hat{f}_{*}(y)\right)=d_{*}\left(f_{*}(x), f_{*}(y)\right)
$$

whenever $x, y$ lie on the same geodesic from 0 or whenever $x, y \in \partial \mathbf{B}(1)$. Therefore, for any $x \in \partial \mathbf{B}(r)$ and any vector $V$ normal to $\partial \mathbf{B}(r)$,

$$
\left|f_{*}(V)\right|^{2}(x)=\left|\hat{f}_{*}(V)\right|^{2}(x) .
$$

Furthermore, the same holds for any $x \in \partial \mathbf{B}(1)$ and $V$ tangential to $\partial \mathbf{B}(1)$. For a.e. $x=(r, \theta) \in \mathbf{B}(r)$ and a vector $V$ tangential to $\partial \mathbf{B}(r)$, [11, Lemma 1.9.4] implies that

$$
\begin{aligned}
\left|\hat{f}_{*}(V)\right|^{2}(r, \theta) & =\lim _{\epsilon \rightarrow 0} \frac{\hat{d}^{2}\left(\hat{f}_{*}(r, \theta), \hat{f}_{*}(r, \theta+\epsilon V)\right)}{\epsilon^{2}} \\
& =r^{2 \alpha} \lim _{\epsilon \rightarrow 0} \frac{\hat{d}^{2}\left(\hat{f}_{*}(1, \theta), \hat{f}_{*}(1, \theta+\epsilon V)\right)}{\epsilon^{2}} \\
& =r^{2 \alpha} \lim _{\epsilon \rightarrow 0} \frac{d_{*}^{2}\left(f_{*}(1, \theta), f_{*}(1, \theta+\epsilon V)\right)}{\epsilon^{2}} \\
& =r^{2 \alpha}\left|f_{*}(V)\right|^{2}(1, \theta) \\
& =\left|f_{*}(V)\right|^{2}(r, \theta),
\end{aligned}
$$

where $(r, \theta)$ is the polar coordinates of $x$ and $\epsilon \mapsto \theta+\epsilon V$ is the flow along $\partial \mathbf{B}(r)$ defined by $V$.

\section{Harmonic maps from a flat domain}

Let $\mathbf{B}$ be a dimension- $n$ codimension- $\nu$ local model with wedges $W_{k} \subset \mathbf{R}^{n}$, $k=1, \ldots, N$. Recall that the coordinates $\left(x^{1}, \ldots, x^{n}\right)$ of $\mathbf{R}^{n}$ are arranged 
so that $D$ is given by $x^{n-\nu+1}=\cdots=x^{n}=0$. In this section, we show that harmonic maps $h:(\mathbf{B}(r), \delta) \rightarrow(Y, d)$ are Lipschitz in the direction parallel to $D(r)=D \cap \mathbf{B}(r)$.

Lemma 7.1. Let $\phi: \mathbf{B}(1) \rightarrow \mathbf{R}$ be a non-negative $L^{2}$ function. Suppose that for $x \in \mathbf{B}(r)$, there exists $\hat{C}>0$ so that

$$
\frac{1}{\sigma^{n}} \int_{B_{x}(\sigma)} \phi d \mu \leq \frac{\hat{C}}{\rho^{n}} \int_{B_{x}(\rho)} \phi d \mu, 0<\sigma \leq \rho \leq \min \{R(x), 1-r\} .
$$

There exist $C>0$ and $R \in(0,1-r)$ depending only on $r$ and $\mathbf{B}(r)$ such that

$$
\frac{1}{\sigma^{n}} \int_{B_{x}(\sigma)} \phi d \mu \leq C \int_{B_{x}(R)} \phi d \mu, \forall x \in \mathbf{B}(r), \sigma \in(0, R] .
$$

Proof. The result follows from the same argument contained in the proofs of Corollary 4.2 and Proposition 4.3 (with $\phi$ replacing $|\nabla f|^{2}, \lambda=1$ and $\beta=1)$.

Lemma 7.2. Let $h:(\mathbf{B}(1), \delta) \rightarrow(Y, d)$ be a harmonic map. Let $V$ be a unit vector parallel to $D(r)$ and let $H(x)=h(x+\epsilon V)$ for $0<\epsilon<<1$. Then

$$
0 \leq-\int_{\mathbf{B}(1)} \nabla \eta \cdot \nabla d^{2}(h, H) d \mu
$$

for $\eta \in C_{c}^{\infty}(\mathbf{B}(1-\epsilon))$.

Proof. Define a map $h_{\eta}: \mathbf{B}(1-\epsilon) \rightarrow \mathbf{R}$ by setting

$$
h_{\eta}(x)=(1-\eta(x)) h(x)+\eta(x) H(x) .
$$

Here, $(1-t) P+t Q$ for $P, Q \in Y$ denotes the point on the unique geodesic between $P$ and $Q$ at a distance $t d(P, Q)$ from $P$ and $(1-t) d(P, Q)$ from $Q$. Since $\operatorname{spt}(\eta) \subset \mathbf{B}(1-\epsilon)$, we see that

$$
\left.h_{\eta}\right|_{\partial \mathbf{B}(1-\epsilon)}=\left.h\right|_{\partial \mathbf{B}(1-\epsilon)}
$$

and

$$
\left.h_{1-\eta}\right|_{\partial \mathbf{B}(1-\epsilon)}=\left.H\right|_{\partial \mathbf{B}(1-\epsilon)} .
$$


By following the proofs of Lemmas 2.4.1 and 2.4.2 of [11], we see that $h_{\eta}, h_{1-\eta} \in W^{1,2}(\mathbf{B}(r))$, and on each wedge $W_{k}, k=1, \ldots, N$, we have

$$
\begin{aligned}
& \int_{\mathbf{B}(1-\epsilon) \cap W_{k}}\left|\nabla h_{\eta}\right|^{2} d \mu+\int_{\mathbf{B}(1-\epsilon) \cap W_{k}}\left|\nabla h_{1-\eta}\right|^{2} d \mu \\
& \leq \int_{\mathbf{B}(1-\epsilon) \cap W_{k}}|\nabla h|^{2} d \mu+\int_{\mathbf{B}(1-\epsilon) \cap W_{k}}|\nabla H|^{2} d \mu \\
& \quad-2 \int_{\mathbf{B}(1-\epsilon) \cap W_{k}} \nabla \eta \cdot \nabla d^{2}(h, H) d \mu+\int_{\mathbf{B}(1-\epsilon) \cap W_{k}} Q(\eta, \nabla \eta) d \mu,
\end{aligned}
$$

where $Q(\eta, \nabla \eta)$ consists of integrable terms which are quadratic in $\eta$ and $\nabla \eta$. Taking the sum over $k=1, \ldots, l$ and noting that the harmonicity of $h$ and $H$ implies

$$
\int_{\mathbf{B}(1-\epsilon)}|\nabla h|^{2} d \mu \leq \int_{\mathbf{B}(1-\epsilon)}\left|\nabla h_{\eta}\right|^{2} d \mu
$$

and

$$
\int_{\mathbf{B}(1-\epsilon)}|\nabla H|^{2} d \mu \leq \int_{\mathbf{B}(1-\epsilon)}\left|\nabla h_{1-\eta}\right|^{2} d \mu
$$

we deduce

$$
0 \leq-2 \int_{\mathbf{B}(1-\epsilon)} \nabla \eta \cdot \nabla d^{2}(h, H) d \mu+\int_{\mathbf{B}(1-\epsilon)} Q(\eta, \nabla \eta) d \mu .
$$

By replacing $\eta$ by $t \eta$, dividing by $t$ and letting $t \rightarrow 0$, we obtain (7.1).

Lemma 7.3. Let $h:(\mathbf{B}(1), \delta) \rightarrow(Y, d)$ be a harmonic map and let $V$ be a unit vector parallel to $D(1)$. For $r \in(0,1)$ and $x \in \mathbf{B}(r)$, there exists a constant $C$ depending only on $r$ and $\mathbf{B}(r)$ so that

$$
\left|h_{*}(V)\right|^{2}(x) \leq C^{\delta} E^{h}
$$

Proof. Let $H$ be as in the proof of Lemma 7.2. For $x \in \mathbf{B}(1)$ and $\sigma \in$ $(0, R(x))$, let $\eta$ approximate the characteristic function of $B_{x}(\sigma)$ in $(7.1)$ to obtain

$$
\int_{\partial B_{x}(\sigma)} \frac{\partial}{\partial r} d^{2}(h, H) d \Sigma \geq 0
$$

Let

$$
J_{x}(\sigma)=\int_{\partial B_{x}(\sigma)} d^{2}(h, H) d \Sigma \text { and } K_{x}(\sigma)=\int_{B_{x}(\sigma)} d^{2}(h, H) d \mu
$$


Then

$J_{x}^{\prime}(\sigma)=\int_{\partial B_{x}(\sigma)} \frac{\partial}{\partial r} d^{2}(h, H) d \Sigma+\frac{n-1}{\sigma} J_{x}(\sigma) \geq \frac{n-1}{\sigma} J_{x}(\sigma), \forall \sigma \in(0, R(x))$.

This implies that

$$
\left(\frac{J_{x}(\sigma)}{\sigma^{n-1}}\right)^{\prime} \geq 0
$$

and hence

$$
J_{x}(\tau) \leq \frac{J_{x}(\sigma)}{\sigma^{n-1}} \tau^{n-1}, \quad 0<\tau \leq \sigma \leq R(x) .
$$

Now integrate the above inequality from $\tau=0$ to $\sigma$ to obtain

$$
K_{x}(\sigma) \leq \frac{\sigma J_{x}(\sigma)}{n}=\frac{\sigma K_{x}^{\prime}(\sigma)}{n}
$$

Thus,

$$
\left(\frac{K_{x}(\sigma)}{\sigma^{n}}\right)^{\prime}=\frac{1}{\sigma^{n}}\left(K_{x}^{\prime}(\sigma)-\frac{n K_{x}(\sigma)}{\sigma}\right) \geq 0
$$

This implies $\sigma \mapsto \frac{K_{x}(\sigma)}{\sigma^{n}}$ is non-decreasing for $\sigma \in(0, R(x))$ and hence

$$
\frac{K_{x}(\sigma)}{\sigma^{n}} \leq \frac{K_{x}(\rho)}{\rho^{n}}, 0<\sigma \leq \rho \leq R(x) .
$$

By Lemma 7.1, there exists $C>0$ and $R>0$ such that

$$
\frac{K_{x}(\sigma)}{\sigma^{n}} \leq C K_{x}(R), \forall x \in \mathbf{B}(r)
$$

Fix $x \in \mathbf{B}(r)$ and let $\sigma \rightarrow 0$ to obtain

$$
d^{2}(h(x), H(x)) \leq C \int_{B_{x}(R)} d^{2}(h, H) d \mu .
$$

Divide by $\epsilon^{2}$ and let $\epsilon \rightarrow 0$ to obtain

$$
\left|h_{*}(V)\right|^{2}(x) \leq C \int_{B_{x}(R)}\left|h_{*}(V)\right|^{2} d \mu \leq C^{\delta} E^{h}
$$


Lemma 7.4. Let $h:(\mathbf{B}(1), \delta) \rightarrow(Y, d)$ be a harmonic map and $r \in(0,1)$. If $x, y$ are a pair of points in a wedge of $\mathbf{B}(r)$ equidistant to $D(r)$, then

$$
d(h(x), h(y)) \leq L|x-y|
$$

for some constant $L$ depending only on ${ }^{\delta} E^{h}, r$ and $\mathbf{B}(1)$.

Proof. Let $\gamma:[0,1] \rightarrow(Y, d)$ be a constant speed parameterization of the line between $x$ and $y$. Then by Lemma 7.3 ,

$$
d(h(x), h(y)) \leq \int_{0}^{1}\left|h_{*}\left(\gamma^{\prime}(t)\right)\right| d t \leq \sqrt{C^{\prime} \delta E^{h}}|x-y| .
$$

\section{Lipschitz regularity}

\subsection{At a regular point}

In this subsection, we use the results of Section 7 to give a new proof of the Lipschitz regularity of Korevaar-Schoen [11] and generalize their result for Lipschitz domain metrics. Recall that a dimension- $n$, codimension-0 local model is $\mathbf{B}=\mathbf{R}^{n}$.

Lemma 8.1. Let $\mathbf{B}$ be a dimension-n, codimension-0 local model, $g$ a normalized Lipschitz metric defined on $\mathbf{B}(r),(Y, d)$ an NPC space and $f$ : $(\mathbf{B}(r), g) \rightarrow(Y, d)$ a harmonic map. Then the order $\alpha$ of $f$ at 0 is $\geq 1$.

Proof. By Proposition 6.1, a tangent map $f_{*}$ of $f$ is a locally uniform limit of a sequence of harmonic maps $h_{k}$ from a Euclidean unit ball $\mathbf{B}(1)$. The regularity result of [9] implies that $h_{k}$ is locally Lipschitz with the local Lipschitz bound depending on ${ }^{\delta} E^{h_{k}}$ and the distance to $\partial \mathbf{B}(1)$. Hence, so is $f_{*}$. By Korevaar and Schoen [12, Theorem 3.11], the energy densities of $h_{k}$ converge to those of $f_{*}$. By Lemma $6.3, f_{*}$ is a homogeneous map of order $\alpha$. The homogeneity and the Lipschitz continuity of $f_{*}$ implies $\alpha \geq 1$.

Theorem 8.1. Let $\mathbf{B}$ be a dimension-n, codimension-0 local model, $g$ a Lipschitz Riemannian metric on $\mathbf{B}(r),(Y, d)$ an $N P C$ space and $f:(\mathbf{B}(r), g) \rightarrow$ $(Y, d)$ a harmonic map. Then $f$ is Lipschitz continuous in $\mathbf{B}(\varrho r)$ with the Lipschitz constant depending on $\varrho \in(0,1),(\mathbf{B}(r), g)$ and the total energy $E^{f}$ of $f$. 
Proof. For each $x \in \mathbf{B}(1)$, the normalized map $f \circ L_{x}$ (cf. Proposition 2.1) has order $\geq 1$ at 0 by Lemma 8.1. Thus, the order of $f$ at $x$ is $\geq 1$. The result now follows from Theorem 4.1.

\subsection{At a codimension-1 singular point}

Throughout this subsection $\mathbf{B}$ is a dimension- $n$, codimension-1 local model with wedges half spaces given by $x^{n} \geq 0$ and lower dimensional stratum $D$ a hyperplane given by the equation $x^{n}=0$. We first prove some properties of harmonic maps from this local model equipped with the Euclidean metric $\delta$.

Lemma 8.2. Let $h:(\mathbf{B}(1), \delta) \rightarrow(Y, d)$ be a harmonic map. For every $\beta, r \in$ $(0,1)$, there exists $B$ depending only on $\beta, r, \mathbf{B}(1)$ and ${ }^{\delta} E^{h}$ so that

$$
d(h(x), h(y)) \leq B|x-y|^{\beta}
$$

for every $x, y \in \mathbf{B}(r)$.

Proof. By Lemma 7.4, $h$ is Lipschitz when restricted to $D\left(t_{0}\right), t_{0}=\frac{r+1}{2}$. Thus Hölder regularity of $h$ restricted to a wedge $W$ with any Hölder exponent $\beta \in(0,1)$ follows from the boundary regularity result of Serbinowski [15] where the Hölder constant $B$ is only depending on the choice of $\beta, r$ and the total energy of the map $h$.

The next lemma gives an estimate of the energy decay of harmonic maps along an $\epsilon$-neighborhood.

Lemma 8.3. Let $h_{k}$ be defined as in Section 6 (see the paragraph preceeding Proposition 6.1) and fix $R \in(0,1)$. Set $D_{\epsilon}(r)$ to be the $\epsilon$-neighborhood of $D(r)$ in $\mathbf{B}(r)$, i.e.,

$$
D_{\epsilon}(r)=\cup\left\{x=\left(x_{1}, \ldots, x_{n}\right) \in W: x_{n} \leq \epsilon\right\} \cap \mathbf{B}(r),
$$

where the union is over the wedges $W$ containing $D$. Then for any $r \in(0, R)$, there exist constants $C, \kappa>0, k_{0}$ sufficiently large and $\epsilon_{0}>0$ sufficiently small (depending only on $R$ ) so that

$$
{ }^{\delta} E^{h_{k}}\left[D_{\epsilon}(r)\right] \leq C \epsilon^{\kappa}, \forall k>k_{0}, \epsilon<\epsilon_{0}
$$


Proof. Let $B_{x}(r)$ be a ball of radius $r$ centered at $x$. We will use the notation,

$$
E_{x}^{h}(r)={ }^{\delta} E_{x}^{h}(r)=\int_{B_{x}(r)}|\nabla h|^{2} d \mu
$$

and

$$
I_{x}^{h}(r)={ }^{\delta} I_{x}^{h}(r)=\int_{\partial B_{x}(r)} d^{2}(h, h(x)) d \Sigma
$$

for any map $h: \mathbf{B}(r) \rightarrow(Y, d)$. Let $r_{0}:=1-R$. By Lemma $6.1, f_{*}$ is a nonconstant, continuous map and hence there exists $c_{1}>0$ so that

$$
I_{x}^{f_{*}}\left(r_{0}\right) \geq 2 c_{1}, \forall x \in D(R)
$$

Thus, by the local uniform convergence, there exists $k_{0}$ so that

$$
I_{x}^{h_{k}}\left(r_{0}\right) \geq c_{1}, \forall x \in D(R), k>k_{0}
$$

We may assume we have chosen $k$ sufficiently large so that $\lambda_{k_{0}} \in\left(0, \frac{1}{N c}\right)$. By (6.8),

$$
E_{x}^{h_{k}}\left(r_{0}\right) \leq E^{h_{k}}(1) \leq E^{f_{k}}(1) \leq \frac{2 \alpha}{1-c \lambda_{k_{0}}}, \forall x \in D(R), k>k_{0}
$$

Thus,

$$
\frac{r_{0} E_{x}^{h_{k}}\left(r_{0}\right)}{I_{x}^{h_{k}}\left(r_{0}\right)} \leq \frac{2 r_{0} \alpha}{\left(1-c \lambda_{k_{0}}\right) c_{1}}=: c_{2}, \forall x \in D(R), k>k_{0} .
$$

By Corollary 3.2,

$$
\frac{\epsilon E_{x}^{h_{k}}(\epsilon)}{I_{x}^{h_{k}}(\epsilon)} \leq c_{3}, \forall x \in D(R), k>k_{0}, \epsilon \leq r_{0}
$$

with $c_{3}$ depending on $c_{2}$. By Lemma 8.2,

$$
E_{x}^{h_{k}}(\epsilon) \leq \frac{c_{3} I_{x}^{h_{k}}(\epsilon)}{\epsilon} \leq \frac{c_{3} B^{2} \epsilon^{2 \beta+n-1}}{\epsilon}=c_{3} B^{2} \epsilon^{2 \beta+n-2}, \forall x \in D(R), k>k_{0} .
$$

Here, we have choosen $\beta \in(1 / 2,1)$. Since $D_{\epsilon}(r)$ can be covered by $\left(\frac{c_{4}}{\epsilon}\right)^{n-1}$ number of $(2 \epsilon)$-balls centered at points in $D(r)$ where $c_{4}$ is 
independent of $\epsilon$,

$$
\begin{aligned}
E^{h_{k}}\left[D_{\epsilon}(r)\right] & \leq c_{3} B^{2}(2 \epsilon)^{2 \beta+n-2} \times\left(\frac{c_{4}}{\epsilon}\right)^{n-1} \\
& =: c_{5} \epsilon^{2 \beta-1}
\end{aligned}
$$

The result follows from the fact that the choice of $\beta$ implies $2 \beta-1>0$.

Lemma 8.4. Let $h_{k}, f_{k}$ and $h_{*}=f_{*}$ be defined as in Section 6. For $r \in$ $(0,1)$,

$$
\lim _{k \rightarrow 0}{ }^{\delta} E^{h_{k}}(r)={ }^{\delta} E^{h_{*}}(r)
$$

and

$$
\lim _{k \rightarrow \infty}{ }^{g_{k}} E^{f_{k}}(r)={ }^{\delta} E^{f_{*}}(r),
$$

and the directional energies of $f_{k}$, and $h_{k}$ converge to that of $f_{*}$.

Proof. Again in this proof we will denote ${ }^{\delta} E=E$. By the regularity result of harmonic maps from smooth domains [11, Theorem 2.4.6] or Theorem 8.1, $h_{k}$ is uniformly Lipschitz in $\mathbf{B}\left(\frac{1+r}{2}\right)-D_{\frac{\epsilon}{2}}\left(\frac{1+r}{2}\right)$ for $r \in(0,1)$. First, we note that

$$
\lim _{k \rightarrow \infty} E^{h_{k}}\left[\mathbf{B}(r)-D_{\epsilon}(r)\right]=E^{f_{*}}\left[\mathbf{B}(r)-D_{\epsilon}(r)\right] .
$$

This follows from [11, Theorem 3.11]. By Lemma 8.3,

$$
E^{h_{k}}\left[D_{\epsilon}(r)\right] \leq C \epsilon^{\kappa}
$$

for any $\epsilon$ sufficiently small. Thus,

$$
\begin{aligned}
\limsup _{k \rightarrow \infty} E^{h_{k}}(r)-C \epsilon^{\kappa} & \leq \limsup _{k \rightarrow \infty} E^{h_{*}}\left[\mathbf{B}(r)-D_{\epsilon}(r)\right] \\
& =E^{f_{*}}\left[\mathbf{B}(r)-D_{\epsilon}(r)\right] \\
& \leq E^{f_{*}}(r)
\end{aligned}
$$

By lower semicontinuity of energy,

$$
E^{f_{*}}(r) \leq \liminf _{k \rightarrow \infty} E^{h_{k}}(r) \leq \limsup _{k \rightarrow \infty} E^{h_{k}}(r) \leq E^{f_{*}}(r)+C \epsilon^{\kappa} .
$$


Since $\epsilon>0$ can be made arbitrarily small, this proves (8.1). To prove (8.2), we see that

$$
\begin{aligned}
E^{f_{*}}(r) & \leq \liminf _{k \rightarrow \infty}\left(1-c \lambda_{k}\right) E^{f_{k}}(r) \\
& \leq \liminf _{k \rightarrow \infty}{ }^{g_{k}} E^{f_{k}}(r) \\
& \leq \liminf _{k \rightarrow \infty}{ }^{g_{k}} E^{h_{k}}(r) \\
& \leq \limsup _{k \rightarrow \infty}\left(1+c \lambda_{k}\right) E^{h_{k}}(r) \\
& =E^{h_{*}}(r) \\
& =E^{f_{*}}(r) .
\end{aligned}
$$

Here, the last line follows from the fact that $f_{*}=h_{*}$ by Proposition 6.1. Since there is no loss of total energy, we see that the directional energies converge by using the lower semicontinuity.

Lemma 8.5. Let $g$ be a normalized Lipschitz metric on $\mathbf{B}(r)$ and $f$ : $(\mathbf{B}(r), g) \rightarrow(Y, d)$ a harmonic map. Then its tangent map $f_{*}: \mathbf{B}(1) \rightarrow Y_{*}$ is homogeneous of order $\alpha$ where $\alpha$ is the order of $f$ at 0 .

Proof. Follows immediately from Lemmas 6.3 and 8.4.

Lemma 8.6. Let $g$ be a normalized Lipschitz metric on $\mathbf{B}(r), f:(\mathbf{B}(r), g)$ $\rightarrow(Y, d)$ a harmonic map and $f_{*}: \mathbf{B}(1) \rightarrow Y_{*}$ its tangent map. For every $\beta, r^{\prime} \in(0,1)$, there exists $B$ so that

$$
d_{*}\left(f_{*}(x), f_{*}(y)\right) \leq B|x-y|^{\beta}
$$

for all $x, y \in \mathbf{B}\left(r^{\prime}\right)$ and $B$ is only depending on the choice of $\beta, r^{\prime}, \mathbf{B}(1)$ and the total energy of $f_{*}$.

Proof. First, note that $h_{k}$ converges to $f_{*}$ uniformly by Proposition 6.1. Next, note that the energy of $h_{k}$ converges to that of $f_{*}$ by Lemma 8.4. Thus, the result follows from Lemma 8.2.

Lemma 8.7. Let $g$ be a normalized Lipschitz Riemannian metric on $\mathbf{B}(r)$ and $f:(\mathbf{B}(r), g) \rightarrow(Y, d)$ a harmonic map. Then the order $\alpha$ of $f$ at 0 is $\geq 1$. 
Proof. Since $f_{*}$ is homogeneous of degree $\alpha$,

$$
d_{*}\left(f_{*}(t x), f_{*}(0)\right)=|t x|^{\alpha} d_{*}\left(f_{*}\left(\frac{x}{|x|}\right), f_{*}(0)\right) .
$$

On the other hand, for any $\beta \in(0,1)$ and $t$ small, there exists a constant $B$ so that

$$
d_{*}\left(f_{*}(t x), f_{*}(0)\right) \leq B|t x|^{\beta}
$$

by Lemma 8.6. Thus,

$$
d_{*}\left(f_{*}\left(\frac{x}{|x|}\right), f_{*}(0)\right) \leq B|t x|^{\beta-\alpha} .
$$

If $\alpha<1$, choose $\beta$ so that $\beta>\alpha$ and take the limit as $t \rightarrow 0$ to obtain

$$
d_{*}\left(f_{*}\left(\frac{x}{|x|}\right), f_{*}(0)\right)=0 .
$$

Since the choice of $x \in \mathbf{B}(1)$ is arbitrary, this contradicts the fact that $f_{*}$ is non-constant (cf. Lemma 6.1).

Using the fact that the order at a point on $D$ is $\geq 1$, we can prove Lipschitz continuity in $\mathbf{B}(1)$.

Theorem 8.2. Let $\mathbf{B}$ be a dimension-n, codimension-1 local model, g a Lipschitz Riemannian metric on $\mathbf{B}(r),(Y, d)$ an NPC space and $f:(\mathbf{B}(r), g) \rightarrow$ $(Y, d)$ a harmonic map. Then $f$ is Lipschitz in $\mathbf{B}(\varrho r)$ with Lipschitz constant depending on $\varrho \in(0,1),(\mathbf{B}(r), g)$ and the total energy $E^{f}$ of $f$.

Proof. For each $x \in \mathbf{B}(1)$, the normalized map $f \circ L_{x}$ (cf. Proposition 2.1) has order $\geq 1$ at 0 by Lemma 8.7. Thus, the order $\alpha_{x}$ of $f$ at $x$ is $\geq 1$. The result now follows from Theorem 4.1.

\subsection{At a higher codimension singular point}

Now we consider a dimension- $n$, codimension- $\nu$ local model where $\nu \geq 2$. Generally, we do not expect a harmonic map from this space to be Lipschitz continuous. On the other hand, we show that Lipschitz continuity can be proved with an additional assumption.

First, we establish some properties of the tangent map. Lemmas 8.8 to 8.10 below are the analogs of Lemmas 8.3, 8.4 and 7.4 corresponding to the codimension- $\nu$ case for $\nu \geq 2$. 
Lemma 8.8. Let $\mathbf{B}$ be a dimension-n, codimension- $\nu$ local model with $\nu \geq$ 2, $g$ a normalized Lipschitz metric defined on $\mathbf{B}(r)$ and $f:(\mathbf{B}(r), g) \rightarrow(Y, d)$ a harmonic map. Suppose $h_{k}:(\mathbf{B}(1), \delta) \rightarrow(Y, d), f_{k}:(\mathbf{B}, g) \rightarrow(Y, d), h_{*}=$ $f_{*}: \mathbf{B}(1) \rightarrow(Y, d)$ defined as in Section 6. Let $D_{\epsilon}$ be the $\epsilon$-neighborhood of $D$ and $D_{\epsilon}(r)=\mathbf{B}(r) \cap D_{\epsilon}$. Fix $R \in(0,1)$. For any $r \in(0, R)$, there exist $C$ and $\kappa>0, k_{0}$ sufficiently large and $\epsilon_{0}>0$ sufficiently small so that

$$
{ }^{\delta} E^{h_{k}}\left[D_{\epsilon}(r)\right] \leq C \epsilon^{\kappa}, \forall k>k_{0}, \epsilon<\epsilon_{0} .
$$

Proof. As in the proof of Lemma 8.3, there exists a constant $c_{3}$ so that

$$
\frac{\epsilon E_{x}^{h_{k}}(\epsilon)}{I_{x}^{h_{k}}(\epsilon)} \leq c_{3}
$$

Thus, by Theorem 4.2 ,

$$
E_{x}^{h_{k}}(\epsilon) \leq \frac{c_{3} I_{x}^{h_{k}}(\epsilon)}{\epsilon} \leq \frac{c_{3} C^{2} \epsilon^{2 \gamma+n-1}}{\epsilon}=: c_{4} \epsilon^{2 \gamma+n-2} .
$$

We can cover $D_{\epsilon}(r)$ be $\frac{c_{5}}{\epsilon^{n-\nu}}$ number of $(2 \epsilon)$-balls. Thus,

$$
E^{h_{k}}\left[D_{\epsilon}(r)\right] \leq \frac{c_{5}}{\epsilon^{n-\nu}} c_{4} \epsilon^{2 \gamma+n-2}=: c_{6} \epsilon^{2 \gamma-2+\nu} .
$$

The lower semicontinuity of energy implies that

$$
E^{f_{*}}\left[D_{\epsilon}(r)\right] \leq c_{6} \epsilon^{2 \gamma-2+\nu}
$$

Lemma 8.9. Let $h_{k}, f_{k}, h_{*}=f_{*}$ be as in Lemma 8.8. For $r \in(0,1)$,

$$
\lim _{k \rightarrow 0}{ }^{\delta} E^{h_{k}}(r)={ }^{\delta} E^{h_{*}}(r)
$$

and

$$
\lim _{k \rightarrow \infty}{ }^{\delta} E_{g_{k}}^{f_{k}}(r)={ }^{\delta} E^{f_{*}}(r) .
$$

Furthermore, the directional energies of $f_{k}, h_{k}$ converge to that of $f_{*}$. The maps $f_{*}$ is a homogeneous map of order $\alpha$, where $\alpha$ is the order of $f$ at 0 .

Proof. Using Lemma 8.8, we can follow the argument of the proof of Lemma 8.4. 
Lemma 8.10. Let $\mathbf{B}$ be a dimension-n, codimension- $\nu$ local model, $g$ a normalized Lipschitz metric defined on $\mathbf{B}(r),(Y, d)$ an NPC space, $f:(\mathbf{B}(r), g)$ $\rightarrow(Y, d)$ a harmonic map and $f_{*}: \mathbf{B}(1) \rightarrow Y_{*}$ its tangent map. Then for $x, y$ on the same wedge at a distance $\rho$ away from $D(1)$, we have

$$
d\left(f_{*}(x), f_{*}(y)\right) \leq L|x-y|
$$

where $L$ is depending only on $r, \mathbf{B}(1)$ and ${ }^{\delta} E^{h}$.

Proof. If $x, y \in \mathbf{B}(1 / 2)$ are two points on the same wedge at a distance $\rho$ away from $D(1)$, then Lemma 7.4 implies

$$
d\left(h_{k}(x), h_{k}(y)\right) \leq L|x-y|
$$

where $c$ is depending on $r$ and ${ }^{\delta} E^{h_{k}}$. Thus, the result follows from the uniform convergence of $h_{k}$ to $f_{*}$ and the convergence of the energy of $h_{k}$ to that of $f_{*}$.

Lemma 8.11. Let $\mathbf{B}$ be a dimension-n, codimension- $\nu$ local model, $g$ a normalized Lipschitz metric defined on $\mathbf{B}(r)$ and $f:(\mathbf{B}(r), g) \rightarrow(Y, d)$ a harmonic map. Then its tangent map $f_{*}: \mathbf{B}(1) \rightarrow Y_{*}$ is homogeneous of order $\alpha$ where $\alpha$ is the order of $f$ at 0 .

Proof. Follows immediately from Lemmas 6.3 and 8.9.

Our next goal is to relate the order (and hence the Hölder exponent) of a harmonic map to the first eigenvalue associated with the domain and the target space. We start with a general definition of the first eigenvalue. Let $G$ be a Riemannian complex with volume form $d s$ and $T$ an NPC space. The center of mass of a map $\varphi \in L^{2}(G, T)$ is a point $\bar{\varphi} \in T$ so that

$$
\int_{G} d_{T}^{2}(\varphi, \bar{\varphi}) d s=\inf _{P \in Y} \int_{G} d_{T}^{2}(\varphi, P) d s .
$$

The unique existence of such a point is guaranteed by the NPC condition (cf. [11, Proposition 2.5.4]). Now let $\mathcal{G}(T)$ be the set of Lipschitz maps $\varphi: G \rightarrow T$ into an NPC space $T$ and define the first eigenvalue of $G$ with values in $T$ as

$$
\lambda_{1}(G, T)=\inf _{\mathcal{G}(T)} \frac{\int_{G}|\nabla \varphi|^{2} d s}{\int_{G} d_{T}^{2}(\varphi, \bar{\varphi}) d s} .
$$

In the applications, $G$ will be a spherical complex associated with the domain of the map and the NPC space $T$ will be a tangent cone of the target NPC 
space $Y$. The following results appear in [6] in the case when the domain is of dimension 2 .

Lemma 8.12. Suppose $f: \mathbf{B}(r) \rightarrow(Y, d)$ is a bounded map, $\sigma \in(0, r)$ and $Q \in Y$ so that

$$
\int_{\partial \mathbf{B}(\sigma)} d^{2}(f, Q) d \Sigma=\inf _{P \in Y} \int_{\partial \mathbf{B}(\sigma)} d^{2}(f, P) d \Sigma .
$$

If $\pi: Y \rightarrow T_{Q} Y$ is the projection map into the tangent cone of $Y$ at $Q$, then

$$
\int_{\partial \mathbf{B}(\sigma)} d_{T_{Q} Y}^{2}(\pi \circ f, 0) d \Sigma=\inf _{V \in T_{Q} Y} \int_{\partial \mathbf{B}(\sigma)} d_{T_{Q} Y}^{2}(\pi \circ f, V) d \Sigma,
$$

where 0 is the origin of $T_{Q} Y$.

Proof. Let $t \mapsto c(t)$ be a geodesic so that $c(0)=Q$. By the minimizing property of $c(0)=Q$, we have

$$
0 \leq \int_{\partial \mathbf{B}(\sigma)} d^{2}(f, c(t)) d \Sigma-\int_{\partial \mathbf{B}(\sigma)} d^{2}(f, c(0)) d \Sigma .
$$

Furthermore, by Bridson and Haefliger [1] Corollary II 3.6, we have

$$
\lim _{t \rightarrow 0} \frac{d(f(y), c(t))-d(f(y), c(0))}{t}=-\cos \angle\left(c, \gamma_{y}\right)
$$

where $\gamma_{y}$ is the geodesic from $c(0)$ to $f(y)$ and $\angle\left(\gamma_{y}, c\right)$ is the angle between $\gamma_{y}$ and $c$ at $c(0)=Q$. Therefore,

$$
\begin{aligned}
0 & \leq \lim _{t \rightarrow 0} \int_{\partial \mathbf{B}(\sigma)} \frac{d^{2}(f, c(t))-d^{2}(f, c(0))}{t} d \Sigma \\
& =\lim _{t \rightarrow 0} \int_{\partial \mathbf{B}(\sigma)} \frac{d(f, c(t))-d(f, c(0))}{t}(d(f, c(t))+d(f, c(0))) d \Sigma \\
& =-2 \int_{\partial \mathbf{B}(\sigma)} \cos \angle\left(\gamma_{y}, c\right) d(f, c(0)) d \Sigma .
\end{aligned}
$$

Let $[c]$ be the equivalence class of $c$ and $V=([c], 1) \in T_{Q} Y$. Since $\pi \circ \gamma_{y}$ is the (radial) geodesic from the origin 0 to $\pi \circ f(y)$ in $T_{Q} Y$,

$$
\cos \angle\left(\gamma_{y}, c\right) d(f(y), f(0))=<\pi \circ f(y), V>
$$


and thus

$$
0 \leq-\int_{y \in \partial \mathbf{B}(\sigma)}<\pi \circ f(y), V>d \Sigma
$$

By the continuity of the inner product, (8.4) holds for all $V=\left(V_{0}, t\right) \in T_{Q} Y$ where $V_{0}=V /|V|$. Therefore, for $t \geq 0$,

$$
\begin{aligned}
& \int_{\partial \mathbf{B}(\sigma)} d_{T_{Q} Y}^{2}\left(\pi \circ f(y),\left(V_{0}, t\right)\right) d \Sigma \\
& \quad=\int_{\partial \mathbf{B}(\sigma)} t^{2}+|\pi \circ f(y)|^{2}-2 t<\pi \circ f(y), V_{0}>d \Sigma \\
& \quad \geq \int_{\partial \mathbf{B}(\sigma)}|\pi \circ f(y)|^{2} d \Sigma \\
& =\int_{\partial \mathbf{B}(\sigma)} d_{T_{Q} Y}^{2}(\pi \circ f(y), 0) d \Sigma .
\end{aligned}
$$

Corollary 8.1. Suppose $f: \mathbf{B}(r) \rightarrow(Y, d)$ is a bounded map, $\sigma \in(0, r)$ and $Q \in Y$ so that

$$
\int_{\partial \mathbf{B}(\sigma)} d^{2}(f, Q) d \Sigma=\inf _{P \in Y} \int_{\partial \mathbf{B}(\sigma)} d^{2}(f, P) d \Sigma .
$$

If $\pi: Y \rightarrow T_{Q} Y$ is the projection map into the tangent cone of $Y$ at $Q$ and $\sigma: \mathbf{B}(1) \rightarrow \mathbf{B}(\sigma)$ is defined by $\sigma(x)=\sigma x$, then

$$
\frac{\int_{\partial \mathbf{B}(1)}\left|\nabla^{\partial}(\pi \circ f \circ \sigma)(x)\right|^{2} d \Sigma}{\int_{\partial \mathbf{B}(1)}|\pi \circ f \circ \sigma(x)|^{2} d \Sigma} \geq \lambda_{1}\left(\partial \mathbf{B}(1), T_{Q} Y\right),
$$

where $\nabla^{\partial}$ indicates that we are taking the tangential part of the energy density function on $\partial \mathbf{B}(1)$.

Proof. By Lemma 8.12, the center of mass of the map $\pi \circ f \circ \sigma$ is 0 . Thus, the assertion follows immediately from the definition of $\lambda_{1}\left(\mathbf{B}(1), T_{Q} Y\right)$.

A consequence of Corollary 8.1 is the following theorem which associates the first eigenvalue with the order of a harmonic map.

Theorem 8.3. Let $\mathbf{B}$ be a dimension-n, codimension- $\nu$ local model, $g$ a normalized Lipschitz Riemannian metric defined on $\mathbf{B}(r),(Y, d)$ an $N P C$ 
space and $f:(\mathbf{B}(r), g) \rightarrow(Y, d)$ be a harmonic map. If $\lambda_{1}\left(\partial \mathbf{B}(1), T_{Q} Y\right) \geq \beta$ (> $\beta$ ) for all $Q \in Y$ then $\alpha(\alpha+n-2) \geq \beta(>\beta)$, where $\alpha$ is the order of $f$ at 0 .

Proof. By inequalities (6.2), (6.3) and (6.6), it suffices to assume that the volume form and the directional derivatives are with respect to the Euclidean metric $\delta$. This assumption is clearly without any loss of generality since in this proof we are interested in rescalings of $\mathbf{B}(\sigma)$ to unit size as $\sigma \rightarrow 0$ and at small scales the metric $g$ is approximately Euclidean. We emphasize that $f$ is harmonic with respect to the metric $g$ which is not necessarily Euclidean.

Let $\sigma_{i} \rightarrow 0$ so that $f_{\sigma_{i}} \rightarrow f_{*}: \mathbf{B}(1) \rightarrow Y_{*}$. From Lemma 8.9, there exists $\lambda$ so that

$$
\lim _{\sigma_{i} \rightarrow 0} \int_{\partial \mathbf{B}(\lambda)}\left|\nabla^{\partial} f_{\sigma_{i}}\right|^{2} d \Sigma=\int_{\partial \mathbf{B}(\lambda)}\left|\nabla^{\partial} f_{*}\right| d \Sigma .
$$

By Gromov and Schoen [9, pp. 200-201], we have

$$
\lim _{\sigma \rightarrow 0} \frac{\sigma \lambda E(\sigma \lambda)}{\int_{\partial \mathbf{B}(\sigma \lambda)} d^{2}\left(f, Q_{\sigma \lambda}\right) d \Sigma}=\lim _{\sigma \rightarrow 0} \frac{\sigma \lambda E(\sigma \lambda)}{\int_{\partial \mathbf{B}(\sigma \lambda)} d^{2}(f, f(0)) d \Sigma},
$$

where $Q_{\sigma} \in Y$ is the point so that

$$
\int_{\partial \mathbf{B}(\sigma)} d^{2}\left(f, Q_{\sigma}\right) d \Sigma=\inf _{Q \in Y} \int_{\partial \mathbf{B}(\sigma)} d^{2}(f, Q) d \Sigma .
$$

This then implies

$$
\lim _{\sigma \rightarrow 0} \frac{\int_{\partial \mathbf{B}(\sigma \lambda)} d^{2}\left(f, Q_{\sigma \lambda}\right) d \Sigma}{\int_{\partial \mathbf{B}(\sigma \lambda)} d^{2}(f, f(0)) d \Sigma}=1 .
$$

Let $Q_{i}:=Q_{\sigma_{i}} \in Y$ and $\pi_{i}: Y \rightarrow T_{Q_{i}} Y$ be a projection map into the tangent cone of $Y$ at $Q_{i}$. By Lemma 8.12,

$$
\int_{\partial \mathbf{B}\left(\sigma_{i} \lambda\right)} d^{2}\left(\pi_{i} \circ f, 0\right) d \Sigma=\inf _{V \in T_{Q_{i}} Y} \int_{\partial \mathbf{B}\left(\lambda \sigma_{i}\right)} d^{2}\left(\pi_{i} \circ f, V\right) d \Sigma .
$$

Additionally,

$$
d^{2}\left(f, Q_{i}\right)=\left|\pi_{i} \circ f\right|^{2} \text { and }\left|\nabla^{\partial} f\right|^{2} \geq\left|\nabla^{\partial}\left(\pi_{i} \circ f\right)\right|^{2}
$$


since $\pi_{i}$ is distance non-increasing. Thus, by (8.5) and (8.7),

$$
\begin{aligned}
\lim _{\sigma_{i} \rightarrow 0} \frac{\lambda^{2} \int_{\partial \mathbf{B}(\lambda)}\left|\nabla^{\partial} f_{\sigma_{i}}\right|^{2} d \Sigma}{\int_{\partial \mathbf{B}(\lambda)} d_{\sigma_{i}}^{2}\left(f_{\sigma_{i}}, f_{\sigma_{i}}(0)\right) d \Sigma} & =\lim _{\sigma_{i} \rightarrow 0} \frac{\left(\sigma_{i} \lambda\right)^{2} \int_{\partial \mathbf{B}\left(\sigma_{i} \lambda\right)}\left|\nabla^{\partial} f\right|^{2} d \Sigma}{\int_{\partial \mathbf{B}\left(\sigma_{i} \lambda\right)} d^{2}(f, f(0)) d \Sigma} \\
& =\lim _{\sigma_{i} \rightarrow 0} \frac{\left(\sigma_{i} \lambda\right)^{2} \int_{\partial \mathbf{B}\left(\sigma_{i} \lambda\right)}\left|\nabla^{\partial} f\right|^{2} d \Sigma}{\int_{\partial \mathbf{B}\left(\sigma_{i} \lambda\right)} d^{2}\left(f, Q_{i}\right) d \Sigma} \\
& \geq \lim _{\sigma_{i} \rightarrow 0} \frac{\left(\sigma_{i} \lambda\right)^{2} \int_{\partial \mathbf{B}\left(\sigma_{i} \lambda\right)}\left|\nabla^{\partial}\left(\pi_{i} \circ f\right)\right|^{2} d \Sigma}{\int_{\partial \mathbf{B}\left(\sigma_{i} \lambda\right)}\left|\pi_{i} \circ f\right|^{2} d \Sigma}
\end{aligned}
$$

By change of coordinates $y=\sigma_{i} \lambda x,(8.6)$ and Corollary 8.1,

$$
\begin{aligned}
\frac{\left(\sigma_{i} \lambda\right)^{2} \int_{y \in \partial \mathbf{B}\left(\sigma_{i} \lambda\right)}\left|\nabla^{\partial}\left(\pi_{i} \circ f\right)(y)\right|^{2} d \Sigma}{\int_{\partial \mathbf{B}\left(\sigma_{i} \lambda\right)}\left|\pi_{i} \circ f(y)\right|^{2} d \Sigma} & =\frac{\int_{x \in \partial \mathbf{B}(1)}\left|\nabla^{\partial}\left(\pi_{i} \circ f \circ\left(\sigma_{i} \lambda\right)\right)(x)\right|^{2} d \Sigma}{\int_{x \in \partial \mathbf{B}(1)}\left|\left(\pi_{i} \circ f \circ\left(\sigma_{i} \lambda\right)\right)(x)\right|^{2} d \Sigma} \\
& =\frac{\int_{x \in \mathbf{B}(1)} \mid \nabla^{\partial}\left(\left.\pi_{i} \circ f \circ\left(\sigma_{i} \lambda\right)\right|^{2}(x) d \Sigma\right.}{\int_{x \in \mathbf{B}(1)}\left|\left(\pi_{i} \circ f \circ\left(\sigma_{i} \lambda\right)\right)(x)\right|^{2} d \Sigma} \\
& \geq \lambda_{1}\left(\partial \mathbf{B}(1), T_{Q_{i}} Y\right) \\
& \geq \beta(>\beta) .
\end{aligned}
$$

Therefore,

$$
\begin{aligned}
R & :=\frac{\int_{\partial \mathbf{B}(1)}\left|\nabla^{\partial} f_{*}\right|^{2} d \Sigma}{\int_{\partial \mathbf{B}(1)} d^{2}\left(f_{*}, f_{*}(0)\right) d \Sigma} \\
& =\frac{\lambda^{2} \int_{\partial \mathbf{B}(\lambda)}\left|\nabla^{\partial} f_{*}\right|^{2} d \Sigma}{\int_{\partial \mathbf{B}(\lambda)} d^{2}\left(f_{*}, f_{*}(0)\right) d \Sigma} \\
& =\lim _{\sigma_{i} \rightarrow 0} \frac{\lambda^{2} \int_{\partial \mathbf{B}(\lambda)}\left|\nabla^{\partial} f_{\sigma_{i}}\right|^{2} d \Sigma}{\int_{\partial(\lambda)} d_{\sigma_{i}}^{2}\left(f_{\sigma_{i}}, f_{\sigma_{i}}(0)\right) d \Sigma} \\
& \geq \beta(>\beta) .
\end{aligned}
$$

For $y \in \partial \mathbf{B}(1)$, the homogeneity of $f_{*}$ implies

$$
d\left(f_{*}(r y), f_{*}(0)\right)=r^{\alpha} d\left(f_{*}(y), f_{*}(0)\right)
$$


and hence

$$
\begin{aligned}
E^{f_{*}}(1)= & \int_{y \in \partial \mathbf{B}(1)} \int_{0}^{1}\left(\left|\frac{\partial f_{*}}{\partial r}(r y)\right|^{2}+\frac{1}{r^{2}}\left|\nabla^{\partial} f_{*}(r y)\right|^{2}\right) r^{n-1} d r d \Sigma \\
= & \int_{y \in \partial \mathbf{B}(1)} \int_{0}^{1}\left(\alpha^{2} r^{2 \alpha+n-3} d^{2}\left(f_{*}(y), f_{*}(0)\right)\right. \\
& \left.+r^{2 \alpha+n-3}\left|\nabla^{\partial} f_{*}(y)\right|^{2}\right) d r d \Sigma \\
= & \frac{\alpha^{2}}{2 \alpha+n-2} \int_{y \in \partial \mathbf{B}(1)} d^{2}\left(f_{*}(y), f_{*}(0)\right) d \Sigma \\
& +\frac{1}{2 \alpha+n-2} \int_{y \in \partial \mathbf{B}(1)}\left|\nabla^{\partial} f_{*}(y)\right|^{2} d \Sigma
\end{aligned}
$$

Thus,

$$
\alpha=\frac{E^{f_{*}}(1)}{I^{f_{*}}(1)}=\frac{\alpha^{2}}{2 \alpha+n-2}+\frac{1}{2 \alpha+n-2} R
$$

and

$$
\alpha(\alpha+n-2)=R \geq \beta(>\beta) .
$$

Given $\mathbf{B}$ and any $x \in \mathbf{B}(r)$, consider $f \circ L_{x}: \mathbf{B}_{x}^{\prime}(r(x)) \rightarrow(Y, d)$ of Proposition 2.1 where $\mathbf{B}_{x}^{\prime}$ is a local model associated with the point $x$. We let

$$
\lambda_{1}:=\inf _{x \in \mathbf{B}(r), Q \in Y} \lambda_{1}\left(\partial \mathbf{B}_{x}^{\prime}(1), T_{Q} Y\right)
$$

Corollary 8.2. Let $\mathbf{B}$ be a dimension-n, codimension- $\nu$ local model, $g$ a normalized Lipschitz Riemannian metric defined on $\mathbf{B}(r)$ and $(Y, d)$ an NPC space. If $\lambda_{1} \geq \alpha(\alpha+n-2)$ and $f:(\mathbf{B}(r), g) \rightarrow(Y, d)$ is a harmonic map, then $f$ is Hölder continuous with Hölder exponent $\alpha$ in $\mathbf{B}(\varrho r)$ for $\varrho \in(0,1)$.

Proof. For any $x \in \mathbf{B}(\varrho r)$, Theorem 8.3 says that the assumption $\lambda_{1} \geq \alpha(\alpha+$ $n-2$ ) for all $Q \in Y$ implies the order of $f$ at $x$ is $\geq \alpha$. The result now follows from Theorem 4.1.

We now give a sufficient condition implying that the order of a harmonic map is $\geq 1$. For each $x \in D$, let $N(x)$ be the $\nu$-plane perpendicular to $D$ at $x$. Note that $|x|<1$ implies that $\partial \mathbf{B}(1) \cap N(x)$ is a spherical $(\nu-1)$-complex. We first need the following lemma. 
Lemma 8.13. Let $f_{*}: \mathbf{B}(1) \rightarrow Y_{*}$ be a tangent map of a harmonic map. If the order of $f_{*}$ is not equal to 1 , then $f_{*}$ is constant in the direction parallel to $D$.

Proof. By Lemma 6.4, we may assume that $f_{*}$ maps into a cone with $f_{*}(0)$ equal to the vertex. Also, we may assume by homogeneity of $f_{*}$ that the domain of $f_{*}$ is $\mathbf{B}$. Let $x, y \in \mathbf{B}(1)$ be points on the same wedge and same distance to $D$ and $x^{\prime}=t x, y^{\prime}=t y$. Then

$$
t^{\alpha} d_{*}\left(f_{*}(x), f_{*}(y)\right)=d_{*}\left(f_{*}\left(x^{\prime}\right), f_{*}\left(y^{\prime}\right)\right) \leq L\left|x^{\prime}-y^{\prime}\right|=C t,
$$

where $L$ is the Lipschitz constant of $f_{*}$ and $C$ is a constant depending on $L$ and on the angle between the line from $x$ to 0 and $y$ to 0 , respectively. Thus,

$$
d_{*}\left(f_{*}(x), f_{*}(y)\right) \leq C t^{1-\alpha}
$$

and the lemma follows by letting $t \rightarrow 0$ if $\alpha<1$ or $t \rightarrow \infty$ if $\alpha>1$.

Theorem 8.4. Let $\mathbf{B}$ be a dimension-n, codimension- $\nu$ local model, $g$ a normalized Lipschitz Riemannian metric defined on $\mathbf{B}(r),(Y, d)$ an NPC space and $f:(\mathbf{B}(r), g) \rightarrow(Y, d)$ be a harmonic map. If $\lambda_{1}(\partial \mathbf{B}(1) \cap N(0)$, $\left.T_{Q} Y\right) \geq \beta(>\beta)$ and $\alpha<1$ for all $Q \in Y$, then the order $\alpha$ of $f$ at 0 satisfies $\alpha(\alpha+\nu-2) \geq \beta(>\beta)$.

Proof. By the homogeneity of $f_{*}$,

$$
\begin{aligned}
\alpha & =\frac{E^{f_{*}}(1)}{I^{f_{*}(1)}} \\
& =\frac{\int_{\mathbf{B}(1)}\left|\nabla f_{*}\right|^{2} d \mu}{\int_{\partial \mathbf{B}(1)} d^{2}\left(f_{*}, 0\right) d \Sigma} \\
& =\frac{\int_{x \in D} \int_{y \in \mathbf{B}(1) \cap N(x)}\left|\nabla f_{*}\right|^{2} d y d x}{\int_{x \in D} \frac{1}{\left(1-|x|^{2}\right)^{1 / 2}} \int_{\partial \mathbf{B}(1) \cap N(x)} d^{2}\left(f_{*}, 0\right) d \Sigma d x} .
\end{aligned}
$$

We use the notation $\nabla^{N}$ to indicate the we are taking the directional energy of $f_{*}$ on $\partial \mathbf{B}(1) \cap N(x)$. Using Lemma 8.13, $\left|\nabla f_{*}\right|^{2}=\left|\nabla^{N} f_{*}\right|^{2}$ and hence 


$$
\begin{aligned}
\int_{y \in \mathbf{B}(1) \cap N(x)}\left|\nabla f_{*}\right|^{2}(y) d y \\
=\int_{y \in \mathbf{B}(1) \cap N(x)}\left|\nabla^{N} f_{*}\right|^{2}(y) d y \\
=\int_{y \in \mathbf{B}\left(\left(1-|x|^{2}\right)^{1 / 2}\right) \cap N(0)}\left|\nabla^{N} f_{*}\right|^{2}(y) d y \\
=\left(1-|x|^{2}\right)^{\alpha-1} \int_{y \in \mathbf{B}\left(\left(1-|x|^{2}\right)^{1 / 2}\right) \cap N(0)}\left|\nabla^{N} f_{*}\right|^{2}\left(\left(1-|x|^{2}\right)^{-1 / 2} y\right) d y
\end{aligned}
$$

Here, the second equality follows from translation in direction parallel to $D$ and the last equality follows from the homogeneity of $f_{*}$.

Now apply the change of coordinates $z=\left(1-|x|^{2}\right)^{-1 / 2} y$ to obtain

$$
\begin{gathered}
\int_{y \in \mathbf{B}\left(\left(1-|x|^{2}\right)^{1 / 2}\right) \cap N(0)}\left|\nabla^{N} f_{*}\right|^{2}\left(\left(1-|x|^{2}\right)^{-1 / 2} y\right) d y \\
=\left(1-|x|^{2}\right)^{\frac{\nu}{2}} \int_{\mathbf{B}(1) \cap N(0)}\left|\nabla^{N} f_{*}\right|^{2}(z) d z .
\end{gathered}
$$

Hence the numerator in $(8.8)$ is

$$
\int_{x \in D}\left(1-|x|^{2}\right)^{\frac{\nu-2+2 \alpha}{2}} d x \int_{\mathbf{B}(1) \cap N(0)}\left|\nabla^{N} f_{*}\right|^{2}(z) d z .
$$

Similarly, the denominator of (8.8) is

$$
\begin{aligned}
\int_{x \in D} & \frac{1}{\left(1-|x|^{2}\right)^{1 / 2}} d x \int_{\partial \mathbf{B}(1) \cap N(x)} d^{2}\left(f_{*}, 0\right) d \Sigma \\
= & \int_{x \in D}\left(1-|x|^{2}\right)^{\frac{\nu-2+2 \alpha}{2}} d x \int_{\partial \mathbf{B}(1) \cap N(0)} d^{2}\left(f_{*}, 0\right) d \Sigma .
\end{aligned}
$$

Thus, as in the proof of Theorem 8.3, we obtain

$$
\begin{aligned}
\alpha & =\frac{\int_{\mathbf{B}(1) \cap N(0)}\left|\nabla^{N} f_{*}\right|^{2} d y}{\int_{\partial \mathbf{B}(1) \cap N(0)} d^{2}\left(f_{*}, 0\right) d \Sigma} \\
& =\frac{\alpha^{2}}{2 \alpha+\nu-2}+\frac{R}{2 \alpha+\nu-2}
\end{aligned}
$$

and hence

$$
\alpha(\alpha+\nu-2)=R \geq \beta(>\beta) .
$$


Given $\mathbf{B}$ and any $x \in \mathbf{B}(r)$, consider $f \circ L_{x}: \mathbf{B}_{x}^{\prime}(r(x)) \rightarrow(Y, d)$ of Proposition 2.1 where $\mathbf{B}_{x}^{\prime}$ is a local model associated with the point $x$. We let

$$
\lambda_{1}^{N}:=\inf _{x \in \mathbf{B}(r), Q \in Y} \lambda_{1}\left(\partial \mathbf{B}_{x}^{\prime}(1) \cap N(0), T_{Q} Y\right) .
$$

Corollary 8.3. Let $\mathbf{B}$ be a dimension-n, codimension- $\nu$ local model, $g$ a Lipschitz Riemannian metric defined on $\mathbf{B}(r)$ and $(Y, d)$ an NPC space. If $\lambda_{1}^{N} \geq \nu-1$ and $f: \mathbf{B}(r) \rightarrow(Y, d)$ is a harmonic map, then $f$ is Lipschitz continuous in $\mathbf{B}(\varrho r)$ for $\varrho \in(0,1)$.

Proof. For any $x \in \mathbf{B}(\varrho r)$, Theorem 8.4 says that the assumption $\lambda_{1}^{N} \geq$ $\nu-1$ implies that the order of $f$ at $x$ is $\geq 1$. The result now follows from Theorem 4.1.

\section{Main Theorem}

Here, we collect the regularity results from the previous sections to summarize our main regularity theorem for Lipschitz Riemannian complexes.

Theorem 9.1. Let $B(r)$ be a ball of radius $r$ around a point $x$ in an admissible complex $X$ endowed with a Lipschitz Riemannian metric $g,(Y, d)$ an $N P C$ space and $f:(B(r), g) \rightarrow(Y, d)$ a harmonic map.

(1) If $x \in X-X^{(n-2)}$, let $d$ denote the distance of $x$ to $X^{(n-2)}$. Then for $\varrho \in(0,1)$ and $d^{\prime} \leq \min \{\varrho r, \varrho d\}, f$ is Lipschitz continuous in $B\left(d^{\prime}\right)$ with Lipschitz constant depending on the total energy of the map $f$, $(B(r), g), d$ and $\varrho$.

(2) If $x \in X^{(k)}-X^{(k-1)}$ for $k=0, \ldots, n-2$, let $d$ denote the distance of $x$ to $X^{(k-1)}$. Then for $\varrho \in(0,1)$ and $d^{\prime} \leq \min \{\varrho r, \varrho d\}, f$ is Hölder continuous in $B\left(d^{\prime}\right)$ with Hölder exponent and constant depending on the total energy of the map $f,(B(r), g), d$ and $\varrho$. More precisely, the Hölder exponent $\alpha$ has a lower bound given by the following: If $\lambda_{1}^{N} \geq$ $\beta(>\beta)$ then $\alpha(\alpha+n-k-2) \geq \beta(>\beta)$. In particular, if $\lambda_{1}^{N} \geq n-$ $k-1$, then $f$ is Lipschitz continuous in a neighborhood of $x$.

Proof. The assertion follows from Theorems 8.2, 8.4 and Corollary 8.3.

\section{Acknowledgment}

G.D. supported by research grant NSF DMS-0604930 and C.M. supported by research grant NSF DMS-0450083. 


\section{References}

[1] M. Bridson and A. Haefliger, Metric spaces of non-positive curvature, Springer, Berlin, 1991.

[2] J. Chen, On energy minimizing mappings between and into singular spaces, Duke Math. J. 79 (1995), 77-99.

[3] K. Corlette. Archemedean superrigidity and hyperbolic geometry, Ann. of Math. 135 (1992), 165-182.

[4] G. Daskalopoulos and C. Mese, Harmonic maps from a 2-complex, Commu. Anal. Geom. 14 (2006), 1-52.

[5] G. Daskalopoulos and C. Mese, Harmonic maps from a simplicial complex and geometric rigidity, J. Differential Geom. 78 (2008), 269-293.

[6] G. Daskalopoulos and C. Mese, Fixed point and rigidity theorems for harmonic maps from simplicial complexes into NPC spaces, Geom. Dedicata (2009), $14133-57$.

[7] J. Eells and B. Fuglede, Harmonic maps between Riemannian polyhedra, Cambridge Tracts Maths., 142, Cambridge University Press, Cambridge 2001.

[8] B. Fuglede, Hölder continuity of harmonic maps from Riemannian polyhedra to spaces of upper bounded curvature, Calc. Var. Partial Differential Equations 16(4) (2003), 375-403.

[9] M. Gromov and R. Schoen, Harmonic maps into singular spaces and $p$ adic superrigidity for lattices in groups of rank one, Publ. Math. IHES 76 (1992), 165-246.

[10] J. Jost, Nonpositive curvature: geometric and analytic aspects, Lect. in Maths, ETH Zürich, Birkhäuser Verlag, 1997, MR1451625, Zbl 0896.53002.

[11] N. Korevaar and R. Schoen, Sobolev spaces and harmonic maps for metric space targets, Comm. Anal. Geom. 1 (1993), 561-659.

[12] N. Korevaar and R. Schoen, Global existence theorem for harmonic maps to non-locally compact spaces, Comm. Anal. Geom. 5 (1997), $333-387$. 
[13] C. Mese, Regularity of harmonic maps from a flat complex, Progress in Non-Linear Differential Equations, Birkhauser-Verlag, Basel, 2004, 133-148.

[14] C. Mese, Harmonic maps into spaces with an upper curvature bound in the sense of Alexandrov, Math. Z. 242 (2002), 633-661.

[15] T. Serbinowski, Boundary regularity of harmonic maps to nonpositively curved metric spaces, Comm. Anal. Geom. 2 (1994), 139-154.

[16] L. Simon, Theorems on regularity and singularity of energy minimizing maps, Birkhäuser, Basel, 1996.

Department of Mathematics

BROWN UNIVERSITY

Providence, RI 02912, USA

E-mail address: daskal@math.brown.edu

Department of Mathematics

JOHNS HOPKINS UNIVERSITY

3400 N. Charles Street

BALTimore, MD 21218, USA

E-mail address: cmese@math.jhu.edu

RECEIVED April 2, 2009 
